\title{
The Decomposition of Global Conformal Invariants: Some Technical Proofs. I
}

\author{
Spyros ALEXAKIS
}

Department of Mathematics, University of Toronto, 40 St. George Street, Toronto, Canada E-mail: alexakis@math.toronto.edu

Received April 01, 2010, in final form February 15, 2011; Published online February 26, 2011 doi:10.3842/SIGMA.2011.019

\begin{abstract}
This paper forms part of a larger work where we prove a conjecture of Deser and Schwimmer regarding the algebraic structure of "global conformal invariants"; these are defined to be conformally invariant integrals of geometric scalars. The conjecture asserts that the integrand of any such integral can be expressed as a linear combination of a local conformal invariant, a divergence and of the Chern-Gauss-Bonnet integrand.
\end{abstract}

Key words: conormal geometry; renormalized volume; global invariants; Deser-Schwimmer conjecture

2010 Mathematics Subject Classification: 53B20; 53A55

\section{Introduction}

The present paper complements the monograph [3] and also $[1,2,4,5]$ in proving a conjecture of Deser and Schwimmer [15] regarding the algebraic structure of "global conformal invariants" 1 . Here we provide a proof of certain lemmas which were announced in the second chapter of [3]. The proofs of these claims do not logically depend on material appearing elsewhere in this entire work.

For the reader's convenience, we briefly review the Deser-Schwimmer conjecture.

We recall that a global conformal invariant is an integral of a natural scalar-valued function of Riemannian metrics, $\int_{M^{n}} P(g) d V_{g}$, which remains invariant under conformal re-scalings of the underlying metric ${ }^{2}$. More precisely, $P(g)$ is assumed to be a linear combination, $P(g)=$ $\sum_{l \in L} a_{l} C^{l}(g)$, where each $C^{l}(g)$ is a complete contraction in the form:

$$
\operatorname{contr}^{l}\left(\nabla^{\left(m_{1}\right)} R \otimes \cdots \otimes \nabla^{\left(m_{s}\right)} R\right) .
$$

Here each factor $\nabla^{(m)} R$ stands for the $m^{\text {th }}$ iterated covariant derivative of the curvature tensor $R . \nabla$ is the Levi-Civita connection of the metric $g$ and $R$ is the curvature associated to this connection. The contractions are taken with respect to the quadratic form $g^{i j}$. In the present paper, along with $[3,5]$ we prove:

Theorem 1.1. Assume that $P(g)=\sum_{l \in L} a_{l} C^{l}(g)$, where each $C^{l}(g)$ is a complete contraction in the form (1.1), with weight $-n$. Assume that for every closed Riemannian manifold $\left(M^{n}, g\right)$ and every $\phi \in C^{\infty}\left(M^{n}\right)$ :

$$
\int_{M^{n}} P\left(e^{2 \phi} g\right) d V_{e^{2 \phi} g}=\int_{M^{n}} P(g) d V_{g}
$$

\footnotetext{
${ }^{1} \mathrm{~A}$ formulation of the conjecture which is closer to the mathematical terminology used here can also be found in [23].

${ }^{2}$ See the introduction of [3] for a detailed discussion on scalar Riemannian invariants and on applications of the Deser-Schwimmer conjecture.
} 
We claim that $P(g)$ can then be expressed in the form:

$$
P(g)=W(g)+\operatorname{div}_{i} T^{i}(g)+(\text { const }) \cdot \operatorname{Pfaff}\left(R_{i j k l}\right) .
$$

Here $W(g)$ stands for a local conformal invariant of weight $-n$ (meaning that $W\left(e^{2 \phi} g\right)=$ $e^{-n \phi} W(g)$ for every $\left.\phi \in C^{\infty}\left(M^{n}\right)\right), \operatorname{div}_{i} T^{i}(g)$ is the divergence of a Riemannian vector field of weight $-n+1, \operatorname{Pfaff}\left(R_{i j k l}\right)$ is the Pfaffian of the curvature tensor, and (const) is a constant.

Remark. The notion of a "global conformal invariant" is closely related to the algebraic structure of conformal anomalies ${ }^{3}$, which were the motivation of Deser and Schwimmer [15] (see also especially [23]); however the two notions are not identical: very broadly speaking, a conformal anomaly represents the failure of an action that depends on a metric $g$ to be conformally invariant. A well-studied such anomaly (which directly gives rise to the global conformal invariants we study here) is considered in [23] in the context of the AdS-CFT correspondence; it is the renormalized volume $\mathcal{A}$ of a Poincaré-Einstein metric $\left(X^{n+1}, h\right)$ with conformal infinity $\left(M^{n},[g]\right)$, when $n$ is even. In that setting if $\dot{g}=2 \phi g$, then $\dot{\mathcal{A}}(g)=\int_{M^{n}} P(g) \phi d V_{g}$. Furthermore as noted by Henningson-Skenderis [23], the integral $\int_{M^{n}} P(g) d V_{g}$ is a global conformal invariant in the sense described above. (This fact was noted in [23] on general grounds; Graham in [21] showed the conformal invariance of $\int_{M^{n}} P(g) d V_{g}$ by explicitly studying the conformal variation of the renormalized volume.) Thus, the decomposition (1.2) that we derive for $P(g)$ corresponds to a decomposition of the integrand in the conformal anomaly; this conforms with the stipulation of $[23]^{4}$.

We now digress in order to discuss the relation of this entire work (consisting of the present paper and $[3,4,5])$ with classical and recent work on local invariants in various geometries.

Local invariants and Fefferman's program for the Bergman and Szegö kernels. The theory of local invariants of Riemannian structures (and indeed, of more general geometries, e.g. conformal, projective, or CR) has a long history. The original foundations of this field were laid in the work of Hermann Weyl and Élie Cartan, see [26, 14]. The task of writing out local invariants of a given geometry is intimately connected with understanding which polynomials in a space of tensors with given symmetries remain invariant under the action of a Lie group. In particular, the problem of writing down all local Riemannian invariants reduces to understanding the invariants of the orthogonal group.

In more recent times, a major program was initiated by C. Fefferman in [16] aimed at finding all scalar local invariants in CR geometry. This was motivated by the problem of understanding the local invariants which appear in the asymptotic expansions of the Bergman and Szegö kernels of strictly pseudo-convex CR manifolds, in a similar way to which Riemannian invariants appear in the asymptotic expansion of the heat kernel; the study of the local invariants in the singularities of these kernels led to important breakthroughs in [8] and more recently by Hirachi in [24]. This program was later extended to conformal geometry in [17]. Both these geometries belong to a broader class of structures, the parabolic geometries; these are structures which admit a principal bundle with structure group a parabolic subgroup $P$ of a semi-simple Lie group $G$, and a Cartan connection on that principle bundle (see the introduction in [12]). An important question in the study of these structures is the problem of constructing all their local invariants, which can be thought of as the natural, intrinsic scalars of these structures.

In the context of conformal geometry, the first (modern) landmark in understanding local conformal invariants was the work of Fefferman and Graham in 1985 [17], where they introduced their ambient metric. This allows one to construct local conformal invariants of any order in

\footnotetext{
${ }^{3}$ These are sometimes called Weyl anomalies.

${ }^{4}$ See also interesting work of Boulanger in [10] on Weyl anomalies which satisfy the Wess-Zumino consistency conditions.
} 
odd dimensions, and up to order $\frac{n}{2}$ in even dimensions. A natural question is then whether all invariants arise via this construction.

The subsequent work of Bailey-Eastwood-Graham [8] proved that this is indeed true in odd dimensions; in even dimensions, they proved that the result holds when the weight (in absolute value) is bounded by the dimension. The ambient metric construction in even dimensions was recently extended by Graham-Hirachi, [22]; this enables them to identify in a satisfactory manner all local conformal invariants, even when the weight (in absolute value) exceeds the dimension.

An alternative construction of local conformal invariants can be obtained via the tractor calculus introduced by Bailey-Eastwood-Gover in [7]. This construction bears a strong resemblance to the Cartan conformal connection, and to the work of T.Y. Thomas in 1934 [25]. The tractor calculus has proven to be very universal; tractor bundles have been constructed [12] for an entire class of parabolic geometries. The relation between the conformal tractor calculus and the Fefferman-Graham ambient metric has been elucidated in [13].

The present work, while pertaining to the question above (given that it ultimately deals with the algebraic form of local Riemannian and conformal invariants), nonetheless addresses a different type of problem. We here consider Riemannian invariants $P(g)$ for which the integral $\int_{M^{n}} P(g) d V_{g}$ remains invariant under conformal changes of the underlying metric. We then seek to understand the algebraic form of the integrand $P(g)$, ultimately proving that it can be de-composed in the way that Deser and Schwimmer asserted. It is thus not surprising that the prior work on the construction and understanding of local conformal invariants plays a central role in this endeavor, both in [3] and in the present paper.

Index theory. Questions similar to the Deser-Schwimmer conjecture arise naturally in index theory; a good reference for such questions is [9]. For example, in the heat kernel proof of the index theorem (for Dirac operators) by Atiyah-Bott-Patodi [6], the authors were led to consider integrals arising in the (integrated) expansion of the heat kernel over Riemannian manifolds of general Dirac operators, and sought to understand the local structure of the integrand ${ }^{5}$. In that setting, however, the fact that one deals with a specific integrand which arises in the heat kernel expansion plays a key role in the understanding of its local structure. This is true both of the original proof of Patodi, Atiyah-Bott-Patodi [6] and of their subsequent simplifications and generalizations by Getzler, Berline-Getzler-Vergne, see [9].

The closest analogous problem to the one considered here is the work of Gilkey and BransonGilkey-Pohjanpelto [19, 11]. In [19], Gilkey considered Riemannian invariants $P(g)$ for which the integral $\int_{M^{n}} P(g) d V_{g}$ on any given (topological) manifold $M^{n}$ has a given value, independent of the metric $g$. He proved that $P(g)$ must then be equal to a divergence, plus possibly a multiple of the Chern-Gauss-Bonnet integrand, if the weight of $P(g)$ agrees with the dimension in absolute value. In [11] the authors considered the problem of Deser-Schwimmer for locally conformaly flat metrics and derived the same decomposition (for locally conformaly flat metrics) as in [19]. Although these two results can be considered precursors of ours, the methods there are entirely different from the ones here; it is highly unclear whether the methods of $[19,11]$ could be applied to the problem at hand.

We next review the postponed claims that we will be proving here.

In Section 2 we recall from [3] that $P(g)$ is thought of as a linear combination of complete contractions involving factors of two types: iterated covariant derivatives of the Weyl tensor and iterated covariant derivatives of the Schouten tensor ${ }^{6}$. In other words, we write $P(g)=$

\footnotetext{
${ }^{5}$ We note that the geometric setting in [6] is more general than the one in the Deser-Schwimmer conjecture: In particular one considers vector bundles, equipped with an auxiliary connection, over compact Riemannian manifolds; the local invariants thus depend both on the curvature of the Riemannian metric and the curvature of the connection.

${ }^{6}$ We refer the reader to the introduction of [3] for a definition of these classical tensors. The Weyl tensor is the trace-free part of the curvature tensor and is conformally invariant, while the Schouten tensor is a trace-adjustment of the Ricci tensor (and is not conformally invariant).
} 
$\sum_{l \in L} a_{l} C^{l}(g)$, where each $C^{l}(g)$ is in the form (1.3) below. (Recall from the introduction in [3] that all complete contractions in $P(g)$ are assumed to have weight $-n$.)

In Section 2 we prove certain special cases of Propositions 2.3.1, 2.3.2 from Chapter 2 in [3] that were postponed to the present paper; these are contained in the next lemma:

Lemma 1.1. Let $P(g)$ be a Riemannian invariant of weight $-n$ such that the integral $\int_{M^{n}} P(g) d V_{g}$ is a global conformal invariant. Assume that $P(g)$ is in the form $P(g)=\sum_{l \in L} a_{l} C^{l}(g)$, where each $C^{l}(g)$ is a complete contraction:

$$
\operatorname{contr}\left(\nabla^{(m)} W \otimes \cdots \otimes \nabla^{\left(m^{\prime}\right)} W \otimes \nabla^{(a)} P \otimes \cdots \otimes \nabla^{\left(a^{\prime}\right)} P\right) .
$$

Let $\sigma>0$ be the minimum number of factors among the complete contractions $C^{l}(g), l \in L$. Denote by $L_{\sigma} \subset L$ the corresponding index set. We then claim that if $\sigma \leq 2$, there exists a local conformal invariant $W(g)$ and a Riemannian vector field $T^{i}(g)$ such that:

$$
P(g)=\operatorname{div}_{i} T^{i}(g)+W(g)+\sum_{l \in L^{\prime}} a_{l} C^{l}(g),
$$

where each $C^{l}(g), l \in L^{\prime}$ in the r.h.s. is in the form (1.3) and has at least $\sigma+1$ factors in total.

In Section 3 we prove the remaining claims for Lemmas 2.5.4, 2.5.5 from Chapter 2 in [3].

Recall the setting and claim of Lemma 2.5.4 in [3]. We recall some definitions. Recall first that given a $P(g)$ for which $\int_{M^{n}} P(g) d V_{g}$ is a global conformal invariant, we let $I_{g}^{s}(\phi)$ to be the $s^{t h}$ conformal variation of $P(g): I_{g}^{s}(\phi):=\left.\frac{d^{s}}{d t^{s}}\right|_{t=0} e^{n t \phi} P\left(e^{2 t \phi} g\right) . I_{g}^{s}\left(\psi_{1}, \ldots, \psi_{s}\right)$ is obtained from $I_{g}^{s}(\phi)$ via complete polarization:

$$
I_{g}^{s}\left(\psi_{1}, \ldots, \psi_{s}\right):=\left.\frac{d^{s}}{d \lambda_{1} \cdots d \lambda_{s}}\right|_{\lambda_{1}=0, \ldots, \lambda_{s}=0} I_{g}^{s}\left(\lambda_{1} \cdot \psi_{1}+\cdots+\lambda_{s} \cdot \psi_{s}\right) .
$$

We recall that since $\int_{M^{n}} P(g) d V_{g}$ is a global conformal invariant,

$$
\int_{M^{n}} I_{g}^{s}\left(\psi_{1}, \ldots, \psi_{s}\right) d V_{g}=0
$$

for all $\psi_{1}, \ldots, \psi_{s} \in \mathcal{C}^{\infty}\left(M^{n}\right)$ and all metrics $g$. We also recall that by virtue of the transformation laws of the curvature tensor and the Levi-Civita connections under conformal transformations, $I_{g}^{s}\left(\psi_{1}, \ldots, \psi_{s}\right)$ can be expressed as a linear combination of complete contractions in the form:

$$
\begin{aligned}
\operatorname{contr} & \left(\nabla^{f_{1} \ldots f_{y}} \nabla^{\left(m_{1}\right)} R_{i j k l} \otimes \cdots \otimes \nabla^{g_{1} \ldots g_{p}} \nabla^{\left(m_{r}\right)} R_{i j k l}\right. \\
& \otimes \nabla^{y_{1} \ldots y_{w}} \nabla^{\left(d_{1}\right)} \operatorname{Ric}_{i j} \otimes \cdots \otimes \nabla^{x_{1} \ldots x_{p}} \nabla^{\left(d_{q}\right)} \operatorname{Ric}_{i j} \\
& \left.\otimes \nabla^{a_{1} \ldots a_{t_{1}}} \nabla^{\left(u_{1}\right)} \psi_{1} \otimes \cdots \otimes \nabla^{c_{1} \ldots c_{t_{s}}} \nabla^{\left(u_{s}\right)} \psi_{s}\right),
\end{aligned}
$$

with the conventions introduced in Chapter 2 in $[3]^{7}$. In each factor $\nabla^{f_{1} \ldots f_{y}} \nabla_{r_{1} \ldots r_{m}}^{(m)} R_{i j k l}$, each of the upper indices ${ }^{f_{1}}, \ldots, f_{y}$ contracts against one of the indices $r_{1}, \ldots, l$, while no two of the indices $r_{1}, \ldots, l$ contract between themselves. On the other hand, for each factor $\nabla^{y_{1} \ldots y_{t}} \nabla_{a_{1} \ldots a_{u}}^{(u)} \psi_{h}$, each of the upper indices ${ }^{y_{1}}, \ldots,{ }^{y_{t}}$ contracts against one of the indices $a_{1}, \ldots, a_{u}$. Moreover, none of the indices $a_{1}, \ldots, a_{u}$ contract between themselves. For the factors $\nabla^{x_{1} \ldots x_{p}} \nabla_{t_{1} \ldots t_{u}}^{(u)} \mathrm{Ric}_{i j}$, we impose the condition that each of the upper indices ${ }^{x_{1}}, \ldots,{ }^{x_{p}}$ must contract against one of the lower indices $t_{1}, \ldots, t_{u}, i, j$. Moreover, we impose the restriction that none of the indices $t_{1}, \ldots, t_{u}, i, j$ contract between themselves (this assumption can be made by virtue of the contracted second Bianchi identity). We recall some important definitions:

\footnotetext{
${ }^{7}$ Note in particular that $r$ is the number of factors $\nabla^{(m)} R_{i j k l}, q$ is the number of factors $\nabla^{(d)}$ Ric, and $s$ is the number of factors $\nabla^{(u)} \psi_{h}$.
} 
Definition 1.1. We recall that for complete contractions in the form (1.4), $\delta$ stands for the number of internal contractions ${ }^{8}$, where we are also counting the internal contraction $\left({ }^{a},{ }_{a}\right)$ in each factor $\nabla^{(p)} \operatorname{Ric}_{i j}=\nabla^{(p)} R^{a}{ }_{i a j}$ and the two internal contractions in each factor $R=R_{a b}^{a b}$. We also recall that for such complete contractions, $|\Delta|$ stands for the total number of factors $\Delta \psi_{h}$ and $R$ in (1.4). We also recall that a complete contraction in the form (1.4) is called "acceptable" if each factor $\psi_{h}$ is differentiated at least twice. Also, partial contractions in he form (1.4) are called acceptable is each $\psi_{h}$ is differentiated at least twice ${ }^{9}$.

Furthermore, we recall that among the complete contractions $\operatorname{contr}(\cdots)$ in $I_{g}^{s}\left(\psi_{1}, \ldots, \psi_{s}\right)$ with the minimum number $\sigma$ of factors in total, $\mu$ is the minimum number of internal contractions.

We also recall a definition we have introduced for complete contractions of in the form (1.4):

Definition 1.2. Consider any complete contraction $C_{g}\left(\psi_{1}, \ldots, \psi_{a}\right)$ in the form (1.4) with $\sigma$ factors. If $C_{g}\left(\psi_{1}, \ldots, \psi_{s}\right)$ has $q=0$ and $\delta=\mu$ it will be called a target. If $C_{g}^{l}\left(\psi_{1}, \ldots, \psi_{s}\right)$ has $q=0$ and $\delta>\mu$, it will be called a contributor.

If $C_{g}\left(\psi_{1}, \ldots, \psi_{s}\right)$ has $q>0$ and $\delta>\mu$ we call it 1-cumbersome. We call $C_{g}\left(\psi_{1}, \ldots, \psi_{s}\right)$ 2-cumbersome if it has $q>0$ and $\delta=\mu$ and the feature that each factor $\nabla^{a_{1} \ldots a_{t}} \nabla_{r_{1} \ldots r_{p}}^{(p)} \operatorname{Ric}_{i j}$ has $t>0$ and the index ${ }_{j}$ is contracting against one of the indices ${ }^{a_{1}}, \ldots,{ }^{a_{t}}$.

Finally, when we say $C_{g}\left(\psi_{1}, \ldots, \psi_{s}\right)$ is "cumbersome", we will mean it is either 1-cumbersome or 2-cumbersome.

Definition 1.3. We denote by $\sum_{j \in J} a_{j} C_{g}^{j}\left(\psi_{1}, \ldots, \psi_{s}\right)$ a generic linear combination of contributors. We denote by $\sum_{f \in F} a_{f} C_{g}^{f}\left(\psi_{1}, \ldots, \psi_{s}\right)$ a generic linear combination of cumbersome complete contractions.

We have then derived in Chapter 2 in [3] that $I_{g}^{s}\left(\psi_{1}, \ldots, \psi_{s}\right)$ can be expressed in the form:

$$
\begin{aligned}
I_{g}^{s}\left(\psi_{1}, \ldots, \psi_{s}\right)= & \sum_{l \in L_{\mu}} a_{l} C_{g}^{l, \iota}\left(\psi_{1}, \ldots, \psi_{s}\right)+\sum_{j \in J} a_{j} C_{g}^{j}\left(\psi_{1}, \ldots, \psi_{s}\right) \\
& +\sum_{f \in F} a_{f} C_{g}^{f}\left(\psi_{1}, \ldots, \psi_{s}\right)+\text { (Junk). }
\end{aligned}
$$

(We recall from Chapter 2 in [3] that $\sum_{l \in L_{\mu}} a_{l} C_{g}^{l, l}\left(\psi_{1}, \ldots, \psi_{s}\right)$ in the above is a specific linear combination of targets, which is in precise correspondence with a specific linear combination of terms in $P(g)$; the precise form of this correspondence is not important for the present paper, so we do not dwell on this further.)

We also recall from Chapter 2 in [3] that for each $q, 1 \leq q \leq \sigma-s, F^{q} \subset F$ stands for the index set of complete contractions with precisely $q$ factors $\nabla^{(p)}$ Ric or $R$. Recall that for each complete contraction in the form (1.4) we have denoted by $|\Delta|$ the number of factors in one of the forms $\Delta \psi_{h}, R$. For each index set $F^{q}$ above, let us denote by $F^{q, *} \subset F^{q}$ the index set of complete contractions with $|\Delta| \geq \sigma-2$, and $F^{*}=\bigcup_{q>0} F^{q, *}$.

Important remark. We recall a remark made in Chapter 2 in [3] where we noted that any $C_{g}^{f}\left(\psi_{1}, \ldots, \psi_{s}\right)$ with $\alpha>0$ factors $R$ (of the scalar curvature) will have $\delta \geq \mu+2 \alpha$.

The missing claim needed to derive Lemma 2.5.4 in [3] is then the following:

Lemma 1.2. There exists a linear combination of vector fields (indexed in $H$ below), each in the form (1.4) with $\sigma$ factors ${ }^{10}$, so that modulo complete contractions of length $>\sigma$ :

$$
\sum_{f \in F^{*}} a_{f} C_{g}^{f}\left(\psi_{1}, \ldots, \psi_{s}\right)-\operatorname{div}_{i} \sum_{h \in H} a_{h} C_{g}^{h, i}\left(\psi_{1}, \ldots, \psi_{s}\right)=\sum_{y \in Y} a_{y} C_{g}^{y}\left(\psi_{1}, \ldots, \psi_{s}\right),
$$

\footnotetext{
${ }^{8}$ We recall that an "internal contraction" is a pair of indices that belong to the same factor and contract against each other.

${ }^{9}$ For future reference, we also recall that a partial contraction with one free index is also called a vector field.

${ }^{10}$ The vector fields in question are partial contractions in the form (1.4) with one free index and with weight $-n+1$.
} 
where the complete contractions indexed in $Y$ are in the form (1.4) with length $\sigma$, and satisfy all the properties of the sublinear combination $\sum_{f \in F} \cdots$ but in addition have $|\Delta| \leq \sigma-3$.

The setting and remaining claims for Lemma 2.5.5 in [3]. Recall that in the setting of Lemma 2.5.5, our point of reference is a linear combination:

$$
Y_{g}\left(\psi_{1}, \ldots, \psi_{s}\right)=\sum_{l \in L_{\mu}} a_{l} C_{g}^{l, \iota}\left(\psi_{1}, \ldots, \psi_{s}\right)+\sum_{j \in J} a_{j} C_{g}^{j}\left(\psi_{1}, \ldots, \psi_{s}\right)+(\mathrm{Junk}),
$$

with the same conventions as in the ones under (1.5). The remaining claims of Lemma 2.5.5 (the proof of which was deferred to this paper) are as follows:

Denote by $L_{\mu}^{*} \subset L_{\mu}, J^{*} \subset J$ the index sets of complete contractions in $Y_{g}$ with $|\Delta| \geq \sigma-2$, among the complete contractions indexed in $L_{\mu}, J$ respectively.

Lemma 1.3. We claim that there exists a linear combination of vector fields (indexed in $H$ below), with length $\sigma$, in the form (1.4) without factors $\nabla^{(p)} \operatorname{Ric}, R$ and with $\delta=\mu$ so that:

$$
\begin{aligned}
& {\left[\sum_{l \in L_{\mu}^{*}} a_{l} C_{g}^{l, i_{1} \ldots i_{\mu}}\left(\psi_{1}, \ldots, \psi_{s}\right)-\operatorname{div}_{i} \sum_{h \in H} a_{h} C_{g}^{h, i \mid i_{1} \ldots i_{\mu}}\left(\psi_{1}, \ldots, \psi_{s}\right)\right] \nabla_{i_{1}} v \cdots \nabla_{i_{\mu}} v} \\
& \quad=\sum_{l \in \bar{L}} a_{l} C_{g}^{l, i_{1} \ldots i_{\mu}}\left(\psi_{1}, \ldots, \psi_{s}\right) \nabla_{i_{1}} v \cdots \nabla_{i_{\mu}} v
\end{aligned}
$$

where the complete contractions indexed in $\bar{L}$ are in the form (1.4) with no factors $\nabla^{(p)}$ Ric or $R$ and with $|\Delta| \leq \sigma-3$.

In the setting $L_{\mu}^{*}=\varnothing$, what remains to be shown to complete the proof of Lemma 2.5.5 in [3] is the following:

Lemma 1.4. Assume that $L_{\mu}^{*}=\varnothing$, and $J^{*}$ is as above. We then claim that there exists a linear combination of vector fields (indexed in $H$ below) so that:

$$
\sum_{j \in J^{*}} a_{j} C_{g}^{j}\left(\psi_{1}, \ldots, \psi_{s}\right)-\operatorname{div}_{i} \sum_{h \in H} a_{h} C_{g}^{h, i}\left(\psi_{1}, \ldots, \psi_{s}\right)=\sum_{y \in Y^{\prime}} a_{y} C_{g}^{y}\left(\psi_{1}, \ldots, \psi_{s}\right),
$$

where the complete contractions indexed in $Y^{\prime}$ are in the form (1.4) with length $\sigma$, with no factors $\nabla^{(p)} \operatorname{Ric}$ or $R$ and have $\delta \geq \mu+1$ and in addition satisfy $|\Delta| \leq \sigma-3$.

The setting and claims of Lemma 2.5.3 in [3]. Recall that $P(g)=\sum_{l \in L} a_{l} C^{l}(g)$ is assumed to be a linear combination of complete contractions in the form (1.3). Recall that $\sigma$ is the minimum number of factors (in total) among all complete contractions $C^{l}(g) ; L_{\sigma} \subset L$ is the corresponding index set. Also, $s>0$ is the maximum number of factors $\nabla^{(a)} P$ among the complete contractions $C^{l}(g)$ indexed in $L_{\sigma}$; we denote the corresponding index set by $\Theta_{s} \subset L^{\prime}$. We have defined $\left.P(g)\right|_{\Theta_{s}}:=\sum_{l \in \Theta_{s}} a_{l} C^{l}(g)$.

Special definition. If $s=\sigma-2$ then $\left.P(g)\right|_{\Theta_{s}}$ is "good" if the only complete contraction in $\left.P(g)\right|_{\Theta_{s}}$ with $\sigma-2$ factors $P_{a}^{a}$ is of the form (const) $\cdot \operatorname{contr}\left(\Delta^{\frac{n}{2}-\sigma-2} \nabla^{i l} W_{i j k l} \otimes \nabla^{i^{\prime} l^{\prime}} W_{i^{\prime}}{ }^{j k}{ }_{l^{\prime}} \otimes\right.$ $\left(P_{a}^{a}\right)^{\sigma-2}$ ) (when $\sigma<\frac{n}{2}-1$ ) or (const) $\cdot \operatorname{contr}\left(\nabla^{l} W_{i j k l} \otimes \nabla_{l^{\prime}} W^{i j k l^{\prime}} \otimes\left(P_{a}^{a}\right)^{\sigma-2}\right)$ when $\sigma=\frac{n}{2}-1$. If $s=\sigma-1$, then $\left.P(g)\right|_{\Theta_{s}}$ is "good" if all complete contractions in $\left.P(g)\right|_{\Theta_{s}}$ have $\delta_{W}+\delta_{P}=\frac{n}{2}-1.11$

Lemma 2.5.3 in [3] claims:

\footnotetext{
${ }^{11}$ In other words, if there are complete contractions in $\left.P(g)\right|_{\Theta_{s}}$ with $\delta_{W}+\delta_{P}<\frac{n}{2}-1$ then $\left.P(g)\right|_{\Theta_{s}}$ is "good" if no complete contractions in $\left.P(g)\right|_{\Theta_{s}}$ have $\sigma-2$ factors $P_{a}^{a}$.
} 
Lemma 1.5. There exists a divergence $\operatorname{div}_{i} T^{i}(g)$ so that

$$
\left.P(g)\right|_{\Theta_{s}}-\operatorname{div}_{i} T^{i}(g)=\sum_{l \in \Theta_{s}^{\prime}} a_{l} C^{l}(g)+\sum_{t \in T} a_{t} C^{t}(g)
$$

Here each $C^{t}(g)$ is in the form (1.3) and has fewer than $s$ factors $\nabla^{(p)} P$. The complete contractions indexed in $\Theta_{s}^{\prime}$ are in the form (1.3) with s factors $\nabla^{(p)} P$ and moreover this linear combination is "good".

\section{Proof of Lemma 1.1}

We first observe that the claim is trivial when $\sigma=1$. In that case (modulo applying a curvature identity and introducing longer correction terms), the sublinear combination $\left.P(g)\right|_{1}$ will be $\left.P(g)\right|_{1}=$ (const) $\cdot \Delta^{\frac{n}{2}-1} R$ ( $R$ is the scalar curvature). Thus, $\left.P(g)\right|_{1}$ can be written as (const) $\nabla^{i}\left(\nabla_{i} \Delta^{\frac{n}{2}-2} R\right)$.

The case $\sigma=2$ is dealt with by explicitly constructing divergences of vector fields and long calculations of one local conformal invariant, using the Feffeman-Graham ambient metric, $[17,18]^{12}$. We first consider the terms in $\left.P(g)\right|_{2}$ with two factors $\nabla^{(m)} P_{a b}$. We will show that we can explicitly construct a divergence $\operatorname{div}_{i} T^{i}(g)$ so that:

$$
\left.P(g)\right|_{2}-\operatorname{div}_{i} T^{i}(g)=(\text { const })\left|\nabla^{\left(\frac{n}{2}-2\right)} P\right|^{2}+\sum_{h \in H} a_{h} C^{h}(g)+\sum_{w \in W} a_{w} C^{w}(g),
$$

where the terms indexed in $H$ have one factor $\nabla^{(m)} W_{i j k l}$ and one factor $\nabla^{\left(m^{\prime}\right)} P_{a b}$. The terms indexed in $W$ have two factors $\nabla^{(m)} W_{i j k l}$. (The above holds modulo longer complete contractions, as usual).

We explain how (2.1) is proven in detail, since the main idea will be used repeatedly throughout this section. Let us first recall a few classical identities, which can be found in [3].

Useful formulae. Firstly, antisymmetrizing the indices ${ }_{c},{ }_{a}$ in a factor $\nabla_{r_{1} \ldots r_{m} c}^{(m+1)} P_{a b},{ }^{13}$ gives rise to a Weyl tensor:

$$
\nabla_{r_{1} \ldots r_{m} c}^{(m+1)} P_{a b}-\nabla_{r_{1} \ldots r_{m} a}^{(m+1)} P_{c b}=\frac{1}{3-n} \nabla_{r_{1} \ldots r_{m} s}^{(m)} W_{c a b} s
$$

We also recall that the indices $i, j$ and $k, l$ in each tensor $\nabla_{r_{1} \ldots r_{M}}^{(m)} W_{i j k l}$ are anti-symmetric. Finally, we recall the "fake" second Bianchi identities from Chapter 2 in [3]. These are substitutes for the second Bianchi identity for the tensor $\nabla_{a} W_{i j k l}$.

Now, consider any complete contraction $C(g)$ involving exactly two factors, $\nabla^{(m)} P_{a b}$ and $\nabla^{\left(m^{\prime}\right)} P_{a^{\prime} b^{\prime}}$. So $C(g)=\operatorname{contr}\left(\nabla^{(m)} P_{a b} \otimes \nabla^{\left(m^{\prime}\right)} P_{a^{\prime} b^{\prime}}\right)$. We firstly show that by subtracting an explicitly constructed divergence ${ }^{14} \operatorname{div}_{i} T^{i}(g)=\sum_{h=1}^{K} \operatorname{div}_{i} C^{K, i}(g)$ we can write:

$$
C(g)-\operatorname{div}_{i} T^{i}(g)=C^{\prime}(g)
$$

(modulo terms with more than two factors), where $C^{\prime}(g)$ is some complete contraction involving two factors $T_{1}=\nabla^{\left(\frac{n}{2}-2\right)} P_{a b}, T_{2}=\nabla^{\left(\frac{n}{2}-2\right)} P_{a^{\prime} b^{\prime}}$ with the additional property that each of the $\frac{n}{2}$ indices in the first factor contract against an index in the second factor, and vice versa.

\footnotetext{
${ }^{12}$ We refer the reader to Chapter 2 in [3] for a review of the ambient metric and and of the algorithm we employ for computations.

${ }^{13}$ This is the $(m+1)$ st iterated covariant derivative of the Schouten tensor, see [3] for details.

${ }^{14}$ As allowed in the Deser-Schwimmer conjecture.
} 
Proof of (2.1). We construct the divergences needed for (2.3). Let us suppose that $C(g)$ has $M$ pairs of indices $(s, t)$ which belong to the same factor and contract against each other; we call such contractions "internal contractions". If $M=0 C(g)$ is in the desired form; thus we may assume that $M>0$. Firstly, by using the second contracted Bianchi identity $\nabla_{c} P_{a}^{a}=\nabla_{a} P_{c}^{a}$, we may assume that if one of the factors $\nabla^{(m)} P_{a b}$ in $C(g)$ has $m>0$, then the indices $a, b$ are not contracting against each other. After this, we construct the divergence by an iterative procedure. Pick out any pair of indices $s, t$ in $C(g)$ which contract against each other and belong to the same factor; assume without loss of generality that $s$ is a derivative index ${ }^{15}$. We then construct a partial contraction $C^{1, i}(g)$ out of $C(g)$ by erasing the (derivative) index ${ }_{s}$ and making the index $t$ into a free index ${ }^{i}$. Observe then that (modulo complete contractions with at least three factors), $C(g)-\operatorname{div}_{i} C^{1, i}(g)=\bar{C}(g)$, where $\bar{C}(g)$ has $M-1$ "internal contractions". Iterating this step $M-1$ more times, we derive (2.3).

Now, consider $C^{\prime}(g)$ and consider the indices ${ }_{a},{ }_{b}$ in the factor $T_{1}=\nabla^{\left(\frac{n}{2}-2\right)} P_{a b}$. They contract against two indices ( say $_{c}, d$ ) in the factor $T_{2}$. We then apply the curvature identity repeatedly and also the identity (2.2) to arrange that the indices $a, b$ in $T_{1}$ contract against the indices $c, d$ in $T_{2}$. The correction terms that arise by the application of the curvature identity have three factors. The correction terms arising from (2.2) will be in the generic form $\sum_{h \in H} a_{h} C^{h}(g)$ as described below (2.1).

Now, we will show that (const) $=0$ in $(2.1)$. We derive this easily. Consider $I_{g}^{2}(\phi)(:=$ $\left.\left.\frac{d^{2}}{d t^{2}}\right|_{t=0}\left[e^{n t \phi} P\left(e^{2 t \phi} g\right)\right]\right)$ and apply the super divergence formula to the above ${ }^{16}$. We derive that (const) $\cdot\left|\nabla^{\left(\frac{n}{2}\right)} \phi\right|^{2}=0$ (modulo longer terms). Therefore, (const) $=0$.

Thus, we may assume without loss of generality that

$$
\left.P(g)\right|_{2}=\sum_{h \in H} a_{h} C^{h}(g)+\sum_{w \in W} a_{w} C^{w}(g)
$$

with the same conventions introduced under (2.1).

We next claim that we can explicitly construct a divergence $\operatorname{div}_{i} T_{g}^{i}$ such that:

$$
\sum_{h \in H} a_{h} C^{h}(g)-\operatorname{div}_{i} T^{\prime i}(g)=\sum_{w \in W} a_{w} C^{w}(g),
$$

where the terms indexed in $W$ are of the generic form described above. The divergence needed for the above is constructed by the same technique as for (2.3): In each $C^{h}(g)$ we iteratively pick out the internal contractions in the factor $\nabla^{(m)} W_{i j k l}$, erase one (derivative) index in that contraction, (thus obtaining a partial contraction with one free index) and subtract the divergence of that partial contraction ${ }^{17}$. After repeating this process enough times so that no internal contraction is left in the factor $\nabla^{(m)} W_{i j k l}$, we end up with a formula:

$$
\sum_{h \in H} a_{h} C^{h}(g)-\operatorname{div}_{i} T^{\prime i}(g)=\sum_{r \in R} a_{r} C^{r}(g) .
$$

Here the complete contractions $C^{r}(g)$ in the r.h.s. are in the form $\operatorname{contr}\left(\nabla^{(m)} W_{i j k l} \otimes \nabla^{(t)} P_{a b}\right)$, where all $m+4$ indices in the factor $\nabla^{(m)} W_{i j k l}$ contract against the other factor $\nabla^{(t)} P_{a b}$. Now,

\footnotetext{
${ }^{15}$ This assumption can be made by virtue of the previous sentence and since we are dealing with complete contractions of weight $-n$.

${ }^{16}$ See the algorithm at the end of [1].

${ }^{17}$ Sometimes, by abuse of language, we will refer to this subtraction of an explicit divergence as an "integration by parts".
} 
since the indices ${ }_{a},{ }_{b}$ in Schouten tensor are symmetric and the indices ${ }_{i},{ }_{j}$ and ${ }_{k}, l$ are antisymmetric, it follows from (2.2) and from the curvature identity that:

$$
\sum_{r \in R} a_{r} C^{r}(g)=\sum_{w \in W} a_{w} C^{w}(g),
$$

modulo complete contractions with three factors, which arise due to the curvature identity.

So we may assume without loss of generality that $\left.P(g)\right|_{2}$ consists of terms with two factors $\nabla^{(m)} W_{i j k l}$.

We can then again explicitly construct a divergence $\operatorname{div}_{i} T^{\prime \prime i}(g)$, in order to write (modulo longer complete contractions):

$$
\left.P(g)\right|_{2}-\operatorname{div}_{i} T^{\prime \prime i}(g)=\sum_{w \in W^{\prime}} a_{w} C_{g}^{w},
$$

where the terms in the r.h.s. have the additional feature that none of the two factors $\nabla^{(m)} W_{a b c d}$ have internal contractions. The divergence $\operatorname{div}_{i} T^{\prime \prime i}(g)$ is constructed by the iterative procedure used to prove (2.3). So we may assume without loss of generality that all terms in $\left.P(g)\right|_{2}$ have this property.

Finally, by just keeping track of the correction terms of the form $\nabla^{s} W_{s d f h} \otimes g$ in the "fake" second Bianchi identities ${ }^{18}$, we will prove that we can can explicitly construct a divergence $\operatorname{div}_{i} T^{i}(g)$ such that:

$$
\left.P(g)\right|_{2}=(\text { const })^{\prime} C^{*}(g)+\operatorname{div}_{i} T^{i}(g),
$$

where $C^{*}(g)$ is the complete contraction $\left|\nabla^{\left(\frac{n}{2}-2\right)} W_{a b c d}\right|^{2}$.

Proof of (2.4). By virtue of the anti-symmetry of the indices ${ }_{i}, j$ and $k, l$ and of the first Bianchi identity in the Weyl tensor $W_{i j k l}$, we see that (modulo introducing correction terms with three factors), $\left.P(g)\right|_{2}$ can be expressed in the form:

$$
\left.P(g)\right|_{2}=a \cdot\left|\nabla^{\left(\frac{n}{2}-2\right)} W_{a b c d}\right|^{2}+b \cdot C^{2}(g)+c \cdot C^{3}(g),
$$

where $C^{2}(g)$ is the complete contraction:

$$
\operatorname{contr}\left(\nabla_{r_{1} \ldots r_{\frac{n}{2}-3} s} W_{t j k l} \otimes \nabla^{r_{1} \ldots r_{\frac{n}{2}-3} t} W^{s j k l}\right)
$$

while $C^{3}(g)$ is the complete contraction:

$$
\operatorname{contr}\left(\nabla_{r_{1} \ldots r_{\frac{n}{2}-4} s u} W_{t j k y} \otimes \nabla^{r_{1} \ldots r_{\frac{n}{2}-4} t y} W^{s j k u}\right) .
$$

We then only have to apply the "fake" second Bianchi identities from Chapter 2 in [3] to derive that we can write:

$$
C^{2}(g)=\frac{1}{2}\left|\nabla^{\left(\frac{n}{2}-2\right)} W_{a b c d}\right|^{2}+\frac{1}{n-3}\left|\nabla^{\left(\frac{n}{2}-3\right)} \nabla^{s} W_{s j k l}\right|^{2} .
$$

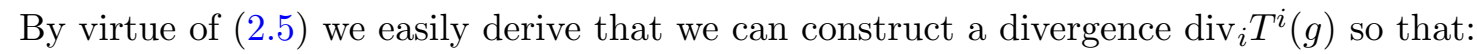

$$
C^{2}(g)-\operatorname{div}_{i} T^{i}(g)=\frac{n-3}{2(n-4)}\left|\nabla^{\left(\frac{n}{2}-2\right)} W_{a b c d}\right|^{2}
$$

(notice the constant is strictly positive) ${ }^{19}$.

\footnotetext{
${ }^{18}$ These are presented in Chapter 2 in [3].

${ }^{19}$ The divergence is constructed in the same way as in the proof of (2.3). We just "integrate by parts" the two internal contractions ${ }^{s},{ }_{s}$ in the two factors in $\left|\nabla^{\left(\frac{n}{2}-3\right)} \nabla^{s} W_{s j k l}\right|^{2}$.
} 
In order to deal with the complete contraction $C^{3}(g)$, we will note another useful identity. Let $\bar{C}^{3}(g)$ stand for the complete contraction $\left(\nabla_{r_{1} \ldots r_{\frac{n}{2}-4} a} W_{i j k l} \otimes \nabla^{r_{1} \ldots r_{\frac{n}{2}-4} i s} W^{a j k}{ }_{s}\right)$; we also denote by $C^{\prime 3}(g)$ the complete contraction: $\left(\nabla_{r_{1} \ldots r_{\frac{n}{2}-3} s} W_{i j k}{ }^{s} \otimes \nabla^{r_{1} \ldots r_{\frac{n}{2}-3} t} W^{i j k} t\right)$. We then calculate:

$$
\bar{C}^{3}(g)=\frac{1}{2} C^{\prime 3}(g)
$$

Thus, using the above we derive as before that we can explicitly construct a divergence $\operatorname{div}_{i} T^{\prime i}(g)$ such that:

$$
C^{3}(g)-\operatorname{div}_{i} T^{\prime i}(g)=\frac{1}{2} C^{2}(g) .
$$

Therefore, we derive that modulo subtracting a divergence $\operatorname{div}_{i} T^{i}(g)$ from $\left.P(g)\right|_{2}$, we may assume that $\left.P(g)\right|_{2}$ is in the form $\left.P(g)\right|_{2}=($ const $) \cdot\left|\nabla^{\left(\frac{n}{2}-2\right)} W\right|^{2}$.

A construction in the Fefferman-Graham ambient metric. The reader is referred to the discussion on the Fefferman-Graham ambient metric from Subsection 3.2.1 in [3]. We consider the local conformal invariant: $\tilde{C}(g)=\Delta_{\tilde{g}}^{\frac{n}{2}-2}|\tilde{R}|_{\tilde{g}}^{2}$. Here we think of $|\tilde{R}|_{\tilde{g}}^{2}$ as the product $\tilde{g}^{a \alpha} \tilde{g}^{b \beta} \tilde{g}^{c \gamma} \tilde{g}^{d \delta} \tilde{R}_{a b c d} \tilde{R}_{\alpha \beta \gamma \delta}$, where $\tilde{R}$ is the ambient curvature tensor, $\tilde{g}$ is the ambient metric tensor and $\Delta_{\tilde{g}}$ is the Laplace-Beltrami operator associated to $\tilde{g}$.

We will show that there exists an explicit divergence $\operatorname{div}_{i} T^{i}(g)$, so that modulo terms of length $\geq 3$ :

$$
\tilde{C}(g)=(\text { const }) C^{*}(g)+\operatorname{div}_{i} T^{i}(g) .
$$

(Here the constant (const) is non-zero.) If we can prove the above then in view of (2.4) our claim will clearly follow.

Proof of (2.8). We recall certain facts about the ambient metric The reader is referred to [18], or Chapter 2 in [3]. Firstly recall that given any point $P \in M$ and coordinates $\left\{x^{1}, \ldots, x^{n}\right\}$, there is a canonical coordinate system $\left\{t=x^{0}, x^{1}, \ldots, x^{n}, \rho\right\}$ around the image $\tilde{P}$ of $P$ in the ambient manifold $(\tilde{G}, \tilde{g})$. Recall that the local conformal invariant is evaluated at the point $\tilde{P}$, and $t(\tilde{P})=1, \rho(\tilde{P})=0$.

Recall also that the vector fields $X^{0}, X^{1}, \ldots, X^{n}, X^{\infty}$ are the coordinate vector fields $\frac{\partial}{\partial x^{0}}, \ldots$, $\frac{\partial}{\partial x^{n}}, \frac{\partial}{\partial \rho}$. Further, when we give values $0,1, \ldots, n, \infty$ to indices of tensors that appear further down, these values correspond to the coordinate frame above.

Now, we recall some basic facts regarding the Taylor expansion of the ambient metric $\tilde{g}$ at $\tilde{P}$. Firstly, that $\partial_{\infty} \tilde{g}^{a b}=-2 P^{a b}$ if $1 \leq a, b \leq n$ and $\partial_{\infty} \tilde{g}^{\infty \infty}=-2$. These are the only non-zero components of the matrix $\partial_{\infty} \tilde{g}^{c d}$ (with raised indices).

Now, in order to state our next claim, we recall that $\tilde{\Gamma}_{a b}^{c}$ stand for the Christoffel symbols of the ambient metric. We recall that if $0 \leq a, b, c \leq n$ then $\partial_{\infty} \tilde{\Gamma}_{a b}^{c}=F_{a b}^{c}(R)$, where the expression in the r.h.s. stands for a tensor (in the indices $a,{ }_{b},{ }^{c}$ ) involving at least one (possibly internally contracted) factor of the curvature tensor. On the other hand, we also recall that if $1 \leq a, b, c \leq n$ then $\tilde{\Gamma}_{a b}^{c}=\Gamma_{a b}^{c}$ (the r.h.s. stands for the Christoffel symbol of the metric $g$ ). Finally, if $1 \leq a, b \leq n$ then $\tilde{\Gamma}_{a b}^{\infty}=-g_{a b}$. Furthermore, we will use the fact that $\partial_{\infty} \tilde{g}_{a b}=2 P_{a b}$ and also the formula (3.21) from [18]:

$$
\partial_{\infty}^{s} \tilde{g}_{i j}=\frac{2}{(4-n) \cdots(2 s-n)}\left[\Delta^{s-1} P_{i j}-\Delta^{s-2} \nabla_{i j} P_{a}^{a}\right]+Q(R)
$$


(for $s \geq 2$; all other components $\partial_{\infty \ldots \infty}^{(s)} \tilde{g}_{A b}$ vanish if $A=0, \infty$ and $\left.s \geq 2 ; b=0,1, \ldots, n, \infty\right)^{20}$. We calculate that for $1 \leq i, j, k, l \leq n$ and $1 \leq \alpha \leq \frac{n}{2}-2$ :

$$
\partial_{\infty}^{\alpha} \tilde{R}_{i j k l}=(-1)^{\alpha-1} \frac{1}{(n-3)(n-4) \cdots(n-2 \alpha)} \Delta^{\alpha-1}\left[\nabla_{j}^{t} W_{t i k l}-\nabla_{i}^{t} W_{t j k l}\right]+Q(R)
$$

(where if $\alpha=1$ then the constant above is $\frac{1}{n-3}$ ). Furthermore for $0 \leq \alpha \leq \frac{n}{2}-3$ :

$$
\partial_{\infty}^{\alpha} \tilde{R}_{\infty j k l}=(-1)^{\alpha-1} \frac{1}{(n-3)(n-4) \cdots(n-2-2 \alpha)} \Delta^{\alpha} \nabla^{s} W_{s j k l}+Q(R)
$$

(where if $\alpha=0$ then the constant above is $\frac{1}{n-3}$ ). Moreover for $0 \leq \alpha \leq \frac{n}{2}-4$ :

$$
\partial_{\infty}^{\alpha} \tilde{R}_{\infty j k \infty}=(-1)^{\alpha} \frac{1}{(n-3)(n-4) \cdots(n-4-2 \alpha)} \Delta^{\alpha} \nabla^{i l} W_{i j k l}+Q(R) .
$$

(The left-hand sides of the above are also known as Graham's extended obstruction tensors, see [20] for a detailed study of these tensors.)

Next, two calculations. Let $F(\tilde{g})$ be any Riemannian invariant in the ambient metric $\tilde{g}$, with weight $w$. (In particular, $F(\tilde{g})$ will have homogeneity $-w$ in $t$.) Then we calculate:

$$
\partial_{\infty}^{k} \Delta_{\tilde{g}}[F(\tilde{g})]=\Delta_{g} L(g)+(2 w+n-2 k) \partial_{\infty}^{k+1} F(\tilde{g})+Q(R) .
$$

$L(g)$ is a Riemannian invariant of the metric $g$, not the ambient metric $\tilde{g}$, with weight $w-2 k$. $Q(R)$ is a linear combination of complete contractions in the iterated covariant derivatives of the curvature tensor, and each complete contraction involves at least two such curvature terms.

A word regarding the derivation of the above: By the form of the ambient metric (see in particular page 20 in [18]), we derive that at any point on the ambient manifold,

$$
\begin{aligned}
\Delta_{\tilde{g}} F(\tilde{g})= & \sum_{i, j=1}^{n} \tilde{g}^{i j} \nabla_{i j}^{(2)} F(\tilde{g})+2 t^{-1} \tilde{\nabla}_{0 \infty}^{(2)} F(\tilde{g})-2 t^{2} \rho \tilde{\nabla}_{\infty \infty}^{(2)} F(\tilde{g}) \\
= & \sum_{i, j=1}^{n} \tilde{g}^{i j}\left(\partial_{i j}^{(2)}-\sum_{k=1}^{n} \tilde{\Gamma}_{i j}^{k} \partial_{k}\right) F(\tilde{g})-\left[\sum_{i, j=1}^{n} \tilde{g}^{i j} \tilde{\Gamma}_{i j}^{0} \partial_{0}+\sum_{i, j=1}^{n} \tilde{g}^{i j} \tilde{\Gamma}_{i j}^{\infty} \partial_{\infty}\right] F(\tilde{g}) \\
& +2 w t^{-1} \partial_{\infty} F(\tilde{g})-2 t^{2} \rho \partial_{\infty \infty}^{(2)} F(\tilde{g}) .
\end{aligned}
$$

Thus, if we take the $k^{\text {th }}$ derivative $\partial_{\infty \ldots \infty}^{(k)}$ of the above equation and then evaluate at $t=1$, $\rho=0$, we obtain the r.h.s. of (2.12) as follows: the term $\Delta_{g} L(g)$ arises when all $k$ derivatives $\partial_{\infty}$ hit the factor $F(\tilde{g})$ in $\sum_{i, j=1}^{n} \tilde{g}^{i j} \partial_{i j}^{(2)} F(\tilde{g})$. The coefficient $2 w$ arises from the term $\partial_{0 \infty}^{(2)} F(\tilde{g})$ in $\tilde{\nabla}_{0 \infty}^{(2)} F(\tilde{g})$, due to the homogeneity $w$ of $F(\tilde{g})$ in $x^{0}=t$. The coefficient $+n$ arises from the term $-\sum_{i, j=1}^{n} \tilde{g}^{i j} \tilde{\Gamma}_{i j}^{\infty} \partial_{\infty}$. Finally, the term $-2 k$ arises when exactly one of the $k$ derivatives $\partial_{\infty}$ hits the coefficient $-2 t^{2} \rho$ of the expression $\partial_{\infty \infty}^{(2)} F(\tilde{g})$. All other terms that arise are in the form $Q(R)$.

Now, we can iteratively apply the above formula to obtain a useful expression for $\Delta_{\tilde{g}}^{\frac{n}{2}-2}\left|\tilde{R}_{i j k l}\right|_{\tilde{g}}^{2}$; we consider $\Delta_{\tilde{g}}\left(\Delta_{\tilde{g}}\left(\ldots\left(\left.\left|\tilde{R}_{i j k l}\right|\right|_{\tilde{g}} ^{2}\right) \ldots\right)\right.$, and we replace each of the $\Delta_{\tilde{g}}$ 's according to $(2.12)$, from left to right. The resulting equation is:

$$
\tilde{C}(g)=(\text { const })^{\prime} \cdot \partial_{\infty}^{\frac{n}{2}-2}\left(\left|\tilde{R}_{i j k l}\right|_{\tilde{g}}^{2}\right)+\operatorname{Cubic}(R)+\Delta_{g} L(g) .
$$

\footnotetext{
${ }^{20} Q(R)$ stands for a linear combination of partial contractions involving at least two curvature terms, as usual.
} 
Here the constant (const) ${ }^{\prime}$ is non-zero; this is because at each application of the identity (2.12) we have $-w+2 k+2=n$, and $w$ takes on the values $-n+2, \ldots,-4$; thus each factor in the product is non-zero. $\operatorname{Cubic}(R)$ is a linear combination of Riemannian invariants with at least three factors. $L(g)$ is a Riemannian invariant (of the base metric $g$ ) of weight $-n+2$.

Now, using formulas $(2.9),(2.10),(2.11)$ and also the formula $\partial_{\infty} \tilde{g}^{\infty \infty}=-2$, we derive that:

$$
\begin{aligned}
& \partial_{\infty}^{\frac{n}{2}-2}\left(\left|\tilde{R}_{i j k l}\right| \begin{array}{l}
2 \\
\tilde{g}
\end{array}\right)=(-1)^{\frac{n}{2}}\left\{2 \cdot 2 \frac{1}{(n-3)(n-4) \cdots 4} W_{i j k l} \Delta^{\frac{n}{2}-3} \nabla_{t}^{i} W^{t j k l}\right. \\
&+2 \sum_{x=1}^{\frac{n}{2}-3}\left(\begin{array}{c}
\frac{n}{2}-2 \\
x
\end{array}\right)\left[\frac{1}{(n-3)(n-4) \cdots(n-2 x)}\right] \\
& \times\left[\frac{1}{(n-3)(n-4) \cdots(4+2 x)}\right] \Delta^{x} \nabla^{a s} W_{s j k l} \Delta^{\frac{n}{2}-2-x} \nabla_{a t} W^{t j k l}+\sum_{f \in F} a_{f} C^{f}(g) \\
&+\sum_{x=0} 2 \cdot 4\left(\begin{array}{c}
\frac{n}{2}-2 \\
1
\end{array}\right) \cdot\left(\begin{array}{c}
\frac{n}{2}-3 \\
x
\end{array}\right) \cdot\left[\frac{1}{(n-3)(n-4) \cdots(n-2 x-2)}\right] \\
& \times\left[\frac{1}{(n-3)(n-4) \ldots(4+2 x)}\right] \Delta^{x} \nabla^{s} W_{s j k l} \Delta^{\frac{n}{2}-3-x} \nabla_{t} W^{t j k l} \\
&+\sum_{x=0}^{\frac{n}{2}-4} 2\left(\begin{array}{c}
\frac{n}{2}-2 \\
1
\end{array}\right) 2 \cdot 2 \cdot\left(\begin{array}{c}
\frac{n}{2}-3 \\
1
\end{array}\right) \cdot\left(\begin{array}{l}
\frac{n}{2}-4 \\
x
\end{array}\right] \cdot\left[\frac{1}{(n-3)(n-4) \cdot(4+2 x)}\right] \\
& \times\left[\frac{1}{(n-3)(n-4) \ldots(n-4-2 x)}\right] \\
& \times \Delta^{x} \nabla^{s t} W_{s j t l} \Delta^{\frac{n}{2}-4-x} \nabla_{s^{\prime} t^{\prime}} W^{s^{\prime} j t^{\prime} l} \\
&
\end{aligned}
$$

Here $\operatorname{Cubic}(R)$ stands for a generic linear combination of partial contractions with at least three factors of the form $\nabla^{(m)} R_{a b c d}$. The terms indexed in $F$ are complete contractions in the form:

$$
\operatorname{contr}\left(\Delta^{q} \nabla^{\beta a} W_{a b c d} \otimes \Delta^{q^{\prime}} \nabla^{b \alpha} W_{\alpha \beta}^{c d}\right) .
$$

We will show below that by subtracting a divergence we can "get rid" of such complete contractions, modulo introducing correction terms with at least three factors.

Mini-proof of (2.13). This equation follows by an iterated application of the Leibniz rule ${ }^{21}$. Each derivative can hit either one of the curvature tensors $\tilde{R}_{i j k l}(\tilde{g})$, or one of the metric tensors $\tilde{g}^{a b}$ (with raised indices).

Now, we have sums $\sum_{x=\ldots}^{\ldots}$ in (2.13), to which we will refer to as the "first", "second" and "third" sum. Firstly, we observe that the expression $W_{i j k l} \Delta^{\frac{n}{2}-3} \nabla_{t}^{i} W^{t j k l}$ (along with its coefficient) arises when all $\frac{n}{2}-2$ derivatives $\partial_{\infty}$ hit precisely one of the two (ambient) curvature tensors - we then use the formula (2.9). Secondly, we observe that the first sum arises when all derivatives $\partial_{\infty}$ are forced to hit either of the two (ambient) curvature tensors, and moreover each curvature tensor must be hit by at least one derivative. Thirdly, the second sum arises when exactly one derivative $\partial_{\infty}$ hits one of the metric tensors $\tilde{g}^{A B}$ (recall that the only non-zero components of $\partial_{\infty} \tilde{g}^{a b}$ are $\partial_{\infty} \tilde{g}^{a b}=-2 P^{a b 22}$ and $\partial_{\infty} g^{\infty \infty}=-2$; notice that the first term may be discarded since it gives rise to terms with at least three factors). Fourthly, the third sum arises when exactly two derivatives $\partial_{\infty}$ hit metric terms $\tilde{g}^{A B}$ (a different term each).

\footnotetext{
${ }^{21}$ Recall that we think of $|\tilde{R}|_{\tilde{g}}^{2}$ as the product $\tilde{g}^{a \alpha} \tilde{g}^{b \beta} \tilde{g}^{c \gamma} \tilde{g}^{d \delta} \tilde{R}_{a b c d} \tilde{R}_{\alpha \beta \gamma \delta}$.

${ }^{22}$ When $1 \leq a, b \leq n$.
} 
Using (2.13) in conjunction with the formulas (2.5) and (2.7), we can derive (2.8). We can explicitly construct a divergence $\operatorname{div}_{i} T^{i}(g)$ so that for every $x$ :

$$
\begin{aligned}
& W_{i j k l} \Delta^{\frac{n}{2}-3} \nabla_{t}^{i} W^{t j k l}-\operatorname{div}_{i} T^{i}(g) \\
& =(-1)^{\frac{n}{2}} \nabla_{r_{1} \ldots r_{\frac{n}{2}-3}}^{\left(\frac{n}{2}-3\right)} \nabla^{s} W_{s j k l} \otimes\left(\nabla^{\left(\frac{n}{2}-3\right)}\right)^{r_{1} \ldots r_{\frac{n}{2}}-3} \nabla_{t} W^{t j k l}, \\
& \Delta^{x} \nabla^{s t} W_{s j t l} \otimes \Delta^{\frac{n}{2}-4-x} \nabla_{s^{\prime} t^{\prime}} W^{s^{\prime} j t^{\prime} l}-\operatorname{div}_{i} T^{i}(g) \\
& =(-1)^{\frac{n}{2}} \frac{1}{2} \nabla_{r_{1} \ldots r_{\frac{n}{2}-3}}^{\left(\frac{n}{2}-3\right)} \nabla^{s} W_{s j k l} \otimes\left(\nabla^{\left(\frac{n}{2}-3\right)}\right)^{r_{1} \ldots r \frac{n}{2}-3} \nabla_{t} W^{t j k l}, \\
& \Delta^{x} \nabla^{s} W_{s j k l} \otimes \Delta^{\frac{n}{2}-3-x} \nabla_{t} W^{t j k l}-\operatorname{div}_{i} T^{i}(g) \\
& =(-1)^{\frac{n}{2}-1} \nabla_{r_{1} \ldots r_{\frac{n}{2}-3}}^{\left(\frac{n}{2}-3\right)} \nabla^{s} W_{s j k l} \otimes\left(\nabla^{\left(\frac{n}{2}-3\right)}\right)^{r_{1} \ldots r_{\frac{n}{2}-3}} \nabla_{t} W^{t j k l}, \\
& \Delta^{x} \nabla^{a s} W_{s j k l} \otimes \Delta^{\frac{n}{2}-2-x} \nabla_{a t} W^{t j k l}-\operatorname{div}_{i} T^{i}(g) \\
& =(-1)^{\frac{n}{2}} \nabla_{r_{1} \ldots r \frac{n}{2}-3}^{\left(\frac{n}{2}-3\right)} \nabla^{s} W_{s j k l} \otimes\left(\nabla^{\left(\frac{n}{2}-3\right)}\right)^{r_{1} \ldots r_{\frac{n}{2}-3}} \nabla_{t} W^{t j k l}, \\
& \sum_{f \in F} a_{f} C^{f}(g)-\operatorname{div}_{i} T^{i}(g)=0 .
\end{aligned}
$$

All the above equations hold modulo complete contractions with at least three factors. We explain how the divergences for the above five equations are constructed. Pick out any Laplacian $\Delta\left(=\nabla_{a}^{a}\right),{ }^{23}$ appearing in any complete contraction above, and formally erase the upper index ${ }^{a}$. The resulting partial contraction is a Riemannian 1-tensor field with one free index ${ }_{a}$. Now, consider the divergence of this 1-tensor field and subtract it from the original complete contraction $^{24}$. The result (modulo correction terms with three factors that arise due to the curvature identity) is a new complete contraction with one fewer Laplacian. We iterate this step enough times, until in the end we obtain a complete contraction with no Laplacians. Observe that up to applying the curvature identity (and thus introducing correction terms with three factors), the resulting complete contraction is exactly the one claimed in (2.16), (2.17). In the other cases some additional divergences need to be subtracted. For (2.14) we consider the upper index $\nabla^{i}$ in the right factor; we erase this index (thus obtaining a Riemannian 1-tensor fields) and subtract the corresponding divergence. The result, up to applying the curvature identity, is the r.h.s. of (2.14). In the case of (2.15) we perform additional "integrations by parts" by erasing first the index ${ }^{s}$ in the first factor, and then the index $s^{\prime}$ in the second factor. The resulting complete contraction (after the curvature identity and formula (2.6) is the r.h.s. of (2.15). Finally, to derive (2.18) we integrate by parts the index ${ }^{b}$ in the expression

$$
\nabla_{t_{1} \ldots t_{q^{\prime}} r_{1} \ldots r_{q}}^{\left(q+q^{\prime}\right)} \nabla^{\beta a} W_{a b c d} \otimes \nabla^{\left(q+q^{\prime}\right)^{t_{1} \ldots t_{q^{\prime}} r_{1} \ldots r_{q}} \nabla^{b \alpha} W_{\alpha \beta c d}}
$$

the complete contraction we obtain will contain two differentiated Weyl tensors, one of which is in the form: $\nabla_{\ldots}^{b} \nabla^{a} W_{a b c d}$. This tensor vanishes, modulo a quadratic expression in curvatures, by virtue of the curvature identity. This concludes our proof of the above five equations.

Therefore, using the above formulas we derive that:

$$
\begin{aligned}
\partial_{\infty}^{\frac{n}{2}-2}\left(\left.\left|\tilde{R}_{i j k l}\right|\right|_{\tilde{g}} ^{2}\right)-\operatorname{div}_{i} T^{i}(g)=(-1)^{n}\left\{2 \cdot 2 \frac{1}{(n-3)(n-4) \cdots 4}\right. \\
\quad+2 \sum_{x=1}^{\frac{n}{2}-3}\left(\begin{array}{c}
\frac{n}{2}-2 \\
x
\end{array}\right)\left[\frac{1}{(n-3)(n-4) \cdots(n-2 x)}\right]\left[\frac{1}{(n-3)(n-4) \cdots(4+2 x)}\right]
\end{aligned}
$$

\footnotetext{
${ }^{23}$ A "Laplacian" here means two derivative indices that belong to the same factor and contract against each other.

${ }^{24}$ Further we refer to the subtraction of this explicitly constructed divergence as an "integration by parts".
} 


$$
\begin{aligned}
& -\sum_{x=0}^{\frac{n}{2}-3} 2 \cdot 4\left(\begin{array}{c}
\frac{n}{2}-2 \\
1
\end{array}\right) \cdot\left(\begin{array}{c}
\frac{n}{2}-3 \\
x
\end{array}\right) \cdot\left[\frac{1}{(n-3)(n-4) \cdots(n-2 x-2)}\right] \\
& \times\left[\frac{1}{(n-3)(n-4) \cdots(4+2 x)}\right]+\sum_{x=0}^{\frac{n}{2}-4} 2\left(\begin{array}{c}
\frac{n}{2}-2 \\
1
\end{array}\right) 2 \cdot 2 \cdot\left(\begin{array}{c}
\frac{n}{2}-3 \\
1
\end{array}\right) \cdot\left(\begin{array}{c}
\frac{n}{2}-4 \\
x
\end{array}\right) \\
& \left.\times\left[\frac{1}{(n-3)(n-4) \cdots(n-4-2 x)}\right] \cdot\left[\frac{1}{(n-3)(n-4) \cdot(4+2 x)}\right]\right\} \\
& \times \nabla_{r_{1} \ldots r_{\frac{n}{2}-3}}^{\left(\frac{n}{2}-3\right)} \nabla^{s} W_{s j k l}\left(\nabla^{\left(\frac{n}{2}-3\right)}\right)^{r_{1} \ldots r_{\frac{n}{2}-3}} \nabla_{t} W^{t j k l} .
\end{aligned}
$$

The above holds modulo terms of length at least 3 . Now, if we can show that the constant $\{\cdots\}$ is strictly positive, we will have shown (2.8).

We limit attention to the terms in the first sum with $x=y+2$, the terms in the second sum with $x=y+1$ and in the third sum with $x=y$ (for some given $y, 0 \leq y \leq \frac{n}{2}-4$ ). We observe that:

$$
\begin{aligned}
& 2\left(\begin{array}{c}
\frac{n}{2}-2 \\
y+2
\end{array}\right)\left[\frac{1}{(n-3)(n-4) \cdots(n-2(y+2))}\right]\left[\frac{1}{(n-3)(n-4) \cdots(4+2(y+2))}\right] \\
& -2 \cdot 4\left(\begin{array}{c}
\frac{n}{2}-2 \\
1
\end{array}\right) \cdot\left(\begin{array}{c}
\frac{n}{2}-3 \\
y+1
\end{array}\right) \cdot\left[\frac{1}{(n-3)(n-4) \cdots(n-2(y+1)-2)}\right] \\
& \times\left[\frac{1}{(n-3)(n-4) \cdots(4+2(y+1))}\right] \\
& +2\left(\begin{array}{c}
\frac{n}{2}-2 \\
1
\end{array}\right) 2 \cdot 2 \cdot\left(\begin{array}{c}
\frac{n}{2}-3 \\
1
\end{array}\right) \cdot\left(\begin{array}{c}
\frac{n}{2}-4 \\
y
\end{array}\right)\left[\frac{1}{(n-3)(n-4) \cdots(n-4-2 y)}\right] \\
& \times\left[\frac{1}{(n-3)(n-4) \cdot(4+2 y)}\right] \\
& =2 \cdot \frac{\left(\frac{n}{2}-2\right) \cdots\left(\frac{n}{2}-3-y\right)}{y !}\left[\frac{1}{(n-3)(n-4) \cdots(n-4-2 y)}\right] \\
& \times\left[\frac{1}{(n-3)(n-4) \cdots(4+2 y)}\right]\left\{\frac{(6+2 y)(4+2 y)}{(y+2)(y+1)}-4 \frac{4+2 y}{y+1}+4\right\}=0 .
\end{aligned}
$$

Now, the only terms we have not taken into account are the term $2 \cdot 2 \frac{2}{(n-3)(n-4) \cdots 4}$, the term in the first sum with $x=1$ and the term in the second sum with $x=0$. But we observe that those three terms add up to a positive number:

$$
\begin{aligned}
& 4 \frac{1}{(n-3)(n-4) \cdots 4}+2\left(\frac{n}{2}-2\right) \cdot \frac{1}{n-3} \frac{1}{(n-3)(n-4) \cdots 6} \\
& \quad-2 \cdot 4\left(\frac{n}{2}-2\right) \frac{1}{n-3} \frac{2}{(n-3)^{2}(n-4) \cdots 4}=4 \frac{1}{(n-3)(n-4) \cdots 4}>0 .
\end{aligned}
$$

Hence, using the above two formulas we derive that the constant in (2.19) is strictly positive and thus we derive our claim. This concludes the proof of Lemma 1.1.

\section{The proof of Lemmas 1.2, 1.3, 1.4, 1.5}

The three subsections below correspond to the cases $s<\sigma-2, s=\sigma-2, s=\sigma-1$. We also prove Lemma 1.5 in the subsections that deal with the cases $s=\sigma-2, s=\sigma-1$. 


\subsection{The proof of Lemmas 1.2, 1.3, 1.4 when $s<\sigma-2$}

Brief discussion. Recall the discussion regarding these lemmas in the introduction. Notice that since $s<\sigma-2$ we have $L^{*}=J^{*}=\varnothing$ by definition; thus in this case we only have to show Lemma 1.2. Therefore, we will show that there exists a linear combination of tensor fields, (indexed in $H$ below) so that:

$$
\sum_{f \in F^{*}} a_{f} C_{g}^{f}\left(\psi_{1}, \ldots, \phi_{s}\right)-\operatorname{div}_{i} \sum_{h \in H} a_{h} C_{g}^{h, i}\left(\psi_{1}, \ldots, \phi_{s}\right)=\sum_{f \in F^{O K}} a_{f} C_{g}^{f}\left(\psi_{1}, \ldots, \phi_{s}\right),
$$

where the tensor fields indexed in $F^{O K}$ have all the properties of the "cumbersome" tensor fields, but moreover have at most $\sigma-3$ factors in the form $R$ or $\Delta \psi_{h}$. That will prove Lemma 1.2 in this setting.

Rigorous discussion. We observe that the complete contractions in $I_{g}^{s}\left(\psi_{1}, \ldots, \psi_{s}\right)$ that have $|\Delta| \geq \sigma-2$ must be contractions $C^{f}$ indexed in $F^{\sigma-s} \cup F^{\sigma-s-1} \cup F^{\sigma-s-2}$. We index the complete contractions $C^{f}$ with $|\Delta|=\sigma-1$ in the sets $F_{|\Delta|=\sigma-1}^{\sigma-s}, F_{|\Delta|=\sigma-1}^{\sigma-s-1}, F_{|\Delta|=\sigma-1}^{\sigma-s-2}$ and the ones with $|\Delta|=\sigma-2$ in the sets $F_{|\Delta|=\sigma-2}^{\sigma-s}, F_{|\Delta|=\sigma-2}^{\sigma-s-1}, F_{|\Delta|=\sigma-2}^{\sigma-s-2}$, respectively. (Recall that the upper labels stand for the number of factors $\nabla^{(p)} \operatorname{Ric}$ or $R$ in the contractions indexed in $F$; the lower labels stand for the number of factors $R$ plus the number of factors $\Delta \psi_{h}$.) We claim:

Lemma 3.1. There is a linear combination of acceptable vector fields of length $\sigma$, say $\sum_{h \in H} a_{h} C_{g}^{h, i}\left(\psi_{1}, \ldots, \psi_{s}\right)$, so that in the notation above:

$$
\begin{aligned}
& \sum_{f \in F_{|\Delta|=\sigma-1}^{\sigma-s} \cup F_{|\Delta|=\sigma-1}^{\sigma-1} \cup F_{|\Delta|=\sigma-1}^{\sigma-s-2} \cup F_{|\Delta|=\sigma-2}^{\sigma-s} \cup F_{|\Delta|=\sigma-2}^{\sigma-s-1} \cup F_{|\Delta|=\sigma-2}^{\sigma-s-2}} a_{f} C_{g}^{f}\left(\psi_{1}, \ldots, \psi_{s}\right) \\
& -\operatorname{div}_{i} \sum_{h \in H} a_{h} C_{g}^{h, i}\left(\psi_{1}, \ldots, \psi_{s}\right)=\sum_{\substack{f \in F_{|\Delta| \leq \sigma-3}^{q>0, \delta>\mu} \\
|c|}} a_{f} C_{g}^{f}\left(\psi_{1}, \ldots, \psi_{s}\right),
\end{aligned}
$$

where the right hand side stands for a generic linear combination of acceptable complete contractions in the form (1.4) with $q>0$ factors $\nabla^{(p)} \operatorname{Ric}$ or $R$ and with $\delta>\mu$ and $|\Delta| \leq \sigma-3$.

We observe that if we can show the above, we will then clearly have proven the remaining case for our Lemma 1.2. So, the rest of this subsection is devoted to showing the above Lemma 3.1.

Proof of Lemma 3.1. We distinguish two cases and prove them separately. Either $\sigma<\frac{n}{2}-1$ or $\sigma=\frac{n}{2}-1$. We begin with the first case.

Proof of Lemma 3.1 in the case $\sigma<\frac{n}{2}-1$. The proof consists of three steps, which are spelled out along the proof.

We observe that each complete contraction in the form (1.4) with length $\sigma<\frac{n}{2}$ and weight $-n$ and $|\Delta|=\sigma-1$ (i.e. a total of $\sigma-1$ factors $\Delta \psi_{h}$ or $R$ ) must have the one factor that is not of the form $\Delta \psi_{h}$ or $R$ being in the form:

$$
\nabla^{a_{1} \ldots a_{\frac{n-2(\sigma-1)}{2}}} \nabla_{a_{1} \ldots a_{\frac{n-2(\sigma-1)}{2}}} \psi_{h}
$$

or in the form

$$
\nabla^{a_{1} \ldots a_{\frac{n-2(\sigma-1)}{2}}} \nabla_{a_{1} \ldots a_{\frac{n-2(\sigma-1)}{2}-2}} \operatorname{Ric}_{a_{\frac{n-2(\sigma-1)}{2}-1} a_{\frac{n-2(\sigma-1)}{2}}}
$$

Therefore all the complete contractions with $|\Delta|=\sigma-1$ must have $\delta \geq \mu+4$. 
First step in the proof of Lemma 3.1. Let $F_{\sigma-1}=F_{|\Delta|=\sigma-1}^{\sigma-s} \cup F_{|\Delta|=\sigma-1}^{\sigma-s-1} \cup F_{|\Delta|=\sigma-1}^{\sigma-s-2}$. We claim that there is a linear combination of vector fields in the form (1.4) with length $\sigma$, say $\sum_{h \in H} a_{h} C_{g}^{h, i}\left(\psi_{1}, \ldots, \psi_{s}\right)$ so that modulo complete contractions of length $\geq \sigma+1$ :

$$
\begin{aligned}
& \sum_{f \in F_{\sigma-1}} a_{f} C_{g}^{f}\left(\psi_{1}, \ldots, \psi_{s}\right)-\operatorname{div}_{i} \sum_{h \in H} a_{h} C_{g}^{h, i}\left(\psi_{1}, \ldots, \psi_{s}\right) \\
& \quad=\sum_{u=1}^{s}(\text { const })_{u} C_{g}^{u}\left(\psi_{1}, \ldots, \psi_{s}\right)+\sum_{w=1}^{s} \sum_{q=1}^{w-1}(\text { const })_{q, w} C_{g}^{w, q}\left(\psi_{1}, \ldots, \psi_{s}\right) \\
& \quad+(\text { const })_{*} C_{g}^{*}\left(\psi_{1}, \ldots, \psi_{s}\right)+\sum_{z \in Z} a_{z} C_{g}^{z}\left(\psi_{1}, \ldots, \psi_{s}\right)
\end{aligned}
$$

where $C_{g}^{*}\left(\psi_{1}, \ldots, \psi_{s}\right)$ stands for the complete contraction:

$$
\begin{aligned}
\operatorname{contr} & \left(\nabla^{a_{1} \ldots a_{\frac{n-2(\sigma-1)}{2}}-1} \nabla_{a_{1} \ldots a_{\frac{n-2(\sigma-1)}{2}-3}} \operatorname{Ric}_{a_{\frac{n-2(\sigma-2)}{2}-1} a_{\frac{n-2(\sigma-1)}{2}-1}}\right. \\
& \left.\otimes \nabla^{a b} \operatorname{Ric}_{a b} \otimes R^{\sigma-s-2} \otimes \Delta \psi_{1} \otimes \cdots \otimes \Delta \psi_{s}\right)
\end{aligned}
$$

whereas each $C_{g}^{u}\left(\psi_{1}, \ldots, \psi_{s}\right)$ stands for the complete contraction:

$$
\begin{aligned}
& \operatorname{contr}\left(\nabla^{a_{1} \ldots a_{\frac{n-2(\sigma-1)}{2}-1}} \nabla_{a_{1} \ldots a_{\frac{n-2(\sigma-1)}{2}-3}} \operatorname{Ric}_{a_{\frac{n-2(\sigma-2)}{2}-1} a_{\frac{n-2(\sigma-1)}{2}-1}} \otimes \nabla_{a b}^{a b} \psi_{u}\right. \\
& \left.\quad \otimes R^{\sigma-s-1} \otimes \Delta \psi_{1} \otimes \cdots \otimes \Delta \hat{\psi_{u}} \otimes \Delta \psi_{s}\right)
\end{aligned}
$$

and each $C_{g}^{w, q}\left(\psi_{1}, \ldots, \psi_{s}\right)$ stands for the complete contraction:

$$
\begin{aligned}
\operatorname{contr} & \left(\nabla^{a_{1} \ldots a_{\frac{n-2(\sigma-1)}{2}-1}} \nabla_{a_{1} \ldots a_{\frac{n-2(\sigma-1)}{2}-1}} \psi_{w} \otimes \nabla_{a b}^{a b} \psi_{q}\right. \\
& \left.\otimes R^{\sigma-s} \otimes \Delta \psi_{1} \otimes \cdots \otimes \Delta \hat{\psi_{q}} \otimes \cdots \otimes \Delta \hat{\psi}_{w} \otimes \cdots \otimes \Delta \psi_{s}\right) .
\end{aligned}
$$

Finally, $\sum_{z \in Z} a_{z} C_{g}^{z}\left(\psi_{1}, \ldots, \psi_{s}\right)$ stands for a generic linear combination of complete contractions in the form (1.4) with $|\Delta| \leq \sigma-3$ and $\delta \geq \mu+2$. Moreover, we claim that each of the complete contractions $C^{*}, C^{w, q}, C^{u}$ above has $\delta \geq \mu+4$.

Proof of (3.4). We present the proof of this claim in detail, as the same argument will be used repeatedly in many other instances. We have already observed that we can write out:

$$
\sum_{f \in F_{\sigma-1}} a_{f} C_{g}^{f}\left(\psi_{1}, \ldots, \psi_{s}\right)=(\text { const }){ }_{1} C_{g}^{I}\left(\psi_{1}, \ldots, \psi_{s}\right)+\sum_{y=1}^{s}(\text { const })_{y} C_{g}^{I I, y}\left(\psi_{1}, \ldots, \psi_{s}\right) .
$$

Here $C^{I}$ is the complete contraction with one factor as in (3.3) and $\sigma-s-1$ factors $R$ and $s$ factors $\Delta \psi_{1}, \ldots, \Delta \psi_{s} . C^{I I, y}$ is the complete contraction with one factor as in (3.2) and $\sigma-s$ factors $R$ and $s-1$ factors $\Delta \psi_{1}, \ldots, \Delta \hat{\psi}_{y}, \ldots \Delta \psi_{s}$. In particular, $C^{I}$ is in the form:

$$
\begin{aligned}
\operatorname{contr} & \left(\nabla^{a_{1} \ldots a_{\frac{n-2(\sigma-1)}{2}}} \nabla_{a_{1} \ldots a_{\frac{n-2(\sigma-1)}{2}-2}} \operatorname{Ric}_{a_{\frac{n-2(\sigma-1)}{2}-1} a_{\frac{n-2(\sigma-1)}{2}}}\right. \\
& \left.\otimes R^{\sigma-s-1} \otimes \Delta \psi_{1} \otimes \cdots \otimes \Delta \psi_{s}\right) .
\end{aligned}
$$

Now, if we can show that we can find vector fields

$$
\sum_{h \in H_{1}} a_{h} C_{g}^{h, i}\left(\psi_{1}, \ldots, \psi_{s}\right), \quad \sum_{h \in H_{2}} a_{h} C_{g}^{h, i}\left(\psi_{1}, \ldots, \psi_{s}\right),
$$


so that:

$$
\begin{aligned}
& C_{g}^{I}\left(\psi_{1}, \ldots, \psi_{s}\right)-\operatorname{div}_{i} \sum_{h \in H_{1}} a_{h} C_{g}^{h, i}\left(\psi_{1}, \ldots, \psi_{s}\right) \\
& =\sum_{u=1}^{s}(\text { const })_{u} C_{g}^{u}\left(\psi_{1}, \ldots, \psi_{s}\right)+\sum_{w=1}^{s} \sum_{q=1}^{w-1}(\text { const })_{q, w} C_{g}^{w, q}\left(\psi_{1}, \ldots, \psi_{s}\right) \\
& \quad+(\text { const })_{*} C_{g}^{*}\left(\psi_{1}, \ldots, \psi_{s}\right)+\sum_{z \in Z} a_{z} C_{g}^{z}\left(\psi_{1}, \ldots, \psi_{s}\right)
\end{aligned}
$$

and

$$
\begin{aligned}
C_{g}^{I I, y} & \left(\psi_{1}, \ldots, \psi_{s}\right)-\operatorname{div}_{i} \sum_{h \in H_{2}} a_{h} C_{g}^{h, i}\left(\psi_{1}, \ldots, \psi_{s}\right) \\
& =\sum_{u=1}^{s}(\text { const })_{u} C_{g}^{u}\left(\psi_{1}, \ldots, \psi_{s}\right)+\sum_{w=1}^{s} \sum_{q=1}^{w-1}(\text { const })_{q, w} C_{g}^{w, q}\left(\psi_{1}, \ldots, \psi_{s}\right) \\
& +(\text { const })_{*} C_{g}^{*}\left(\psi_{1}, \ldots, \psi_{s}\right)+\sum_{z \in Z} a_{z} C_{g}^{z}\left(\psi_{1}, \ldots, \psi_{s}\right),
\end{aligned}
$$

then clearly (3.4) will follow. We first prove (3.6).

Proof of (3.6). We define $C_{g}^{I, a_{1}}\left(\psi_{1}, \ldots, \psi_{s}\right)$ to stand for the vector field that arises from $C^{I}$ by erasing the index ${ }^{a_{1}}$ in the first factor (see (3.5)) and making the index $a_{1}$ that is then left into a free index. We then calculate:

$$
\begin{aligned}
& C_{g}^{I}\left(\psi_{1}, \ldots, \psi_{s}\right)-\operatorname{div}_{a_{1}} C_{g}^{I, a_{1}}\left(\psi_{1}, \ldots, \psi_{s}\right) \\
& \quad=-(\sigma-s-1) C_{g}^{I, A}\left(\psi_{1}, \ldots, \psi_{s}\right)-\sum_{h=1}^{s} C_{g}^{I, h}\left(\psi_{1}, \ldots, \psi_{s}\right),
\end{aligned}
$$

where $C^{I, A}$ stands for the complete contraction that arises in $\operatorname{div}_{a_{1}} C_{g}^{I, a_{1}}\left(\psi_{1}, \ldots, \psi_{s}\right)$ when $\nabla_{a_{1}}$ hits one of the factors $R$ and $C^{I, h}$ stands for the complete contraction that arises when $\nabla_{a_{1}}$ hits the factor $\Delta \psi_{h}$. Given $C^{I, A}$, we then define $C_{g}^{I, A, a_{1}}\left(\psi_{1}, \ldots, \psi_{s}\right)$ to stand for the vector field that arises by erasing the index $a_{1}$ in its first factor (note it is a derivative index) and making the index $a_{1}$ in the factor $\nabla_{a_{1}} R$ into a free index. Also, for each $h, 1 \leq h \leq s$, we define $C_{g}^{I, h, a_{1}}$ to stand for the vector field that arises from $C_{g}^{I, h}\left(\psi_{1}, \ldots, \psi_{s}\right)$ by erasing the index $a_{1}$ in its first factor (note it is a derivative index) and making the index $a_{1}$ in the factor $\nabla_{a_{1}} \Delta \psi_{h}$ into a free index. We then calculate:

$$
\begin{aligned}
& {\left[C_{g}^{I}\left(\psi_{1}, \ldots, \psi_{s}\right)-\operatorname{div}_{a_{1}} C_{g}^{I, a_{1}}\left(\psi_{1}, \ldots, \psi_{s}\right)\right]} \\
& \quad-(\sigma-s-1) \operatorname{div}_{a_{1}} C_{g}^{I, A, a_{1}}\left(\psi_{1}, \ldots, \psi_{s}\right)-\operatorname{div}_{a_{1}} \sum_{h=1}^{s} C_{g}^{I, h, a_{1}}\left(\psi_{1}, \ldots, \psi_{s}\right) \\
& \quad=(\sigma-s-1) C_{g}^{*}\left(\psi_{1}, \ldots, \psi_{s}\right)+\sum_{u=1}^{s} C_{g}^{u}\left(\psi_{1}, \ldots, \psi_{s}\right)+\sum_{z \in Z} a_{z} C_{g}^{z}\left(\psi_{1}, \ldots, \psi_{s}\right) .
\end{aligned}
$$

(We are using the notation of (3.4).) (3.8) just follows by the definitions. This proves (3.6).

Proof of (3.7). We denote by $C_{g}^{I I, y, *}\left(\psi_{1}, \ldots, \psi_{s}\right)$ the complete contraction:

$$
\operatorname{contr}\left(\Delta^{\frac{n-2(\sigma-1)}{2}-1} \psi_{y} \otimes \Delta R \otimes R^{\sigma-s-1} \otimes \Delta \psi_{1} \otimes \cdots \otimes \Delta \hat{\psi_{y}} \otimes \cdots \otimes \Delta \psi_{s}\right) .
$$


Then, by complete analogy with the previous case, we explicitly construct a divergence, $\operatorname{div}_{i} \sum_{h \in H} a_{h} C_{g}^{h, i}\left(\psi_{1}, \ldots, \psi_{s}\right)$ from $C_{g}^{I I, y}\left(\psi_{1}, \ldots, \psi_{s}\right)$ so that:

$$
\begin{aligned}
C_{g}^{I I, y} & \left(\psi_{1}, \ldots, \psi_{s}\right)-\operatorname{div}_{i} \sum_{h \in H} a_{h} C_{g}^{h, i}\left(\psi_{1}, \ldots, \psi_{s}\right) \\
& =\sum_{y^{\prime} \neq y} C_{g}^{y, y^{\prime}}\left(\psi_{1}, \ldots, \psi_{s}\right)+C_{g}^{I I, y, *}\left(\psi_{1}, \ldots, \psi_{s}\right)+\sum_{z \in Z} a_{z} C_{g}^{z}\left(\psi_{1}, \ldots, \psi_{s}\right) .
\end{aligned}
$$

Now, clearly $C_{g}^{I I, y, *}\left(\psi_{1}, \ldots, \psi_{s}\right)$ is not in the form $C^{u}$ or $C^{*}$ above. Also, for each of the complete contractions $C_{g}^{y, y^{\prime}}\left(\psi_{1}, \ldots, \psi_{s}\right)$, we inquire whether $y>y^{\prime}$ or $y<y^{\prime}$. In the first case, we actually have a complete contraction in the form $C^{q, w}$ that is allowed in the right hand side of (3.7). So in that case, we keep the complete contraction $C^{y, y^{\prime}}$.

In the second case, $C^{y, y^{\prime}}$ is not a complete contraction in one of the forms on the right hand side of (3.7). Now, by repeating the same argument as before, it follows that we can explicitly construct a divergence $\operatorname{div}_{i} \sum_{h \in H} a_{h} C_{g}^{h, i}\left(\psi_{1}, \ldots, \psi_{s}\right)$ such that $y<y^{\prime}$ and deduce that modulo complete contractions of length $\geq \sigma+1$ :

$$
\begin{array}{r}
C_{g}^{y, y^{\prime}}\left(\psi_{1}, \ldots, \psi_{s}\right)-\operatorname{div}_{i} \sum_{h \in H} a_{h} C_{g}^{h, i}\left(\psi_{1}, \ldots, \psi_{s}\right) \\
=C_{g}^{y^{\prime}, y}\left(\psi_{1}, \ldots, \psi_{s}\right)+\sum_{z \in Z} a_{z} C_{g}^{z}\left(\psi_{1}, \ldots, \psi_{s}\right), \\
C_{g}^{I I, y, *}\left(\psi_{1}, \ldots, \psi_{s}\right)-\operatorname{div}_{i} \sum_{h \in H} a_{h} C_{g}^{h, i}\left(\psi_{1}, \ldots, \psi_{s}\right) \\
=C_{g}^{y, *}\left(\psi_{1}, \ldots, \psi_{s}\right)+\sum_{z \in Z} a_{z} C_{g}^{z}\left(\psi_{1}, \ldots, \psi_{s}\right) .
\end{array}
$$

Thus, we have shown (3.7) and therefore (3.4).

A study of the complete contractions with $|\Delta|=\sigma-2$ in (3.1). Now, we focus on the complete contractions in the index sets $F^{q}, q>0$ in (3.1) that have $|\Delta|=\sigma-2$. We have observed that only complete contractions in $F^{\sigma-s}, F^{\sigma-s-1}$ and $F^{\sigma-s-2}$ can have $|\Delta|=$ $\sigma-2$. We have denoted the respective index sets by $F_{|\Delta|=\sigma-2}^{\sigma-s} \subset F^{\sigma-s}, F_{|\Delta|=\sigma-2}^{\sigma-s-1} \subset F^{\sigma-s-1}$ and $F_{|\Delta|=\sigma-2}^{\sigma-s-2} \subset F^{\sigma-s-2}$. Clearly, since we are dealing with the case $s<\sigma-2$, each complete contraction $C^{f}$ indexed in one of the three sets above must have at least one factor $R$. Hence (by the "Important remark" in the introduction) it follows that each complete contraction $C^{f}$ above has $\delta \geq \mu+2$. Therefore, by the definition of $F_{|\Delta|=\sigma-2}^{\sigma-s}$, we derive that we can write out:

$$
\begin{aligned}
& \sum_{\substack{f \in F_{|\Delta|=\sigma-2}^{\sigma-s}\\
}} a_{f} C_{g}^{f}\left(\psi_{1}, \ldots, \psi_{s}\right)=\sum_{\substack{f \in F_{|\Delta|=\sigma-2, A}^{\sigma-s}\\
}} a_{f} C_{g}^{f}\left(\psi_{1}, \ldots, \psi_{s}\right) \\
& \quad+\sum_{\substack{f \in F_{|\Delta|=\sigma-2, B}^{\sigma-s}\\
}} a_{f} C_{g}^{f}\left(\psi_{1}, \ldots, \psi_{s}\right)+\sum_{\substack{f \in F_{|\Delta|=\sigma-2, C}^{\sigma-s} \\
a_{f}}} a_{f} C_{g}^{f}\left(\psi_{1}, \ldots, \psi_{s}\right) .
\end{aligned}
$$

Here $\sum_{f \in F_{|\Delta|=\sigma-2, A}^{\sigma-s}} a_{f} C_{g}^{f}\left(\psi_{1}, \ldots, \psi_{s}\right)$ stands for a linear combination of complete contractions in the form:

$$
\operatorname{contr}\left(\nabla^{a_{1} \ldots a_{t}} \nabla_{r_{1} \ldots r_{p}}^{(p)} \operatorname{Ric}_{i j} \otimes \nabla^{b_{1} \ldots b_{s}} \nabla_{z_{1} \ldots z_{q}}^{(q)} \operatorname{Ric}_{i^{\prime} j^{\prime}} \otimes R^{\sigma-s-2} \otimes \Delta \psi_{1} \otimes \cdots \otimes \Delta \psi_{s}\right),
$$

with $\delta \geq \mu+2$ (we observe that all the indices $r_{1}, \ldots, r_{p},{ }_{i},{ }_{j}$ in the first factor that are not involved in an internal contraction must contract against an index $z_{1}, \ldots, z_{q}, i^{\prime}, j^{\prime}$ in the second 
factor and vice versa). $\sum_{f \in F_{|\Delta|=\sigma-2, B}^{\sigma-s}} a_{f} C_{g}^{f}\left(\psi_{1}, \ldots, \psi_{s}\right)$ stands for a generic linear combination of complete contractions in the form:

$$
\begin{aligned}
& \operatorname{contr}\left(\nabla^{a_{1} \ldots a_{t}} \nabla_{r_{1} \ldots r_{p}}^{(p)} \operatorname{Ric}_{i j} \otimes \nabla^{b_{1} \ldots b_{y}} \nabla_{z_{1} \ldots z_{q}}^{(q)} \psi_{h} \otimes R^{\sigma-s-2}\right. \\
& \left.\otimes \Delta \psi_{1} \otimes \cdots \otimes \Delta \hat{\psi_{h}} \otimes \cdots \otimes \Delta \psi_{s}\right)
\end{aligned}
$$

with $\delta \geq \mu+2$ and where $y+q \geq 2$ (but the second factor is not in the form $\Delta \psi_{h}$, by definition) and where as above all the indices $r_{1}, \ldots, r_{p},{ }_{i},{ }_{j}$ in the first factor that are not involved in an internal contraction must contract against an index $z_{1}, \ldots, z_{q}$ in the second factor and vice versa.

Finally, $\sum_{f \in F_{|\Delta|=\sigma-2, C}^{\sigma-s}} a_{f} C_{g}^{f}\left(\psi_{1}, \ldots, \psi_{s}\right)$ stands for a generic linear combination of complete contractions in the form:

$$
\begin{array}{r}
\operatorname{contr}\left(\nabla^{a_{1} \ldots a_{t}} \nabla_{r_{1} \ldots r_{p}}^{(p)} \psi_{h} \otimes \nabla^{b_{1} \ldots b_{y}} \nabla_{z_{1} \ldots z_{q}}^{(q)} \psi_{h^{\prime}} \otimes R^{\sigma-s-2}\right. \\
\left.\otimes \Delta \psi_{1} \otimes \cdots \otimes \Delta \hat{\psi_{h}} \otimes \cdots \otimes \Delta \hat{\psi}_{h^{\prime}} \otimes \cdots \otimes \Delta \psi_{s}\right),
\end{array}
$$

with $\delta \geq \mu+2$ and where $t+p, y+q \geq 2$ (and neither of the first two factors is of the form $\Delta \psi_{b}$ ) and all the indices $r_{1}, \ldots, r_{p}$ in the first factor that are not involved in an internal contraction must contract against an index $z_{1}, \ldots, z_{q}$ in the second factor and vice versa.

Similarly, we see that we can write out:

$$
\sum_{f \in F_{|\Delta|=\sigma-2}^{\sigma-s-1}} a_{f} C_{g}^{f}\left(\psi_{1}, \ldots, \psi_{s}\right)=\sum_{f \in F_{|\Delta|=\sigma-2, \alpha}^{\sigma-s-1}} a_{f} C_{g}^{f}\left(\psi_{1}, \ldots, \psi_{s}\right)+\sum_{f \in F_{|\Delta|=\sigma-2, \beta}^{\sigma-s-1}} a_{f} C_{g}^{f}\left(\psi_{1}, \ldots, \psi_{s}\right),
$$

where $\sum_{f \in F_{|\Delta|=\sigma-2, \alpha}^{\sigma-s-1}} a_{f} C_{g}^{f}\left(\psi_{1}, \ldots, \psi_{s}\right)$ stands for a generic linear combination of complete contractions in the form:

$$
\begin{aligned}
& \operatorname{contr}\left(\nabla^{a_{1} \ldots a_{t}} \nabla_{r_{1} \ldots r_{m}}^{(m)} R_{i j k l} \otimes \nabla^{b_{1} \ldots b_{s}} \nabla_{z_{1} \ldots z_{q}}^{(q)} \psi_{h} \otimes R^{\sigma-s-1}\right. \\
& \left.\otimes \Delta \psi_{1} \otimes \cdots \otimes \Delta \hat{\psi}_{h} \otimes \cdots \otimes \Delta \psi_{s}\right),
\end{aligned}
$$

with $\delta \geq \mu+2$ and where $s+q \geq 2$ and where as above we observe that all the indices $r_{1}, \ldots, r_{m},{ }_{i},{ }_{j}, k, l$ in the first factor that are not involved in an internal contraction must contract against an index $z_{1}, \ldots, z_{q}$ in the second factor and vice versa.

Also, $\sum_{f \in F_{|\Delta|=\sigma-2, \beta}^{\sigma-s-1}} a_{f} C_{g}^{f}\left(\psi_{1}, \ldots, \psi_{s}\right)$ stands for a generic linear combination of complete contractions in the form:

$$
\operatorname{contr}\left(\nabla^{a_{1} \ldots a_{t}} \nabla_{r_{1} \ldots r_{m}}^{(m)} R_{i j k l} \otimes \nabla^{b_{1} \ldots b_{s}} \nabla_{z_{1} \ldots z_{q}}^{(q)} \operatorname{Ric}_{i^{\prime} j^{\prime}} \otimes R^{\sigma-s-2} \otimes \Delta \psi_{1} \otimes \cdots \otimes \Delta \psi_{s}\right),
$$

with $\delta \geq \mu+2$ and where as above we observe that all the indices $r_{1}, \ldots, r_{p},{ }_{i},{ }_{j},{ }_{k}, l$ in the first factor that are not involved in an internal contraction must contract against an index $z_{1}, \ldots, z_{q}, i^{\prime}, j^{\prime}$ in the second factor and vice versa.

Finally, it follows that:

$$
\sum_{\substack{f \in F_{|\Delta|=\sigma-2}^{\sigma-s-2} \\ \mid}} a_{f} C_{g}^{f}\left(\psi_{1}, \ldots, \psi_{s}\right)=\sum_{\substack{f \in F_{|\Delta|=\sigma-2, \gamma}^{\sigma-s-2}\\}} a_{f} C_{g}^{f}\left(\psi_{1}, \ldots, \psi_{s}\right)
$$

where $\sum_{f \in F_{|\Delta|=\sigma-2, \gamma}^{\sigma-s-2}} a_{f} C_{g}^{f}\left(\psi_{1}, \ldots, \psi_{s}\right)$ stands for a linear combination of complete contractions in the form:

$$
\operatorname{contr}\left(\nabla^{a_{1} \ldots a_{t}} \nabla_{r_{1} \ldots r_{p}}^{(p)} R_{i j k l} \otimes \nabla^{b_{1} \ldots b_{s}} \nabla_{z_{1} \ldots z_{q}}^{(q)} R_{i^{\prime} j^{\prime} k^{\prime} l^{\prime}} \otimes R^{\sigma-s-2} \otimes \Delta \psi_{1} \otimes \cdots \otimes \Delta \psi_{s}\right)
$$


(all the indices $r_{1}, \ldots, r_{p}, i, j,,_{k}, l$ in the first factor that are not involved in an internal contraction must contract against an index $z_{1}, \ldots, z_{q}, i^{\prime}, j^{\prime}, k^{\prime}, l^{\prime}$ in the second factor and vice versa).

Second step in the proof of Lemma 3.1. The second step consists of proving the equations (3.15), (3.16), (3.17), (3.18), (3.19), (3.20), (3.21) below. We firstly claim that we can subtract a divergence, $\operatorname{div}_{i} \sum_{h \in H} a_{h} C_{g}^{h, i}\left(\psi_{1}, \ldots, \psi_{s}\right)$, from the sublinear combination $\sum_{f \in F_{|\Delta|=\sigma-2, A}^{\sigma-s}} a_{f} C_{g}^{f}\left(\psi_{1}, \ldots, \psi_{s}\right)$ so as to obtain an equation, modulo complete contractions of length $\geq \sigma+1$ :

$$
\begin{aligned}
& \sum_{\substack{f \in F_{|\Delta|=\sigma-2, A}^{\sigma-s}\\
}} a_{f} C_{g}^{f}\left(\psi_{1}, \ldots, \psi_{s}\right)-\operatorname{div}_{i} \sum_{h \in H} a_{h} C_{g}^{h, i}\left(\psi_{1}, \ldots, \psi_{s}\right) \\
& \quad=(\mathrm{const})_{1} \cdot C_{g}^{*}\left(\psi_{1}, \ldots, \psi_{s}\right)+\sum_{f \in F_{|\Delta|=\sigma-2, \beta}^{\sigma-s-1}} a_{f} C_{g}^{f}\left(\psi_{1}, \ldots, \psi_{s}\right)+\sum_{z \in Z^{\prime}} a_{z} C_{g}^{z}\left(\psi_{1}, \ldots, \psi_{s}\right)
\end{aligned}
$$

where $C_{g}^{*}\left(\psi_{1}, \ldots, \psi_{s}\right)$ stands (as before) for the complete contraction:

$$
\operatorname{contr}\left(\nabla^{a_{1} \ldots a_{\frac{n}{2}-4} i j} \nabla_{a_{1} \ldots a \frac{n}{2}-4}^{\left(\frac{n}{2}-4\right)} \operatorname{Ric}_{i j} \otimes \nabla^{i^{\prime} j^{\prime}} \operatorname{Ric}_{i^{\prime} j^{\prime}} \otimes R^{\sigma-s-2} \otimes \Delta \psi_{1} \otimes \cdots \otimes \Delta \psi_{s}\right)
$$

while $\sum_{f \in F_{|\Delta|=\sigma-2, \beta}^{\sigma-s-1}} a_{f} C_{g}^{f}\left(\psi_{1}, \ldots, \psi_{s}\right)$ again stands for a generic linear combination of complete contractions in the form (3.13) and $\sum_{z \in Z^{\prime}} a_{z} C_{g}^{z}\left(\psi_{1}, \ldots, \psi_{s}\right)$ stands for a generic linear combination of complete contractions in the form (1.4) with length $\sigma, \delta \geq \mu+1$ and $|\Delta|=\sigma-3$.

Next, consider the linear combination $\sum_{f \in F_{|\Delta|=\sigma-2, B}^{\sigma-s}} a_{f} C_{g}^{f}\left(\psi_{1}, \ldots, \psi_{s}\right)$. We then claim that there is a divergence of a vector field, $\operatorname{div}_{i} \sum_{h \in H} a_{h} C_{g}^{h, i}\left(\psi_{1}, \ldots, \psi_{s}\right)$, so that, modulo complete contractions of length $\geq \sigma+1$ :

$$
\begin{aligned}
& \sum_{\substack{f \in F_{|\Delta|=\sigma-2, B}^{\sigma-s}\\
}} a_{f} C_{g}^{f}\left(\psi_{1}, \ldots, \psi_{s}\right)-\operatorname{div}_{i} \sum_{h \in H} a_{h} C_{g}^{h, i}\left(\psi_{1}, \ldots, \psi_{s}\right) \\
& \quad=\sum_{y=1}^{s}(\mathrm{const})_{y} \cdot C_{g}^{y}\left(\psi_{1}, \ldots, \psi_{s}\right)+\sum_{\substack{f \in F_{|\Delta|=\sigma-2, \alpha}^{\sigma-s-1} \\
\mid=0}} a_{f} C_{g}^{f}\left(\psi_{1}, \ldots, \psi_{s}\right)+\sum_{z \in Z^{\prime}} a_{z} C_{g}^{z}\left(\psi_{1}, \ldots, \psi_{s}\right),
\end{aligned}
$$

where the complete contractions $C_{g}^{y}\left(\psi_{1}, \ldots, \psi_{s}\right)$ are in the form (3.11) with every index involved in an internal contraction, and the linear combination $\sum_{z \in Z^{\prime}} a_{z} C_{g}^{z}\left(\psi_{1}, \ldots, \psi_{s}\right)$ is the same as in $(3.15)$.

Finally, we consider the linear combination $\sum_{f \in F_{|\Delta|=\sigma-2, C}^{\sigma-s}} a_{f} C_{g}^{f}\left(\psi_{1}, \ldots, \psi_{s}\right)$. Just by switching two factors, we assume that $h>h^{\prime}$ in (3.12). We then claim that we can construct a divergence of a vector field, $\operatorname{div}_{i} \sum_{h \in H} a_{h} C_{g}^{h, i}\left(\psi_{1}, \ldots, \psi_{s}\right)$, so that, modulo complete contractions of length $\geq \sigma+1$ :

$$
\begin{aligned}
& \sum_{\substack{f \in F_{|\Delta|=\sigma-2, C}^{\sigma-s} \\
\mid s}} a_{f} C_{g}^{f}\left(\psi_{1}, \ldots, \psi_{s}\right)-\operatorname{div}_{i} \sum_{h \in H} a_{h} C_{g}^{h, i}\left(\psi_{1}, \ldots, \psi_{s}\right) \\
& \quad=\sum_{h=1}^{s} \sum_{h^{\prime}=1}^{h-1}(\text { const })_{h, h^{\prime}} \cdot C_{g}^{h, h^{\prime}}\left(\psi_{1}, \ldots, \psi_{s}\right)+\sum_{z \in Z^{\prime}} a_{z} C_{g}^{z}\left(\psi_{1}, \ldots, \psi_{s}\right),
\end{aligned}
$$

where the complete contractions $C_{g}^{h, h^{\prime}}\left(\psi_{1}, \ldots, \psi_{s}\right)$ are in the form (3.12) with every index involved in an internal contraction and the linear combination $\sum_{z \in Z^{\prime}} a_{z} C_{g}^{z}\left(\psi_{1}, \ldots, \psi_{s}\right)$ is the same as above. 
More claims. We now consider the generic linear combinations $\sum_{f \in F_{|\Delta|=\sigma-\alpha, \alpha}^{\sigma-s-1}} a_{f} C_{g}^{f}\left(\psi_{1}, \ldots, \psi_{s}\right)$, $\sum_{f \in F_{|\Delta|=\sigma-2, \beta}^{\sigma-s-1}} a_{f} C_{g}^{f}\left(\psi_{1}, \ldots, \psi_{s}\right)$. In order to state our next claims, we define two complete contractions: $C_{g}^{+}\left(\psi_{1}, \ldots, \psi_{s}\right)$ will stand for the complete contraction:

$$
\operatorname{contr}\left(\nabla^{a_{1} \ldots a_{\frac{n-2 \sigma}{2}-1}^{i l}} \nabla_{a_{1} \ldots a_{\frac{n-2 \sigma}{2}-1}} R_{i j k l} \otimes \operatorname{Ric}^{j k} \otimes R^{\sigma-s-2} \otimes \Delta \psi_{1} \otimes \cdots \otimes \Delta \psi_{s}\right),
$$

and $C_{g}^{++, h}\left(\psi_{1}, \ldots, \psi_{s}\right), 1 \leq h \leq s$ will stand for the complete contraction:

$$
\begin{aligned}
& \operatorname{contr}\left(\nabla^{a_{1} \ldots a_{\frac{n-2 \sigma}{2}-1} i l} \nabla_{a_{1} \ldots a_{\frac{n-2 \sigma}{2}-1}} R_{i j k l} \otimes \nabla^{j k} \psi_{h} \otimes R^{\sigma-s-1}\right. \\
& \left.\otimes \Delta \psi_{1} \otimes \cdots \otimes \Delta \hat{\psi}_{h} \otimes \cdots \otimes \Delta \psi_{s}\right) .
\end{aligned}
$$

Moreover, we define $C_{g}^{\sharp}\left(\psi_{1}, \ldots, \psi_{s}\right)$ to stand for the complete contraction:

$$
\operatorname{contr}\left(\nabla^{a_{1} \ldots a_{\frac{n-2 \sigma}{2}-3}{ }^{i l}} \nabla_{a_{1} \ldots a_{\frac{n-2 \sigma}{2}-3}} R_{i j k l} \otimes \nabla^{a d} R_{a}{ }^{j k}{ }_{d} \otimes R^{\sigma-s-1} \otimes \Delta \psi_{1} \otimes \cdots \otimes \Delta \psi_{s}\right) .
$$

Next claims. Consider the linear combination $\sum_{f \in F_{|\Delta|=\sigma-2, \alpha}^{\sigma-s-1}} a_{f} C_{g}^{f}\left(\psi_{1}, \ldots, \psi_{s}\right)$. We claim that there is a divergence of a vector field, $\operatorname{div}_{i} \sum_{h \in H} a_{h} C_{g}^{h, i}\left(\psi_{1}, \ldots, \psi_{s}\right)$, so that, modulo complete contractions of length $\geq \sigma+1$ :

$$
\begin{aligned}
& \sum_{\substack{\sigma \in F_{|\Delta|=\sigma-2, \alpha}^{\sigma-s-1} \\
\mid s}} a_{f} C_{g}^{f}\left(\psi_{1}, \ldots, \psi_{s}\right)-\operatorname{div}_{i} \sum_{h \in H} a_{h} C_{g}^{h, i}\left(\psi_{1}, \ldots, \psi_{s}\right) \\
& \quad=\sum_{y=1}^{s}(\text { const })_{y}^{\prime} \cdot C_{g}^{++, y}\left(\psi_{1}, \ldots, \psi_{s}\right)+\sum_{z \in Z^{\prime}} a_{z} C_{g}^{z}\left(\psi_{1}, \ldots, \psi_{s}\right),
\end{aligned}
$$

and furthermore, for each $C^{++, y}$, we have $\delta\left(C^{++, h}\right) \geq \mu+2$.

On the other hand, we consider the linear combination $\sum_{f \in F_{|\Delta|=\sigma-2, \beta}^{\sigma-s}} a_{f} C_{g}^{f}\left(\psi_{1}, \ldots, \psi_{s}\right)$. We then claim that there is a divergence of a vector field, $\operatorname{div}_{i} \sum_{h \in H} a_{h} C_{g}^{h, i}\left(\psi_{1}, \ldots, \psi_{s}\right)$, so that, modulo complete contractions of length $\geq \sigma+1$ :

$$
\begin{aligned}
& \sum_{\substack{f \in F_{|\Delta|=\sigma-2, \beta}^{\sigma-s-1} \\
\mid \Delta-\sigma,}} a_{f} C_{g}^{f}\left(\psi_{1}, \ldots, \psi_{s}\right)-\operatorname{div}_{i} \sum_{h \in H} a_{h} C_{g}^{h, i}\left(\psi_{1}, \ldots, \psi_{s}\right) \\
& \quad=\sum_{f \in F_{|\Delta|=\sigma-2, \gamma}^{\sigma-s-1}} a_{f} C_{g}^{f}\left(\psi_{1}, \ldots, \psi_{s}\right)+(\mathrm{const})^{\prime \prime} \cdot C_{g}^{+}\left(\psi_{1}, \ldots, \psi_{s}\right)+\sum_{z \in Z^{\prime}} a_{z} C_{g}^{z}\left(\psi_{1}, \ldots, \psi_{s}\right)
\end{aligned}
$$

and furthermore for $C^{+}$, we have $\delta\left(C^{+}\right) \geq \mu+2$. (For the definition of $\sum_{f \in F_{|\Delta|=\sigma-2, \gamma}^{\sigma-s-1}} \cdots$, see (3.14).) that:

Finally, we claim that there are divergences of vector fields, $\operatorname{div}_{i} \sum_{h \in H} a_{h} C_{g}^{h, i}\left(\psi_{1}, \ldots, \psi_{s}\right)$ so

$$
\begin{array}{r}
C_{g}^{+}\left(\psi_{1}, \ldots, \psi_{s}\right)-\operatorname{div}_{i} \sum_{h \in H} a_{h} C_{g}^{h, i}\left(\psi_{1}, \ldots, \psi_{s}\right) \\
=C_{g}^{\sharp}\left(\psi_{1}, \ldots, \psi_{s}\right)+\sum_{z \in Z^{\prime}} a_{z} C_{g}^{z}\left(\psi_{1}, \ldots, \psi_{s}\right),
\end{array}
$$


modulo complete contractions of length $\geq \sigma+1$ and also:

$$
C_{g}^{++, h}\left(\psi_{1}, \ldots, \psi_{s}\right)-\operatorname{div}_{i} \sum_{h \in H} a_{h} C_{g}^{h, i}\left(\psi_{1}, \ldots, \psi_{s}\right)=\sum_{z \in Z^{\prime}} a_{z} C_{g}^{z}\left(\psi_{1}, \ldots, \psi_{s}\right),
$$

modulo complete contractions of length $\geq \sigma+1$.

We will now derive (3.15), (3.16), (3.17), (3.18), (3.19), (3.20), (3.21). Before outlining the proof, however, we note that once we show the above equations, we can derive that there is a linear combination of acceptable vector fields, $\sum_{h \in H} a_{h} C_{g}^{h, i}\left(\psi_{1}, \ldots, \psi_{s}\right)$, so that:

$$
\begin{aligned}
& \sum_{f \in F_{|\Delta|=\sigma-1}^{\sigma} \cup F_{|\Delta|=\sigma-1}^{\sigma-1} \cup F_{|\Delta|=\sigma-1}^{\sigma-2} \cup F_{|\Delta|=\sigma-2}^{\sigma} \cup F_{|\Delta|=\sigma-2}^{\sigma-1} \cup F_{|\Delta|=\sigma-2}^{\sigma-2}} a_{f} C_{g}^{f}\left(\psi_{1}, \ldots, \psi_{s}\right) \\
& -\operatorname{div}_{i} \sum_{h \in H} a_{h} C_{g}^{h, i}\left(\psi_{1}, \ldots, \psi_{s}\right)=(\text { const })_{*} \cdot C_{g}^{*}\left(\psi_{1}, \ldots, \psi_{s}\right) \\
& +\sum_{y=1}^{s}(\text { const })_{y, *} \cdot C_{g}^{y, *}\left(\psi_{1}, \ldots, \psi_{s}\right)+\sum_{w=1}^{s} \sum_{q=1}^{w-1}(\text { const })_{w, q} \cdot C_{g}^{w, q}\left(\psi_{1}, \ldots, \psi_{s}\right) \\
& +(\text { const })_{\sharp} C_{g}^{\sharp}\left(\psi_{1}, \ldots, \psi_{s}\right)+\sum_{z \in Z^{\prime}} a_{z} C_{g}^{z}\left(\psi_{1}, \ldots, \psi_{s}\right) .
\end{aligned}
$$

Proof of (3.15), (3.16), (3.17), (3.18), (3.19), (3.20), (3.21). The divergences needed in all the above equations are constructed "by hand", by consecutively picking derivative indices, making them into free indices and taking the corresponding divergence. As the proof is essentially the same for the equations (3.15), (3.16), (3.17), (3.18), (3.19), we will only demonstrate the first one. Afterwards, we show (3.20) and (3.21).

We consider a complete contraction $C_{g}^{f}\left(\psi_{1}, \ldots, \psi_{s}\right)$ in the form (3.10). We first show that modulo introducing complete contractions of length $\geq \sigma+1$, we can subtract a divergence $\operatorname{div}_{i} \sum_{h \in H} a_{h} C_{g}^{h, i}\left(\psi_{1}, \ldots, \psi_{s}\right)$ from $C_{g}^{f}\left(\psi_{1}, \ldots, \psi_{s}\right)$ and obtain:

$$
\begin{aligned}
& C_{g}^{f}\left(\psi_{1}, \ldots, \psi_{s}\right)-\operatorname{div}_{i} \sum_{h \in H} a_{h} C_{g}^{h, i}\left(\psi_{1}, \ldots, \psi_{s}\right)=\sum_{v=2}^{\frac{n}{2}-\sigma-4}(\text { const })_{f, v} \cdot C_{g}^{v, \tau}\left(\psi_{1}, \ldots, \psi_{s}\right) \\
& \quad+\sum_{f \in F_{|\Delta|=\sigma-2, \beta}^{\sigma-s}} a_{f} C_{g}^{f}\left(\psi_{1}, \ldots, \psi_{s}\right)+\sum_{z \in Z^{\prime}} a_{z} C_{g}^{z}\left(\psi_{1}, \ldots, \psi_{s}\right)
\end{aligned}
$$

where $C_{g}^{v, \tau}\left(\psi_{1}, \ldots, \psi_{s}\right)$ stands for a complete contraction in the form (3.10) where and all the indices $r_{1}, \ldots, r_{p}, i, j$ and $z_{1}, \ldots, z_{q}, i^{\prime}, j^{\prime}$ are contracting against a derivative index (such a complete contraction has $\delta \geq \mu+2$ ) and also the first factor has $t=v$.

We observe that once we show the above, we can then repeat the proof of equation (3.9) to this setting to find a divergence $\operatorname{div}_{i} \sum_{h \in H} a_{h} C_{g}^{h, i}\left(\psi_{1}, \ldots, \psi_{s}\right)$ so that for each $v$ :

$$
\begin{array}{r}
C_{g}^{v, \tau}\left(\psi_{1}, \ldots, \psi_{s}\right)-\operatorname{div}_{i} \sum_{h \in H} a_{h} C_{g}^{h, i}\left(\psi_{1}, \ldots, \psi_{s}\right) \\
=C_{g}^{*}\left(\psi_{1}, \ldots, \psi_{s}\right)+\sum_{z \in Z^{\prime}} a_{z} C_{g}^{z}\left(\psi_{1}, \ldots, \psi_{s}\right),
\end{array}
$$

modulo complete contractions of length $\geq \sigma+1$.

Then, combining equations (3.23) and (3.24), we will deduce (3.15).

We show (3.23) by an induction. Firstly let us observe that the maximum value that $\delta$ can have for a complete contraction in the form (3.10) is $\frac{n}{2}+(\sigma-s)$. If $\delta=\frac{n}{2}+(\sigma-s)$ then all indices appearing in (3.10) must be involved in an internal contraction (and thus there is nothing 
to show). Now, let us suppose that $C_{g}^{f}\left(\psi_{1}, \ldots, \psi_{s}\right)$ has $\delta=P \geq \mu+2,{ }^{25} P<\frac{n}{2}+(\sigma-s)$. We then construct a vector field $C_{g}^{h, i}\left(\psi_{1}, \ldots, \psi_{s}\right)$ so that modulo complete contractions of length $\geq \sigma+1$ :

$$
\begin{aligned}
& C_{g}^{f}\left(\psi_{1}, \ldots, \psi_{s}\right)-\operatorname{div}_{i} C_{g}^{h, i}\left(\psi_{1}, \ldots, \psi_{s}\right) \\
& \quad=\left(C^{f}\right)_{g}^{\prime}\left(\psi_{1}, \ldots, \psi_{s}\right)+\sum_{f \in F_{|\Delta|=\sigma-2, \beta}^{\sigma-s}} a_{f} C_{g}^{f}\left(\psi_{1}, \ldots, \psi_{s}\right)+\sum_{z \in Z^{\prime}} a_{z} C_{g}^{z}\left(\psi_{1}, \ldots, \psi_{s}\right) .
\end{aligned}
$$

Here $\left(C^{f}\right)_{g}^{\prime}\left(\psi_{1}, \ldots, \psi_{s}\right)$ is a complete contraction in the form (3.10) with $\delta=P+1$. Clearly, if we can show the above then by iterative repetition (3.23) will follow.

The vector field $C_{g}^{h, i}\left(\psi_{1}, \ldots, \psi_{s}\right)$ needed for (3.25) can be easily constructed. We observe that since $\sigma<\frac{n}{2}-1$ and the weight is $-n$, we have that at least one of the first two factors in (3.10) has at least four indices. With no loss of generality, we assume it is the first factor, $\nabla^{a_{1} \ldots a_{t}} \nabla_{r_{1} \ldots r_{p}}^{(p)} \operatorname{Ric}_{i j}$ for which $t+p+2 \geq 4$. Moreover, since we are assuming $\delta<\frac{n}{2}+(\sigma-s)$, it follows that at least one of the indices $r_{1}, \ldots, r_{p}, i, j$ is not contracting against an index $a_{1}, \ldots, a_{t}$. That index can either be a derivative index ( say $_{r_{1}}$ with no loss of generality) or-if all the indices $r_{1}, \ldots, r_{p}$ are each contracting against one of the indices $a_{1}, \ldots, a_{t}-$ an internal index (say $i$ with no loss of generality).

In the first case, we define $C_{g}^{h, i}\left(\psi_{1}, \ldots, \psi_{s}\right)$ to be the vector field obtained from $C^{f}$ be erasing the derivative index $r_{1}$ in the first factor and making the index ${ }^{r_{1}}$ in the second factor in (3.10) into a free index. We check that for this vector field, (3.25) indeed holds.

In the second case, we see that since $t+p+2 \geq 4$, we can apply the second Bianchi identity (modulo introducing a complete contraction that will belong to the linear combination $\left.\sum_{f \in F_{|\Delta|=\sigma-2, \beta}^{\sigma-s}} a_{f} C_{g}^{f}\left(\psi_{1}, \ldots, \psi_{s}\right)\right)$ and be reduced to the previous case, where the index $r_{1}$ is not contracting against one of the indices $a_{1}, \ldots, a_{t}$. Thus, we have proven (3.15). The equations (3.16), (3.17), (3.18), (3.19) follow by essentially the same reasoning.

We now show $(3.20),(3.21)$. In both cases, we define $C_{g}^{b, l}\left(\psi_{1}, \ldots, \psi_{s}\right)$ to stand for the vector field obtained from $C_{g}^{+}\left(\psi_{1}, \ldots, \psi_{s}\right)$ and $C_{g}^{++, h}\left(\psi_{1}, \ldots, \psi_{s}\right)$ respectively, by erasing the derivative index ${ }^{i}$ in the first factor. Then, for (3.21) we define

$$
\sum_{h \in H} a_{h} C_{g}^{h, i}\left(\psi_{1}, \ldots, \psi_{s}\right)=C_{g}^{b, i}\left(\psi_{1}, \ldots, \psi_{s}\right)
$$

Since $C^{++, h}$ has $\delta \geq \mu+2$, we observe that if $\nabla_{i}$ in $\operatorname{div}_{i} C_{g}^{b, i}\left(\psi_{1}, \ldots, \psi_{s}\right)$ hits the first factor, we cancel out the complete contraction $C^{++, h}$. When $\nabla_{i}$ hits the second factor, we get a complete contraction that is equal to a complete contraction of length $\sigma+1$ (this is due to the antisymmetry of the indices $i, j$ ). If it hits one of the other factors, we get a complete contraction that belongs to the linear combination $\sum_{z \in Z^{\prime}} a_{z} C_{g}^{z}\left(\psi_{1}, \ldots, \psi_{s}\right)$.

The case of (3.20) is more complicated. We again consider the same vector field $C_{g}^{b, i}\left(\psi_{1}\right.$, $\left.\ldots, \psi_{s}\right)$ as above. We then observe that modulo complete contractions of length $\geq \sigma+1$ :

$$
C_{g}^{+}\left(\psi_{1}, \ldots, \psi_{s}\right)-\operatorname{div}_{i} C_{g}^{b, i}\left(\psi_{1}, \ldots, \psi_{s}\right)=C_{g}^{\prime}\left(\psi_{1}, \ldots, \psi_{s}\right)+\sum_{z \in Z^{\prime}} a_{z} C_{g}^{z}\left(\psi_{1}, \ldots, \psi_{s}\right),
$$

where $C^{\prime}$ is the complete contraction:

$$
\operatorname{contr}\left(\Delta^{\frac{n}{2}-1-\sigma} \nabla^{l} R_{i j k l} \otimes \nabla^{l^{\prime}} R^{i j k} l^{\prime} \otimes R^{\sigma-s-2} \otimes \Delta \psi_{1} \otimes \cdots \otimes \Delta \psi_{s}\right) .
$$

\footnotetext{
${ }^{25}$ This inequality holds by virtue of the "Important remark" in the introduction, and because of the fact that $s<\sigma-2$.
} 
Notice that since $\sigma<\frac{n}{2}-1$, we have $t>1$ derivatives on the first factor. We then apply the second Bianchi identity and write:

$$
\begin{aligned}
C_{g}^{\prime}\left(\psi_{1}, \ldots, \psi_{s}\right)= & \operatorname{contr}\left(\Delta^{\frac{n}{2}-1-\sigma-1} \nabla^{i t l} R_{t j k l} \otimes \nabla^{l^{\prime}} R_{i}{ }^{j k}{ }^{\prime}\right. \\
& \left.\otimes R^{\sigma-s-2} \otimes \Delta \psi_{1} \otimes \cdots \otimes \Delta \psi_{s}\right) .
\end{aligned}
$$

We then define $C_{g}^{c, i}\left(\psi_{1}, \ldots, \psi_{s}\right)$ to stand for the vector field that arises from the right hand side of the above by erasing the index ${ }^{i}$ in the first factor and making the index ${ }_{i}$ that it contracted against in the second factor into a free index. We observe that:

$$
C_{g}^{\prime}\left(\psi_{1}, \ldots, \psi_{s}\right)-\operatorname{div}_{i} C_{g}^{c, i}\left(\psi_{1}, \ldots, \psi_{s}\right)=C_{g}^{\sharp}\left(\psi_{1}, \ldots, \psi_{s}\right)+\sum_{z \in Z^{\prime}} a_{z} C_{g}^{z}\left(\psi_{1}, \ldots, \psi_{s}\right) .
$$

Therefore, combining (3.26), (3.27) and (3.28) we have that the vector field needed for (3.20) is precisely:

$$
\sum_{h \in H} a_{h} C_{g}^{h, i}\left(\psi_{1}, \ldots, \psi_{s}\right)=C_{g}^{b, i}\left(\psi_{1}, \ldots, \psi_{s}\right)-C_{g}^{c, i}\left(\psi_{1}, \ldots, \psi_{s}\right) .
$$

The third step of the proof of Lemma 3.1. In view of equation (3.22), by subtracting the divergence $\operatorname{div}_{i} \sum_{h \in H} a_{h} C_{g}^{h, i}\left(\psi_{1}, \ldots, \psi_{s}\right)$ from $I_{g}^{s}\left(\psi_{1}, \ldots, \psi_{s}\right)$, we have obtained a relation, modulo complete contractions of length $\geq \sigma+1$ :

$$
\begin{aligned}
& I_{g}^{s}\left(\psi_{1}, \ldots, \psi_{s}\right)-\operatorname{div}_{i} \sum_{h \in H} a_{h} C_{g}^{h, i}\left(\psi_{1}, \ldots, \psi_{s}\right)=\sum_{l \in L_{\mu}} a_{l} C_{g}^{l, \iota}\left(\psi_{1}, \ldots, \psi_{s}\right) \\
& \quad+\sum_{j \in J} a_{j} C_{g}^{j}\left(\psi_{1}, \ldots, \psi_{s}\right)+\sum_{q_{1}=1}^{\sigma-s-3} \sum_{f \in F^{q_{1}}} a_{f} C_{g}^{f}\left(\psi_{1}, \ldots, \psi_{s}\right)+(\text { const })_{*} \cdot C_{g}^{*}\left(\psi_{1}, \ldots, \psi_{s}\right) \\
& \quad+\sum_{y=1}^{s}(\mathrm{const})_{y, *} \cdot C_{g}^{y, *}\left(\psi_{1}, \ldots, \psi_{s}\right)+\sum_{w=1}^{s} \sum_{q=1}^{w-1}(\text { const })_{w, q} \cdot C_{g}^{w, q}\left(\psi_{1}, \ldots, \psi_{s}\right) \\
& \quad+(\text { const })_{\sharp} C_{g}^{\sharp}\left(\psi_{1}, \ldots, \psi_{s}\right)+\sum_{z \in Z^{\prime}} a_{z} C_{g}^{z}\left(\psi_{1}, \ldots, \psi_{s}\right) .
\end{aligned}
$$

We next claim that $(\text { const })_{*}$, (const $)_{y, *},(\text { const })_{w, q},(\text { const })_{\sharp}=0$; if we can prove this, we will then have proven Lemma 3.1 in this case where $s<\sigma-2$.

We first prove that $(\text { const })_{*}=0$. We denote the r.h.s. of the above by $Z_{g}\left(\psi_{1}, \ldots, \psi_{s}\right)$. Clearly, $\int_{M^{n}} Z_{g}\left(\psi_{1}, \ldots, \psi_{s}\right) d V_{g}=0$.

We now apply the "main conclusion" of the super divergence formula to this integral equation (see [1]), deriving an equation $\operatorname{supdiv}\left[I_{g}^{s}\left(\psi_{1}, \ldots, \psi_{s}, \Omega^{\sigma-s}\right)\right]=0 .{ }^{26}$ We focus on the sublinear combination $\operatorname{supdiv}_{*}\left[I_{g}^{s}\left(\psi_{1}, \ldots, \psi_{s}, \Omega^{\sigma-s}\right)\right]$ of complete contractions with $\delta=0, s$ factors $\nabla \psi_{h}$ and $\sigma-s-2$ factors $\nabla \Omega$. Since the "main conclusion" of the super divergence formula holds formally, it follows that:

$$
\operatorname{supdiv}_{*}\left[Z_{g}\left(\psi_{1}, \ldots, \psi_{s}, \Omega^{\sigma-s}\right)\right]=0,
$$

modulo complete contractions of length $\geq \sigma+1$.

Since each $C^{f}$ in (3.29) has $|\Delta| \leq \sigma-3$, it follows that:

$$
\operatorname{supdiv}_{*}\left[Z_{g}\left(\psi_{1}, \ldots, \psi_{s}, \Omega^{\sigma-s}\right)\right]=(-1)^{\frac{n}{2}}(\text { const })_{*} \cdot C_{g}^{x}\left(\psi_{1}, \ldots, \psi_{s}\right),
$$

\footnotetext{
${ }^{26}$ Recall that $I_{g}^{s}\left(\psi_{1}, \ldots, \psi_{s}, \Omega^{\sigma-s}\right)$ stands for the linear combination that arises from $I_{g}^{s}\left(\psi_{1}, \ldots, \psi_{s}\right)$ by formally replacing each factor $\nabla_{r_{1} \ldots r_{p}}^{(p)} \operatorname{Ric}_{i j} \neq R$ by $-\nabla_{r_{1} \ldots r_{p} i j}^{(p+2)} \Omega$ and each factor $R$ by $-2 \Delta \Omega$.
} 
where $C_{g}^{x}\left(\psi_{1}, \ldots, \psi_{s}\right)$ is the expression:

$$
\begin{array}{r}
\operatorname{contr}\left(\nabla^{y_{1} \ldots y_{\sigma-s-2} w_{1} \ldots w_{s}}\left(\nabla^{x_{1} \ldots x \frac{n}{2}-\sigma+2} \Omega \otimes \nabla_{x_{1} \ldots x_{\frac{n}{2}-\sigma+2}} \Omega\right)\right. \\
\left.\otimes \nabla_{y_{1}} \Omega \otimes \cdots \otimes \nabla_{y_{\sigma-s-2}} \Omega \otimes \nabla_{w_{1}} \psi_{1} \otimes \cdots \otimes \nabla_{w_{s}} \psi_{s}\right) .
\end{array}
$$

Since this complete contraction is clearly not zero, we deduce that (const) ${ }_{*}=0$.

Showing that the other constants in (3.29) are zero follows essentially the same pattern. We next show that each (const) $)_{y, *}=0$. In order to do that, we again consider the main conclusion of the super divergence formula applied to $Z_{g}\left(\psi_{1}, \ldots, \psi_{s}\right), \operatorname{supdiv}\left[Z_{g}\left(\psi_{1}, \ldots, \psi_{s}, \Omega^{\sigma-s}\right)\right]=0$. We pick out the sublinear combination $\operatorname{supdiv}_{* *, y}\left[Z_{g}\left(\psi_{1}, \ldots, \psi_{s}, \Omega^{\sigma-s}\right)\right]$ of complete contractions with length $\sigma, \delta=0$ and $s-1$ factors $\nabla \psi_{h}, h=1, \ldots, \hat{y}, \ldots, s$ and $\sigma-2-(s-1)$ factors $\nabla \Omega$. Since the main conclusion of the super divergence holds formally, we deduce that:

$$
\operatorname{supdiv}_{* *, y}\left[I_{g}^{s}\left(\psi_{1}, \ldots, \psi_{s}, \Omega^{\sigma-s}\right)\right]=0,
$$

modulo complete contractions of length $\geq \sigma+1$.

Analogously to the previous case, we deduce that:

$$
\operatorname{supdiv}_{* *, y}\left[I_{g}^{s}\left(\psi_{1}, \ldots, \psi_{s}, \Omega^{\sigma-s}\right)\right]=(-1)^{\frac{n}{2}}(\text { const })_{y, *} \cdot C_{g}^{x x}\left(\psi_{1}, \ldots, \psi_{s}\right),
$$

where $C_{g}^{x x}\left(\psi_{1}, \ldots, \psi_{s}\right)$ is the complete contraction:

$$
\begin{aligned}
& \operatorname{contr}\left(\nabla^{y_{1} \ldots y_{\sigma-s-1} w_{1} \ldots \hat{w}_{y} \ldots w_{s}}\left(\nabla^{x_{1} \ldots x_{\frac{n}{2}-\sigma+2}} \Omega \otimes \nabla_{x_{1} \ldots x \frac{n}{2}-\sigma+2} \psi_{y}\right)\right. \\
& \left.\quad \otimes \nabla_{y_{1}} \Omega \otimes \cdots \otimes \nabla_{y_{\sigma-s-1}} \Omega \otimes \nabla_{w_{1}} \psi_{1} \otimes \cdots \otimes \nabla \hat{\psi}_{y} \otimes \cdots \otimes \nabla_{w_{s}} \psi_{s}\right) .
\end{aligned}
$$

Therefore, we deduce that each (const) $)_{y, *}=0$.

To prove that (const) $)_{w, q}=0$, we again consider $Z_{g}\left(\psi_{1}, \ldots, \psi_{s}, \Omega^{\sigma-s}\right),{ }^{27}$ for which $\int_{M^{n}} Z_{g}\left(\psi_{1}, \ldots, \psi_{s}, \Omega^{\sigma-s}\right)=0$. We apply the super divergence formula to this equation (see [1]), deriving a new local equation, $\sup \operatorname{div}\left[Z_{g}\left(\psi_{1}, \ldots, \psi_{s}, \Omega^{\sigma-s}\right)\right]=0$; we pick out the sublinear combination $\operatorname{supdiv}_{* * *,(w, q)}\left[I_{g}^{s}\left(\psi_{1}, \ldots, \psi_{s}, \Omega^{\sigma-s}\right)\right]$ of complete contractions with length $\sigma, \delta=0$ and $s-2$ factors $\nabla \psi_{h}, h=1, \ldots, \hat{w}, \ldots, \hat{q}, s$ and $\sigma-s$ factors $\nabla \Omega$. Since the super divergence formula holds formally, we deduce that:

$$
\operatorname{supdiv}_{* *,(w, q)}\left[I_{g}^{s}\left(\psi_{1}, \ldots, \psi_{s}, \Omega^{\sigma-s}\right)\right]=0,
$$

modulo complete contractions of length $\geq \sigma+1$.

We now claim that:

$$
\operatorname{supdiv}_{* * *,(w, q)}\left[I_{g}^{s}\left(\psi_{1}, \ldots, \psi_{s}, \Omega^{\sigma-s}\right)\right]=(-1)^{\frac{n}{2}}(\text { const })_{\sharp} \cdot C_{g}^{x x x}\left(\psi_{1}, \ldots, \psi_{s}\right),
$$

where $C_{g}^{x x x}\left(\psi_{1}, \ldots, \psi_{s}\right)$ is the complete contraction:

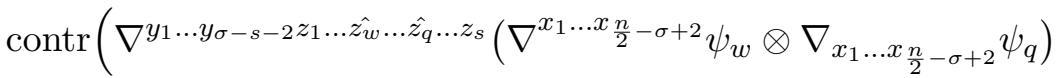

$$
\begin{aligned}
& \left.\otimes \nabla_{y_{1}} \Omega \otimes \cdots \otimes \nabla_{y_{\sigma-s}} \Omega \otimes \nabla_{w_{1}} \psi_{1} \otimes \cdots \otimes \nabla \hat{\psi}_{w} \otimes \cdots \otimes \nabla \hat{\psi_{q}} \otimes \cdots \otimes \nabla_{w_{s}} \psi_{s}\right) .
\end{aligned}
$$

In order to see this, we recall that we have already shown that (const) $)_{*},(\text { const })_{I I, y, *},(\text { const })_{w, q}$ $=0$, and also each other complete contraction $C^{f}$ in (3.29) has $|\Delta| \leq \sigma-3$, hence each complete contraction of length $\sigma$ in each Tail $\left[C^{f}\right]$ can have at most $\sigma-3$ factors $\nabla \Omega$ or $\nabla \psi_{h}$. Therefore, we deduce that $(\text { const })_{(w, q)}=0$.

\footnotetext{
${ }^{27}$ See the "Main consequence" of the super divergence formula in Subsection 2.2.3 in [3].
} 
Finally, to show $(\text { const })_{\sharp}=0$, we again consider $Z_{g}\left(\psi_{1}, \ldots, \psi_{s}, \Omega^{\sigma-s-2}\right),{ }^{28}$ for which we have $\int_{M^{n}} Z_{g}\left(\psi_{1}, \ldots, \psi_{s}, \Omega^{\sigma-s-2}\right) d V_{g}=0$. We apply the super divergence formula to this equation, deriving a new local equation: $\sup \operatorname{div}\left[I_{g}^{s}\left(\psi_{1}, \ldots, \psi_{s}, \Omega^{\sigma-s-2}\right)\right]=0$; we pick out the sublinear combination supdiv $\operatorname{si**}_{* *}\left[Z_{g}\left(\psi_{1}, \ldots, \psi_{s}, \Omega^{\sigma-s-2}\right)\right]$ of complete contractions with length $\sigma, \delta=0$ and $s$ factors $\nabla \psi_{h}$, and $\sigma-s-2$ factors $\nabla \Omega$. Since the super divergence formula holds formally, we deduce that:

$$
\operatorname{supdiv}_{* * * *}\left[Z_{g}\left(\psi_{1}, \ldots, \psi_{s}, \Omega^{\sigma-s-2}\right)\right]=0,
$$

modulo complete contractions of length $\geq \sigma+1$.

Analogously to the previous case, we deduce that:

$$
\operatorname{supdiv}_{* * * *}\left[I_{g}^{s}\left(\psi_{1}, \ldots, \psi_{s}, \Omega^{\sigma-s-2}\right)\right]=(-1)^{\frac{n}{2}}(\text { const })_{\sharp} \cdot C_{g}^{x x x x}\left(\psi_{1}, \ldots, \psi_{s}\right),
$$

where $C_{g}^{x x x x}\left(\psi_{1}, \ldots, \psi_{s}\right)$ is the complete contraction:

$$
\begin{aligned}
& \operatorname{contr}\left(\nabla^{y_{1} \ldots y_{\sigma-s-2} z_{1} \ldots z_{s}}\left(\nabla^{x_{1} \ldots x_{\frac{n}{2}-\sigma-2} i^{\prime} l^{\prime}} R_{j k}^{i}{ }^{l} \otimes \nabla_{x_{1} \ldots x_{\frac{n}{2}-\sigma-2} i l} R_{i^{\prime}}{ }^{j k}{ }_{l^{\prime}}\right)\right. \\
& \left.\otimes \nabla_{y_{1}} \Omega \otimes \cdots \otimes \nabla_{y_{\sigma-s-2}} \Omega \otimes \nabla_{w_{1}} \psi_{1} \otimes \cdots \otimes \nabla_{w_{s}} \psi_{s}\right) .
\end{aligned}
$$

Hence (const) $)_{\sharp}=0$. We have shown Lemma 3.1 in the case $\sigma<\frac{n}{2}-1$.

Proof of Lemma 3.1 in the case $\sigma=\frac{n}{2}-1$. The case $\sigma=\frac{n}{2}-1$ follows similarly. In this case, we can apply the same method of explicitly constructing divergences to show that there is a linear combination of acceptable vector fields, $\sum_{h \in H} a_{h} C_{g}^{h, i}\left(\psi_{1}, \ldots, \psi_{s}\right)$, so that (3.22) holds, where in this setting $C_{g}^{*}\left(\psi_{1}, \ldots, \psi_{s}\right)$ is in the form:

$$
\operatorname{contr}\left(\nabla^{a} \operatorname{Ric}_{a i} \otimes \nabla^{b} \operatorname{Ric}_{b}^{i} \otimes R^{\sigma-s-2} \otimes \Delta \psi_{1} \otimes \cdots \otimes \Delta \psi_{s}\right),
$$

whereas $C_{g}^{y, *}\left(\psi_{1}, \ldots, \psi_{s}\right)$ is in the form:

$$
\operatorname{contr}\left(\nabla^{a} \operatorname{Ric}_{a i} \otimes \nabla_{b}^{b i} \psi_{y} \otimes R^{\sigma-s-2} \otimes \Delta \psi_{1} \otimes \cdots \otimes \Delta \hat{\psi_{y}} \otimes \cdots \otimes \Delta \psi_{s}\right),
$$

and $C_{g}^{q, w}\left(\psi_{1}, \ldots, \psi_{s}\right)$ is in the form:

$$
\operatorname{contr}\left(\nabla_{a}^{a i} \psi_{q} \otimes \nabla_{b}^{b i} \psi_{w} \otimes R^{\sigma-s-2} \otimes \Delta \psi_{1} \otimes \cdots \otimes \Delta \hat{\psi}_{q} \otimes \cdots \otimes \Delta \hat{\psi}_{w} \otimes \cdots \otimes \Delta \psi_{s}\right),
$$

and finally $C_{g}^{\sharp}\left(\psi_{1}, \ldots, \psi_{s}\right)$ is in the form:

$$
\operatorname{contr}\left(\nabla^{a} R_{i j k a} \otimes \nabla^{b} R_{b}^{i j k} \otimes R^{\sigma-s-2} \otimes \Delta \psi_{1} \otimes \cdots \otimes \Delta \psi_{s}\right) .
$$

Therefore, by subtracting the divergence $\operatorname{div}_{i} \sum_{h \in H} a_{h} C_{g}^{h, i}\left(\psi_{1}, \ldots, \psi_{s}\right)$ from $I_{g}^{s}\left(\psi_{1}, \ldots, \psi_{s}\right)$, we again obtain (3.29) (modulo complete contractions of length $\geq \sigma+1$ ), with the new notational conventions of this setting.

Hence, if we could show that $(\text { const })_{*},(\text { const })_{y, *},(\text { const })_{w, q}$, (const $)_{\sharp}=0$, we will then have proven Lemma 3.1 in the case $\sigma=\frac{n}{2}-1$.

Now, we show that $(\text { const })_{*},(\text { const })_{I I, y},(\text { const })_{w, q}=0$. As before, we take $\operatorname{supdiv}\left[Z_{g}\left(\psi_{1}, \ldots\right.\right.$, $\left.\psi_{s}, \Omega^{\sigma-s-2}\right)$ and focus on the same sublinear combinations $\operatorname{supdiv}_{*}, \operatorname{supdiv}_{* *}, \operatorname{supdiv}_{* * *}$ as in the previous case, and then observe that the same equations (3.30), (3.32), (3.35) also hold in this case. The complete contractions $C^{x}, C^{x x}, C^{x x x}$ are the same as in equations (3.31), (3.34), (3.37), where we set $\sigma=\frac{n}{2}-1$.

Now, in order to show that (const) $\sharp=0$, we apply the same method as for the previous case: We consider and focus on the sublinear combination of complete contractions with length $\sigma$,

\footnotetext{
${ }^{28}$ See the "Main consequence" of the super divergence formula in Subsection 2.2.3 in [3].
} 
$\delta=0$, and $s$ factors $\nabla \psi_{h}, \sigma-2-s$ factors $\nabla \Omega$. We denote that sublinear combination by $\operatorname{supdiv}_{* * * *}\left[I_{g}^{s}\left(\psi_{1}, \ldots, \psi_{s}, \Omega^{\sigma-s-2}\right)\right]$. By the same arguments as before, we again deduce an equation (3.38), where $C^{x x x x}$ here stands for the complete contraction:

$$
\begin{aligned}
& \operatorname{contr}\left(\nabla^{y_{1} \ldots y_{\sigma-s-2} z_{1} \ldots z_{s}}\left(\nabla^{l^{\prime}} R_{i j k}^{l} \otimes \nabla_{l} R^{i j k} l_{l^{\prime}}\right)\right. \\
& \left.\quad \otimes \nabla_{y_{1}} \Omega \otimes \cdots \otimes \nabla_{y_{\sigma-s-2}} \Omega \otimes \nabla_{w_{1}} \psi_{1} \otimes \cdots \otimes \nabla_{w_{s}} \psi_{s}\right) .
\end{aligned}
$$

We thus deduce that (const $)_{\sharp}=0$ in this case also.

\subsection{The proof of Lemmas 1.2, 1.3, 1.4 and Lemma 1.5 when $s=\sigma-2$}

We observe that if $\sigma<\frac{n}{2}-1$ then $\mu \leq \frac{n}{2}$. We distinguish subcases, based on $\sigma$ and $\mu$. Our three subcases are $\left(\sigma<\frac{n}{2}-1, \mu<\frac{n}{2}\right),\left(\sigma<\frac{n}{2}-1, \mu=\frac{n}{2}\right)$, and $\sigma=\frac{n}{2}-1$.

Firstly, we consider the subcase where $\sigma<\frac{n}{2}-1$ and $\mu<\frac{n}{2}$.

Proof of Lemma 1.5 when $s=\boldsymbol{\sigma}-\mathbf{2}, \boldsymbol{\sigma}<\frac{\boldsymbol{n}}{\mathbf{2}} \mathbf{-} \mathbf{1}$. Consider $\left.P(g)\right|_{\Theta_{\sigma-2}} \cdot{ }^{29}$ We pick out the sublinear combination of complete contractions in the form:

$$
\operatorname{contr}\left(\nabla^{a_{1} \ldots a_{t}} \nabla^{\left(m_{1}\right)} W_{i j k l} \otimes \nabla^{b_{1} \ldots b_{s}} \nabla^{\left(m_{2}\right)} W_{i^{\prime} j^{\prime} k^{\prime} l^{\prime}} \otimes\left(P_{a}^{a}\right)^{\sigma-2}\right) .
$$

We index those complete contractions in the set $\Theta_{\sigma-2}^{*}$. We then easily see (by repeating the explicit constructions from the previous subsection) that we can subtract a divergence from $\left.P(g)\right|_{\Theta_{\sigma-2}^{*}}$ so that, modulo complete contractions of length $\geq \sigma+1$ :

$$
\left.P(g)\right|_{\Theta_{\sigma-2}^{*}}-\operatorname{div}_{i} \sum_{h \in H} a_{h} C^{h, i}(g)=(\text { const })_{\alpha} C^{\alpha}(g)+\sum_{t \in T} a_{t} C^{t}(g),
$$

where $C^{\alpha}(g)$ in the complete contraction in the form:

$$
\operatorname{contr}\left(\Delta^{\frac{n}{2}-\sigma-2} \nabla^{i l} W_{i j k l} \otimes \nabla^{i^{\prime} l^{\prime}} W_{i^{\prime}}^{j k} l_{l^{\prime}} \otimes\left(P_{a}^{a}\right)^{\sigma-2}\right),
$$

while ${ }^{30} \sum_{t \in T} a_{t} C^{t}(g)$ stands for a linear combination of complete contractions in the form:

$$
\operatorname{contr}\left(\nabla^{a_{1} \ldots a_{t}} \nabla^{\left(m_{1}\right)} W_{i j k l} \otimes \nabla^{b_{1} \ldots b_{s}} \nabla^{\left(m_{2}\right)} W_{i^{\prime} j^{\prime} k^{\prime} l^{\prime}} \otimes \nabla^{f} P_{f i} \otimes\left(P_{a}^{a}\right)^{\sigma-3}\right)
$$

(with fewer than $\sigma-2$ factors $P_{a}^{a}$ and with $\delta \geq \mu$ ).

Therefore, from now on we may assume with no loss of generality that the sublinear combination $\left.P(g)\right|_{\Theta_{\sigma-2}^{*}}$ is precisely (const) ${ }_{\alpha} C^{\alpha}(g)$.

Proof of Lemmas 1.2, 1.3, 1.4 when $s=\sigma-2, \sigma<\frac{n}{2}-1, \mu<\frac{n}{2}$. We again observe that the complete contractions in $I_{g}^{s}\left(\psi_{1}, \ldots, \psi_{s}\right)$ with $|\Delta| \geq \sigma-2$ will be indexed in the sets $F^{2}, F^{1}$ and ${ }^{31}$ in this case, also in the sets $J$ and $L$, where for each $C^{l}, l \in L$ and each $C^{j}$, $j \in J$ we recall that $C^{l}, C^{j}$ are in the form (1.4) with no factors $\nabla^{(p)}$ Ric.

We now again consider $I_{g}^{s}\left(\psi_{1}, \ldots, \psi_{s}\right)$, written out as a linear combination in the form (1.5).

We recall the definition of the index set $F^{*}$ (see the notation above Lemma 1.2). It follows that $F^{*}=F_{\sigma-1}^{2} \bigcup F_{\sigma-2}^{2} \bigcup F_{\sigma-2}^{1}$ (recall that the upper labels count the number of factors $\nabla^{(p)}$ Ric or $R$ and the lower labels count the value of $|\Delta|)$. Furthermore, since $\left.P(g)\right|_{\Theta_{s}}$ is "good", it follows that $L_{\mu}^{*}=\varnothing$, while $\sum_{j \in J^{*}} a_{j} C_{g}^{j}\left(\psi_{1}, \ldots, \psi_{s}\right)=($ const $) C_{g}\left(\psi_{1}, \ldots, \psi_{s}\right)$, where $C_{g}\left(\psi_{1}, \ldots, \psi_{s}\right)$ is the

\footnotetext{
${ }^{29}$ Recall that $\Theta_{\sigma-2}$ stands for the index set of complete contractions in $P(g)$ (which are in the form $\operatorname{contr}\left(\nabla^{(m)} W \otimes \cdots \otimes \nabla^{\left(m^{\prime}\right)} W \otimes \nabla^{(a)} P \otimes \cdots \otimes \nabla^{\left(a^{\prime}\right)} P\right)$ with length $\sigma$ and with $\sigma-2$ factors $\nabla^{(u)} P$.

${ }^{30}$ If $\sigma=\frac{n}{2}-1$ then $C^{\alpha}(g)$ is in the form $\operatorname{contr}\left(\nabla^{l} W_{i j k l} \otimes \nabla_{l^{\prime}} W^{i j k l^{\prime}} \otimes\left(P_{a}^{a}\right)^{\sigma-2}\right)$.

${ }^{31}$ Recall that $F^{q} \subset F$ stands for the index set of complete contractions with $q$ factors $\nabla^{(p)}$ Ric or $R$.
} 
complete contraction $\operatorname{contr}\left(\Delta^{\frac{n}{2}-\sigma-2} \nabla^{i l} R_{i j k l} \otimes \nabla_{i^{\prime} l^{\prime}} R^{i^{\prime} j k l^{\prime}} \otimes \Delta \psi_{1} \otimes \cdots \otimes \Delta \psi_{s}\right)$ or $\operatorname{contr}\left(\nabla^{i} R_{i j k l} \otimes\right.$ $\left.\nabla_{i^{\prime}} R^{i^{\prime} j k l} \otimes \Delta \psi_{1} \otimes \cdots \otimes \Delta \psi_{s}\right)$.

A study of the sublinear combination $\sum_{f \in F_{\sigma-1}^{2}} a_{f} C_{g}^{f}\left(\psi_{1}, \ldots, \psi_{s}\right)$. As before, we see that since $|\Delta|=\sigma-1, s=\sigma-2$ for each $C^{f}, f \in F_{\sigma-1}^{2},{ }^{32}$ it follows that each $C^{f}, f \in F_{\sigma-1}^{2}$ must have at least one factor $R$ and will hence have $\delta\left(C^{f}\right) \geq \mu+2$ (by the decomposition of the Weyl tensor).

As before, it follows that:

$$
\sum_{f \in F_{\sigma-1}^{2}} a_{f} C_{g}^{f}\left(\psi_{1}, \ldots, \psi_{s}\right)=(\text { const })_{@} C_{g}^{@}\left(\psi_{1}, \ldots, \psi_{s}\right)+\sum_{u=1}^{\sigma-2}(\text { const })_{u} C_{g}^{u}\left(\psi_{1}, \ldots, \psi_{s}\right),
$$

where here $C_{g}^{@}$ is the complete contraction:

$$
\operatorname{contr}\left(\Delta^{\left(\frac{n}{2}-\sigma\right)} R \otimes R \otimes \Delta \psi_{1} \otimes \cdots \otimes \Delta \psi_{\sigma-2}\right),
$$

and $C_{g}^{u}\left(\psi_{1}, \ldots, \psi_{s}\right)$ is the complete contraction:

$$
\operatorname{contr}\left(\Delta^{\left(\frac{n}{2}-\sigma+1\right)} \psi_{u} \otimes R^{2} \otimes \Delta \psi_{1} \otimes \Delta \hat{\psi}_{u} \otimes \cdots \otimes \Delta \psi_{\sigma-2}\right) .
$$

As before, we denote by $\sum_{z \in Z^{\prime}} a_{z} C_{g}^{z}\left(\psi_{1}, \ldots, \psi_{s}\right)$ a generic linear combination of complete contractions in the form (1.4) with length $\sigma,|\Delta| \leq \sigma-3$ and $\delta \geq \mu+1$. It follows as in the previous subsection that we can construct a vector field $\sum_{h \in H} a_{h} C_{g}^{h, i}\left(\psi_{1}, \ldots, \psi_{s}\right)$ so that, modulo complete contractions of length $\geq \sigma+1$ :

$$
\begin{aligned}
& \sum_{f \in F_{\sigma-1}^{2}} a_{f} C_{g}^{f}\left(\psi_{1}, \ldots, \psi_{s}\right)-\operatorname{div}_{i} \sum_{h \in H} a_{h} C_{g}^{h, i}\left(\psi_{1}, \ldots, \psi_{s}\right) \\
& \quad=(\text { const })_{!} C_{g}^{!}\left(\psi_{1}, \ldots, \psi_{s}\right)+\sum_{u=1}^{\sigma-2}(\text { const })_{!, u} C_{g}^{!, u}\left(\psi_{1}, \ldots, \psi_{s}\right) \\
& \quad+\sum_{q=1}^{\sigma-2} \sum_{w=1}^{q-1}(\mathrm{const})_{!,(q, w)} C_{g}^{!,(q, w)}\left(\psi_{1}, \ldots, \psi_{s}\right)+\sum_{z \in Z^{\prime}} a_{z} C_{g}^{z}\left(\psi_{1}, \ldots, \psi_{s}\right),
\end{aligned}
$$

where $C_{g}^{!}\left(\psi_{1}, \ldots, \psi_{s}\right)$ is the complete contraction:

$$
\operatorname{contr}\left(\Delta^{\left(\frac{n}{2}-\sigma-1\right)} R \otimes \Delta R \otimes \Delta \psi_{1} \otimes \cdots \otimes \Delta \psi_{\sigma-2}\right),
$$

$C_{g}^{!, u}\left(\psi_{1}, \ldots, \psi_{s}\right)$ is the complete contraction:

$$
\operatorname{contr}\left(\Delta^{\left(\frac{n}{2}-\sigma-1\right)} R \otimes \Delta^{2} \psi_{u} \otimes R \otimes \Delta \psi_{1} \otimes \Delta \hat{\psi}_{u} \otimes \cdots \otimes \Delta \psi_{\sigma-2}\right),
$$

$C_{g}^{!,(q, w)}\left(\psi_{1}, \ldots, \psi_{s}\right)$ is the complete contraction:

$$
\operatorname{contr}\left(\Delta^{\left(\frac{n}{2}-\sigma\right)} \psi_{q} \otimes R^{2} \otimes \Delta^{2} \psi_{w} \otimes \Delta \psi_{1} \otimes \Delta \hat{\psi}_{w} \otimes \cdots \otimes \Delta \hat{\psi_{q}} \otimes \cdots \otimes \Delta \psi_{\sigma-2}\right) .
$$

This follows by the same explicit constructions as for equation (3.4).

A study of the sublinear combination $\sum_{f \in F_{\sigma-2}^{2}} a_{f} C_{g}^{f}\left(\psi_{1}, \ldots, \psi_{s}\right)$. We claim that $\sum_{f \in F_{\sigma-2}^{2}} a_{f} C_{g}^{f}\left(\psi_{1}, \ldots, \psi_{s}\right)$ can be expressed as:

$$
\sum_{f \in F_{\sigma-2}^{2}} a_{f} C_{g}^{f}\left(\psi_{1}, \ldots, \psi_{s}\right)=\sum_{v \in V_{1}} a_{v} C_{g}^{v}\left(\psi_{1}, \ldots, \psi_{s}\right)+\sum_{v \in V_{2}} a_{v} C_{g}^{v}\left(\psi_{1}, \ldots, \psi_{s}\right)
$$

\footnotetext{
${ }^{32}$ Recall that the subscript $\sigma-1$ means that $|\Delta|=\sigma-1$ for the complete contractions indexed in $F_{\sigma-1}^{2}$.
} 


$$
+\sum_{v \in V_{3}} a_{v} C_{g}^{v}\left(\psi_{1}, \ldots, \psi_{s}\right)+(\text { const })_{\mathrm{spec}} \cdot C_{g}^{\mathrm{spec}}\left(\psi_{1}, \ldots, \psi_{s}\right),
$$

where $\sum_{v \in V_{1}} a_{v} C_{g}^{v}\left(\psi_{1}, \ldots, \psi_{s}\right)$ stands for a linear combination of complete contractions in the form:

$$
\begin{aligned}
& \operatorname{contr}\left(\nabla^{a_{1} \ldots a_{t}} \nabla_{r_{1} \ldots r_{p}}^{(p)} \psi_{q} \otimes \nabla^{b_{1} \ldots b_{y}} \nabla_{z_{1} \ldots z_{q}}^{(q)} \psi_{w} \otimes R^{2}\right. \\
& \left.\otimes \Delta \psi_{1} \otimes \cdots \otimes \Delta \hat{\psi}_{q} \otimes \cdots \otimes \Delta \hat{\psi}_{w} \otimes \cdots \otimes \Delta \psi_{s}\right),
\end{aligned}
$$

with $\delta \geq \mu+2 . \sum_{v \in V_{2}} a_{v} C_{g}^{v}\left(\psi_{1}, \ldots, \psi_{s}\right)$ is a linear combination of complete contractions in the form:

$$
\operatorname{contr}\left(\nabla^{a_{1} \ldots a_{t}} \nabla_{r_{1} \ldots r_{p}}^{(p)} \psi_{u} \otimes \nabla^{b_{1} \ldots b_{y}} \nabla_{z_{1} \ldots z_{q}}^{(q)} \operatorname{Ric}_{i j} \otimes R \otimes \Delta \psi_{1} \otimes \cdots \otimes \Delta \hat{\psi}_{u} \otimes \cdots \otimes \Delta \psi_{s}\right),
$$

with $\delta \geq \mu+2$. $\sum_{v \in V_{3}} a_{v} C_{g}^{v}\left(\psi_{1}, \ldots, \psi_{s}\right)$ is a linear combination of complete contractions in the form:

$$
\operatorname{contr}\left(\nabla^{a_{1} \ldots a_{t}} \nabla_{r_{1} \ldots r_{p}}^{(p)} \operatorname{Ric}_{i j} \otimes \nabla^{b_{1} \ldots b_{y}} \nabla_{z_{1} \ldots z_{q}}^{(q)} \operatorname{Ric}_{i^{\prime} j^{\prime}} \otimes \Delta \psi_{1} \otimes \cdots \otimes \Delta \psi_{s}\right),
$$

with $\delta \geq \mu+2$. Finally, $C_{g}^{\text {spec }}\left(\psi_{1}, \ldots, \psi_{s}\right)$ stands for a complete contraction in the form:

$$
\operatorname{contr}\left(\Delta^{\left(\frac{n}{2}-\sigma-2\right)} \nabla_{i j} R \otimes \nabla^{i j} R \otimes \Delta \psi_{1} \otimes \cdots \otimes \Delta \psi_{s}\right),
$$

where $\delta \geq \mu+1$.

(3.40) follows by the definition of $\sum_{f \in F} \cdots$, apart for the claims regarding the numbers of internal contractions in the different factors. We check these claims by virtue of our assumptions on $I_{g}^{s}$. Firstly, polarizing the function $\psi$, we write out $I_{g}^{S}$ as a linear combination of contractions in the form:

$$
\begin{gathered}
\operatorname{contr}\left(\nabla^{a_{1} \ldots a_{t}} \nabla^{(m)} W_{i j k l} \otimes \cdots \otimes \nabla^{b_{1} \ldots b_{u}} \nabla^{\left(m_{\sigma-s}\right)} W_{i^{\prime} j^{\prime} k^{\prime} l^{\prime}}\right. \\
\left.\otimes \nabla^{v_{1} \ldots v_{x}} \nabla^{\left(p_{1}+2\right)} \psi \otimes \cdots \otimes \nabla^{y_{1} \ldots y_{w}} \nabla^{\left(p_{s}+2\right)} \psi\right) .
\end{gathered}
$$

Now, using the decomposition of the Weyl tensor we decompose the above complete contractions. As noted in the introduction, we observe that each complete contraction with a factor $R$ must have $\delta \geq \mu+2$, This shows our claim for (3.41) and (3.42). Moreover, we recall that all complete contractions $C^{l}(g), l \in \Theta_{\sigma-2}$ with $s$ factors $P_{a}^{a}$ are in the form $C^{\alpha}$, as in (3.39). Hence, by applying the decomposition of the Weyl tensor to the complete contraction $C^{\alpha}$, we see that the contribution of $C^{\alpha}$ to the linear combination $\sum_{f \in F_{\sigma-2}^{2}} a_{f} C^{f}$ is precisely the complete contraction $C^{\text {spec }}$ (times a constant). For each other complete contraction $C^{l}, l \in \Theta_{\sigma-2} \backslash \Theta_{\sigma-2}^{*}$, we have that $C_{g}^{l}\left(\psi_{1}, \ldots, \psi_{s}\right)$, written in the form (3.44) does not have $s$ factors $\Delta \psi_{h}$ (by definition). Hence, each complete contraction $C^{f}, f \in F_{\sigma-2}^{2}$, in the decomposition of each $C_{g}^{l}\left(\psi_{1}, \ldots, \psi_{s}\right)$, $l \in \Theta_{\sigma-2} \backslash \Theta_{\sigma-2}^{*}$ must have $\delta \geq \mu+2$. This shows (3.43).

In view of (3.40), and by applying the same "by hand" technique as for equation (3.4), we see that we can find a vector field $\sum_{h \in H} a_{h} C_{g}^{h, i}\left(\psi_{1}, \ldots, \psi_{s}\right)$ so that modulo complete contractions of length $\geq \sigma+1$ :

$$
\begin{gathered}
\sum_{f \in F_{\sigma-2}^{2}} a_{f} C_{g}^{f}\left(\psi_{1}, \ldots, \psi_{s}\right)-\operatorname{div}_{i} \sum_{h \in H} a_{h} C_{g}^{h, i}\left(\psi_{1}, \ldots, \psi_{s}\right)=(\text { const })_{!} C_{g}^{!}\left(\psi_{1}, \ldots, \psi_{s}\right) \\
\quad+\sum_{u=1}^{\sigma-2}(\text { const })_{!, u} C_{g}^{!, u}\left(\psi_{1}, \ldots, \psi_{s}\right)+\sum_{q=1}^{\sigma-2} \sum_{w=1}^{q-1}(\mathrm{const})_{!,(q, w)} C_{g}^{!,(q, w)}\left(\psi_{1}, \ldots, \psi_{s}\right)
\end{gathered}
$$




$$
+\sum_{p \in P_{\sigma-2}^{2, \delta \geq \mu+2}} a_{p} C_{g}^{p}\left(\psi_{1}, \ldots, \psi_{s}\right)+\sum_{z \in Z^{\prime}} a_{z} C_{g}^{z}\left(\psi_{1}, \ldots, \psi_{s}\right)
$$

where $\sum_{p \in P_{\sigma-2}^{2, \delta \geq \mu+2}} a_{p} C_{g}^{p}\left(\psi_{1}, \ldots, \psi_{s}\right)$ stands for a linear combination of complete contractions in the form (1.4) with length $\sigma, s=\sigma-2,|\Delta|=\sigma-2$ and $q=1$ and also $\delta \geq \mu+2$. The "by hand" construction of the vector field for (3.45) is the same as the proof of equation (3.4), with the slight caveat that we now also have the complete contraction $C^{\text {spec }}$. In particular, since we only know that $\delta \geq \mu+1$ for $C^{\text {spec }}$, we have to check that we get no correction terms of the form (1.4) with $q=1$. But this follows by the form of $C^{\text {spec }}$, i.e. that we have two factors $\nabla^{(t)} R$, so we never have to introduce correction terms by virtue of the second Bianchi identity.

A study of the sublinear combination $\sum_{f \in F_{\sigma-2}^{1}} a_{f} C_{g}^{f}\left(\psi_{1}, \ldots, \psi_{s}\right)$. We claim that this sublinear combination will be of the form:

$$
\begin{aligned}
& \sum_{f \in F_{\sigma-2}^{1}} a_{f} C_{g}^{f}\left(\psi_{1}, \ldots, \psi_{s}\right)=\sum_{d \in D} a_{d} C_{g}^{d}\left(\psi_{1}, \ldots, \psi_{s}\right) \\
& \quad+(\text { const })_{\beta} C_{g}^{\beta}\left(\psi_{1}, \ldots, \psi_{s}\right)+(\text { const })_{\gamma} C_{g}^{\gamma}\left(\psi_{1}, \ldots, \psi_{s}\right),
\end{aligned}
$$

where $\sum_{d \in D} a_{d} C_{g}^{d}\left(\psi_{1}, \ldots, \psi_{s}\right)$ stands for a generic linear combination of complete contractions in the form (1.4) with length $\sigma, s=\sigma-2,|\Delta|=\sigma-2$ and $q=1$ and also $\delta \geq \mu+2$ (just like the linear combination $\sum_{p \in P_{\sigma-2}^{2, \delta \geq \mu+2}} a_{p} C_{g}^{p}\left(\psi_{1}, \ldots, \psi_{s}\right)$ in $\left.(3.45)\right)$, while $C_{g}^{\beta}\left(\psi_{1}, \ldots, \psi_{s}\right)$ is the complete contraction:

$$
\operatorname{contr}\left(\Delta^{\left(\frac{n}{2}-\sigma-2\right)} \nabla^{i l} R_{i j k l} \otimes \nabla^{j k} R \otimes \Delta \psi_{1} \otimes \cdots \otimes \Delta \psi_{s}\right),
$$

with $\delta=\frac{n}{2} \geq \mu+1$, and $C_{g}^{\gamma}\left(\psi_{1}, \ldots, \psi_{s}\right)$ is the complete contraction:

$$
\operatorname{contr}\left(\Delta^{\left(\frac{n}{2}-\sigma-2\right)} \nabla^{j k} R \otimes \nabla^{i l} R_{i j k l} \otimes \Delta \psi_{1} \otimes \cdots \otimes \Delta \psi_{s}\right),
$$

with $\delta=\frac{n}{2} \geq \mu+1$.

Observe that the sublinear combination

$$
\text { (const) }{ }_{\beta} C_{g}^{\beta}\left(\psi_{1}, \ldots, \psi_{s}\right)+(\text { const })_{\gamma} C_{g}^{\gamma}\left(\psi_{1}, \ldots, \psi_{s}\right)
$$

above is exclusively the contribution of the complete contraction $C^{\alpha}$ in $I^{s}$ to the linear combination $\sum_{f \in F_{\sigma-2}^{1}} a_{f} C_{g}^{f}\left(\psi_{1}, \ldots, \psi_{s}\right)$. (The fact that such contractions in the forms (3.47), (3.48) do arise in the decomposition of $C^{\alpha}$ can be directly checked.)

Now, we check that the contribution of all the other complete contractions in $I^{s}$ (i.e. other than $\left.C^{\alpha}\right)$ to the sublinear combination $\sum_{f \in F_{\sigma-2}^{1}} a_{f} C_{g}^{f}\left(\psi_{1}, \ldots, \psi_{s}\right)$ is indeed $\sum_{d \in D} a_{d} C_{g}^{d}\left(\psi_{1}\right.$, $\left.\ldots, \psi_{s}\right)$, as described in (3.46).

But this is straightforward to observe. We only need to check that each $C_{g}^{l}\left(\psi_{1}, \ldots, \psi_{s}\right)$ in the form (3.44), with $\delta \geq \mu$ and with less than $s$ factors $\Delta \psi_{s}$ can be written out as:

$$
C_{g}^{l}\left(\psi_{1}, \ldots, \psi_{s}\right)=\sum_{d \in D} a_{d} C_{g}^{d}\left(\psi_{1}, \ldots, \psi_{s}\right)+\sum_{q=0}^{2} \sum_{|\Delta| \leq \sigma-3} \sum_{f \in F_{|\Delta|}^{q}} a_{f} C_{g}^{f}\left(\psi_{1}, \ldots, \psi_{s}\right),
$$

where $\sum_{d \in D} a_{d} C_{g}^{d}\left(\psi_{1}, \ldots, \psi_{s}\right)$ is as above. (3.49) just follows by the decomposition of the Weyl tensor.

Now, we seek to "get rid" of the complete contractions $C^{\beta}, C^{\gamma}$. We construct vector fields $C^{\beta, i}, C^{\gamma, i}$, where $C^{\beta, i}$ arises from $C^{\beta}$ by erasing the index ${ }^{j}$ in the second factor and making the index $j$ that it contracted against in the first factor into a free index $i$. $C^{\gamma, i}$ arises from $C^{\gamma}$ 
by erasing the index ${ }^{j}$ in the first factor and making the index ${ }_{j}$ that it contracted against in the second factor into a free index $i$.

We then observe that modulo complete contractions of length $\geq \sigma+1$ :

$$
\begin{aligned}
& C_{g}^{\beta}\left(\psi_{1}, \ldots, \psi_{s}\right)-\operatorname{div}_{i} C_{g}^{\beta, i}\left(\psi_{1}, \ldots, \psi_{s}\right)=\sum_{z \in Z^{\prime}} a_{z} C_{g}^{z}\left(\psi_{1}, \ldots, \psi_{s}\right), \\
& C_{g}^{\gamma}\left(\psi_{1}, \ldots, \psi_{s}\right)-\operatorname{div}_{i} C_{g}^{\gamma, i}\left(\psi_{1}, \ldots, \psi_{s}\right)=\sum_{z \in Z^{\prime}} a_{z} C_{g}^{z}\left(\psi_{1}, \ldots, \psi_{s}\right) .
\end{aligned}
$$

Next, we claim that we can subtract a divergence $\sum_{h \in H} a_{h} C_{g}^{h, i}\left(\psi_{1}, \ldots, \psi_{s}\right)$ from the linear combination $\sum_{d \in D} a_{d} C_{g}^{d}\left(\psi_{1}, \ldots, \psi_{s}\right)$ so that:

$$
\begin{gathered}
\sum_{d \in D} a_{d} C_{g}^{d}\left(\psi_{1}, \ldots, \psi_{s}\right)-\operatorname{div}_{i} \sum_{h \in H} a_{h} C_{g}^{h, i}\left(\psi_{1}, \ldots, \psi_{s}\right)=(\text { const })_{?} \cdot C_{g}^{?}\left(\psi_{1}, \ldots, \psi_{s}\right) \\
\quad+\sum_{u=1}^{s}(\text { const })_{? ?, u} \cdot C_{g}^{? ?, u}\left(\psi_{1}, \ldots, \psi_{s}\right)+\sum_{z \in Z^{\prime}} a_{z} C_{g}^{z}\left(\psi_{1}, \ldots, \psi_{s}\right),
\end{gathered}
$$

where $C_{g}^{?}\left(\psi_{1}, \ldots, \psi_{s}\right)$ is the complete contraction:

$$
\operatorname{contr}\left(\Delta^{\left(\frac{n}{2}-\sigma-1\right)} \nabla^{i l} R_{i j k l} \otimes \operatorname{Ric}^{j k} \otimes \Delta \psi_{1} \otimes \cdots \otimes \Delta \psi_{s}\right),
$$

and it has $\delta \geq \mu+2$, while $C_{g}^{? ?, u}\left(\psi_{1}, \ldots, \psi_{s}\right)$ is the complete contraction:

$$
\operatorname{contr}\left(\Delta^{\left(\frac{n}{2}-\sigma-1\right)} \nabla^{i l} R_{i j k l} \otimes \nabla^{j k} \psi_{u} \otimes \Delta \psi_{1} \otimes \cdots \otimes \Delta \hat{\psi}_{u} \otimes \cdots \otimes \Delta \psi_{s}\right),
$$

and it has $\delta \geq \mu+2$. This follows by imitating the proof of the previous case. Then, we explicitly construct a vector field $\sum_{h \in H} a_{h} C_{g}^{h, i}\left(\psi_{1}, \ldots, \psi_{s}\right)$ so that:

$$
\begin{aligned}
& \text { (const) })_{?} \cdot C_{g}^{?}\left(\psi_{1}, \ldots, \psi_{s}\right)+\sum_{u=1}^{s}(\text { const })_{? ?, u} \cdot C_{g}^{? ?, u}\left(\psi_{1}, \ldots, \psi_{s}\right) \\
& -\operatorname{div}_{i} \sum_{h \in H} a_{h} C_{g}^{h, i}\left(\psi_{1}, \ldots, \psi_{s}\right)=(\text { const })_{\sharp} \cdot C_{g}^{\sharp}\left(\psi_{1}, \ldots, \psi_{s}\right)+\sum_{z \in Z^{\prime}} a_{z} C_{g}^{z}\left(\psi_{1}, \ldots, \psi_{s}\right),
\end{aligned}
$$

where $C_{g}^{\sharp}\left(\psi_{1}, \ldots, \psi_{s}\right)$ stands for the complete contraction:

$$
\operatorname{contr}\left(\Delta^{\left(\frac{n}{2}-\sigma-2\right)} \nabla^{i l} R_{i j k l} \otimes \nabla^{i^{\prime} l^{\prime}} R_{i^{\prime}}{ }^{j k}{ }_{l^{\prime}} \otimes \Delta \psi_{1} \otimes \cdots \otimes \Delta \psi_{s}\right),
$$

which has $\delta \geq \mu+1$.

Now, recall that by our lemma's assumption $L_{\mu}^{*}=\varnothing$, and $\sum_{j \in J^{*}} a_{j} C_{g}^{j}\left(\psi_{1}, \ldots, \psi_{s}\right)=$ (const) $)_{g}^{\prime \prime}\left(\psi_{1}, \ldots, \psi_{s}\right)$. Therefore, by our study of the three sublinear combinations $\sum_{f \in F_{\sigma-2}^{1}} \cdots, \sum_{f \in F_{\sigma-2}^{2}} \cdots, \sum_{f \in F_{\sigma-1}^{2}} \cdots$ we have shown that we can subtract a divergence from $I^{s}$ so that:

$$
\begin{aligned}
& I_{g}^{s}\left(\psi_{1}, \ldots, \psi_{s}\right)-\operatorname{div}_{i} \sum_{h \in H} a_{h} C_{g}^{h, i}\left(\psi_{1}, \ldots, \psi_{s}\right)=\sum_{l \in L_{\mu}} a_{l} C_{g}^{l}\left(\psi_{1}, \ldots, \psi_{s}\right) \\
& \quad+\sum_{j \in J \backslash J^{*}} a_{j} C_{g}^{j}\left(\psi_{1}, \ldots, \psi_{s}\right)+\sum_{q_{1}=0}^{2} \sum_{v=0}^{\sigma-3} \sum_{f \in F_{v}^{q_{1}}} a_{f} C_{g}^{f}\left(\psi_{1}, \ldots, \psi_{s}\right)+(\text { const }) ! \cdot C_{g}^{!}\left(\psi_{1}, \ldots, \psi_{s}\right) \\
& \quad+\sum_{y=1}^{s}(\mathrm{const})_{y, !} \cdot C_{g}^{y, *}\left(\psi_{1}, \ldots, \psi_{s}\right)+\sum_{w=1}^{s} \sum_{q=1}^{w-1}(\mathrm{const})_{w, q} \cdot C_{g}^{!, w, q}\left(\psi_{1}, \ldots, \psi_{s}\right)
\end{aligned}
$$




$$
+(\text { const })_{\sharp} C_{g}^{\sharp}\left(\psi_{1}, \ldots, \psi_{s}\right)+\sum_{z \in Z^{\prime}} a_{z} C_{g}^{z}\left(\psi_{1}, \ldots, \psi_{s}\right) .
$$

Here each $C^{l}, C^{j}$ has $|\Delta| \leq \sigma-3$. We then apply the exact same proof as in the third step in the previous subsection to show that $(\text { const })_{!}=0,(\text { const })_{y, !}=0$, const $)_{w, q}=0$, (const $)_{\sharp}=0$.

We have thus shown our claim in the case $s=\sigma-2, \mu<\frac{n}{2}$.

The proof of Lemmas $1.2,1.3,1.4$ when $s=\sigma-2, \sigma<\frac{n}{2}-1$ and $\mu=\frac{n}{2}$. In this case, we have that $\left.P(g)\right|_{\Theta_{\sigma-2}}$ can be written out as follows:

$$
\left.P(g)\right|_{\Theta_{\sigma-2}}=\sum_{l \in L} a_{l} C^{l}(g)
$$

where each $C^{l}(g)$ is a complete contraction in the form:

$$
\operatorname{contr}\left(\Delta^{\left(\alpha_{1}\right)} \nabla^{i l} W_{i j k l} \otimes \Delta^{\left(\alpha_{2}\right)} \nabla_{i^{\prime} l^{\prime}} W^{i^{\prime} j k l^{\prime}} \otimes \Delta^{\left(\alpha_{3}\right)} P_{a}^{a} \otimes \cdots \otimes \Delta^{\left(\alpha_{\sigma}\right)} P_{a}^{a}\right) .
$$

This is true by virtue of the fact that each $C^{l}(g), l \in \Theta_{\sigma-2}$ must have $\delta=\frac{n}{2}$. We assume with no loss of generality that the last $\sigma-2$ factors are arranged so that $\alpha_{3} \geq \alpha_{4} \geq \cdots \geq \alpha_{\sigma}$.

We denote by $L_{0} \subset L$ the index set of the complete contractions $C^{l}(g)$ with $\alpha_{3}=\cdots=\alpha_{\sigma}=0$. We claim that:

$$
\sum_{l \in L_{0}} a_{l} C^{l}(g)=0
$$

We observe that if we can show this, we will then have shown our claim. This is because every complete contraction $C^{l}(g), l \in L \backslash L_{0}$ will have a factor $\Delta^{(\alpha)} P_{a}^{a}$ with $\alpha>0$, hence each complete contraction $C^{l}, C^{j}, C^{f}$ in (1.5) will have a factor $\nabla^{(p)} \psi_{h}$ with $p \geq 3$ and two curvature factors that are not $R$ (scalar curvature). Hence, if we can show (3.51), we will have shown the remaining cases of both our Lemmas when $\mu=\frac{n}{2}$ and $\sigma<\frac{n}{2}-1$.

We will, in fact show a more general statement: We denote by $L_{\gamma} \subset L$ the subsets of complete contractions in the form with $\alpha_{3}=\gamma, \alpha_{4}=\cdots=\alpha_{\sigma}=0$. We will then show that for each $\gamma \geq 0$ :

$$
\sum_{l \in L_{\gamma}} a_{l} C^{l}(g)=0
$$

We show the above by an induction. Let us assume that (3.52) is known for every $\gamma>\gamma_{1}$. We will then show (3.52) for $\gamma=\gamma_{1}$. By our inductive assumption, we may cross out from $P(g)$ the sublinear combination $\sum_{\gamma>\gamma_{1}} \sum_{l \in L_{\gamma}} a_{l} C^{l}(g)$. Now, we consider $I_{g}^{s}\left(\psi_{1}, \ldots, \psi_{s}\right)$. We pick out the sublinear combination of $I_{g}^{s, \gamma_{1}}\left(\psi_{1}, \ldots, \psi_{s}\right)$ of complete contractions in the form:

$$
\operatorname{contr}\left(\Delta^{\left(\alpha_{1}\right)} \nabla^{i l} W_{i j k l} \otimes \Delta^{\left(\alpha_{2}\right)} \nabla_{i^{\prime} l^{\prime}} W^{i^{\prime} j k l^{\prime}} \otimes \Delta^{\left(\gamma_{1}+1\right)} \psi_{1} \otimes \Delta \psi_{2} \otimes \cdots \otimes \Delta \psi_{\sigma-2}\right)
$$

(we make the convention that $\alpha_{1} \geq \alpha_{2}$ ).

Clearly, if we can show that modulo complete contractions of length $\geq \sigma+1$ :

$$
I_{g}^{s, \gamma_{1}}\left(\psi_{1}, \ldots, \psi_{s}\right)=0,
$$

we will then have shown our inductive claim.

By the transformation law of the Schouten tensor under conformal re-scalings (see [3]), and by virtue of our inductive hypothesis (3.50) for $\gamma>\gamma_{1}$, we deduce that we can write out:

$$
I_{g}^{s}\left(\psi_{1}, \ldots, \psi_{s}\right)=I_{g}^{s, \gamma_{1}}\left(\psi_{1}, \ldots, \psi_{s}\right)+\sum_{u \in U_{1}} a_{u} C_{g}^{u}\left(\psi_{1}, \ldots, \psi_{s}\right)+\sum_{u \in U_{2}} a_{u} C_{g}^{u}\left(\psi_{1}, \ldots, \psi_{s}\right),
$$


where each $C^{u}$ is in the form (3.50), and for $u \in U_{1} \alpha_{h}>0$ for at least one $h>0$, while for each $u \in U_{2}$ we have that $\alpha_{3}<\gamma_{1}$.

We now use the silly divergence formula for $I_{g}^{s}$ (recall this formula from [1]), by integrating by parts with respect to the derivatives on the factor $\nabla^{(\alpha)} \psi_{1}$. We denote the silly divergence formula for $I^{s}$ by silly $\left[I_{g}^{s}\right]$. We focus on the sublinear combination silly ${ }_{*}\left[I_{g}^{s}\right]$ of complete contractions in silly $\left[I_{g}^{s}\right]$ in the form:

$$
\begin{aligned}
& \operatorname{contr}\left(\nabla^{t_{1} \ldots t_{\gamma_{1}+1}} \Delta^{\left(\alpha_{1}\right)} \nabla^{i l} W_{i j k l} \otimes \nabla_{t_{1} \ldots t_{\gamma_{1}+1}} \Delta^{\left(\alpha_{2}\right)} \nabla_{i^{\prime} l^{\prime}} W^{i^{\prime} j k l^{\prime}}\right. \\
& \left.\otimes \psi_{1} \otimes \Delta \psi_{2} \otimes \cdots \otimes \Delta \psi_{\sigma-2}\right) .
\end{aligned}
$$

Now, let us write out $I_{g}^{s, \gamma_{1}}\left(\psi_{1}, \ldots, \psi_{s}\right)$ in the form:

$$
I_{g}^{s, \gamma_{1}}\left(\psi_{1}, \ldots, \psi_{s}\right)=\sum_{(p, q)} a_{(p, q)} C_{g}^{(p, q)}\left(\psi_{1}, \ldots, \psi_{s}\right),
$$

where $C^{(p, q)}$ stands for the complete contraction in the form (3.53) with $\alpha_{1}=p, \alpha_{2}=q$. We then denote by $C_{g}^{(p, q), \sharp}$ the complete contraction that arises from $C^{(p, q)}$ by replacing the factor $\Delta^{\left(\gamma_{1}+1\right)} \psi_{1}$ by a factor $\psi_{1}$ (with no derivatives) and hitting the factor $\Delta^{(p)} \nabla^{i l} W_{i j k l}$ by derivatives $\nabla^{t_{1} \ldots t_{\gamma_{1}+1}}$ and the factor $\Delta^{(q)} \nabla_{i^{\prime} l^{\prime}} W^{i^{\prime} j k l^{\prime}}$ by derivatives $\nabla_{t_{1} \ldots t_{\gamma_{1}+1}}$ (we are using the Einstein summation convention). We then make three claims. Firstly:

$$
\text { silly }_{*}\left[I_{g}^{s}\right]=2^{\gamma_{1}} \sum_{(p, q)} a_{(p, q)} C_{g}^{(p, q), \sharp}\left(\psi_{1}, \ldots, \psi_{s}\right) .
$$

Now, for each $C^{(p, q), \sharp}$ above, we denote by $C_{g}^{(p, q), \sharp, \iota}\left(\psi_{1}, \ldots, \psi_{s}\right)$ the complete contraction that arises from it by replacing the two factors $\nabla^{t_{1} \ldots t_{\gamma_{1}+1}} \Delta^{\left(\alpha_{1}\right)} \nabla^{i l} W_{i j k l}, \nabla_{t_{1} \ldots t_{\gamma_{1}+1}} \Delta^{\left(\alpha_{2}\right)} \nabla_{i^{\prime} l^{\prime}} W^{i^{\prime} j k l^{\prime}}$ by factors $\nabla^{t_{1} \ldots t_{\gamma_{1}+1}} \Delta^{\left(\alpha_{1}\right)} \nabla^{i l} R_{i j k l}, \nabla_{t_{1} \ldots t_{\gamma_{1}+1}} \Delta^{\left(\alpha_{2}\right)} \nabla_{i^{\prime} l^{\prime}} R^{i^{\prime} j k l^{\prime}}$. Our second claim is that modulo complete contractions of length $\geq \sigma+1$ :

$$
\sum_{(p, q)} a_{(p, q)} C_{g}^{(p, q), \sharp, \iota}\left(\psi_{1}, \ldots, \psi_{s}\right)=0 .
$$

Our third claim is that from the above we can deduce that each $a_{(p, q)}=0$. If we can show the above three claims, then clearly (3.52) with $\gamma=\gamma_{1}$ will follow.

We begin with our first claim. For each complete contraction $C_{g}\left(\psi_{1}, \ldots, \psi_{s}\right)$ in $(3.54)$, we denote by $\operatorname{sil}\left[C_{g}\left(\psi_{1}, \ldots, \psi_{s}\right)\right] \cdot \psi_{1}$ the sublinear combination of complete contractions in the right hand side of silly $\left[I_{g}^{s}\right]$. This notation extends to linear combinations. The silly divergence formula just tells us that:

$$
\operatorname{sil}\left[I_{g}^{s, \gamma_{1}}\left(\psi_{1}, \ldots, \psi_{s}\right)\right]+\sum_{u \in U_{1}} a_{u} \operatorname{sil}\left[C_{g}^{u}\left(\psi_{1}, \ldots, \psi_{s}\right)\right]+\sum_{u \in U_{2}} a_{u} \operatorname{sil}\left[C_{g}^{u}\left(\psi_{1}, \ldots, \psi_{s}\right)\right]=0
$$

If we can show that for $u \in U_{1} \bigcup U_{2}, \operatorname{sil}\left[C_{g}^{u}\right]$ contains no complete contractions in the form (3.55), our first claim will then follow.

This claim is trivial for the complete contractions $C_{g}^{u}, u \in U_{1}$. We notice that if $C_{g}^{u}$ has a factor $\Delta^{(\alpha)} \psi_{h}, \alpha>1, h>1$, then each complete contraction in $\operatorname{sil}\left[C_{g}^{u}\right]$ will have a factor $\nabla^{(p)} \psi_{h}, p \geq 2 \alpha>2$. On the other hand, for each $u \in U_{2}$, we observe that each complete contraction in $\operatorname{sil}\left[C_{g}^{u}\right]$ will have less than $\gamma_{1}+3$ indices in the first factor $\nabla^{(m)} W_{i j k l}$ contracting against indices in the second factor $\nabla^{\left(m^{\prime}\right)} W_{i j k l}$. So we indeed see that $\operatorname{sil}\left[C_{g}^{u}\right], u \in U_{2}$ has no complete contraction of the form (3.55). Therefore we have shown our first claim. 
Now, we check the second claim. We write out silly $\left[I_{g}^{s}\left(\psi_{1}, \ldots, \psi_{s}\right)\right]$ as a linear combination of complete contractions in the form (1.4). We define silly spec $_{[}\left[I_{g}^{s}\left(\psi_{1}, \ldots, \psi_{s}\right)\right]$ to stand for the sublinear combination of complete contractions with $s-1$ factors $\Delta \psi_{2}, \ldots, \Delta \psi_{s}$, one factor $\psi_{1}$ and two factors $\nabla^{(m)} R_{i j k l}, \nabla^{\left(m^{\prime}\right)} R_{i^{\prime} j^{\prime} k^{\prime} l^{\prime}}$ with $\gamma_{1}+3$ particular contractions between them. (We write $\nabla^{(m)} R_{i j k l}, \nabla^{\left(m^{\prime}\right)} R_{i^{\prime} j^{\prime} k^{\prime} l^{\prime}}$ but there is no restriction on the particular contractions of the indices $i,{ }_{j}, k, l, i^{\prime}, j^{\prime}, k^{\prime}, l^{\prime}$, i.e. we can have ${ }_{i}, k$ contracting between themselves etc.) Since the silly divergence formula must hold formally, we deduce that:

$$
\text { silly }_{\text {spec }}\left[I_{g}^{s}\left(\psi_{1}, \ldots, \psi_{s}\right)\right]=0 \text {, }
$$

modulo complete contractions of length $\geq \sigma+1$.

Moreover, clearly any complete contraction $C_{g}\left(\psi_{1}, \ldots, \psi_{s}\right)$ in the form (3.50) with either a factor $\nabla^{(p)} \psi_{h}, p \geq 3$ or with less than $\gamma_{1}+3$ pairs of particular contractions between the first two factors $\nabla^{(m)} W_{i j k l}, \nabla^{\left(m^{\prime}\right)} W_{i j k l}$ cannot contribute to the sublinear combination silly $_{\text {spec }}\left[I_{g}^{s}\left(\psi_{1}, \ldots, \psi_{s}\right)\right]$. Hence, we derive that $\operatorname{sil}\left[C_{g}^{u}\right], u \in U_{1} \bigcup U_{2}$ do not contribute to the sublinear combination silly ${ }_{\text {spec }}\left[I_{g}^{s}\right]$, whereas by virtue of the decomposition of the factor $\nabla^{i l} W_{i j k l}$, we have that:

$$
\text { silly }_{\text {spec }}\left[I_{g}^{s}\left(\psi_{1}, \ldots, \psi_{s}\right)\right]=\sum_{(p, q)} 2^{\gamma_{1}+1} \frac{n-3}{n-2} a_{(p, q)} C_{g}^{(p, q), \sharp, \iota}\left(\psi_{1}, \ldots, \psi_{s}\right)+\sum_{t \in T} a_{t} C_{g}^{t}\left(\psi_{1}, \ldots, \psi_{s}\right),
$$

where each is in the form (1.4) with either two factors of the form $\nabla^{(p)} R, \nabla^{\left(p^{\prime}\right)} R,{ }^{33}$ or one factor of the form $\nabla^{(p)} R$ and one factor of the form $\nabla^{(m)} R_{i j k l}$ where none of the indices ${ }_{i},{ }_{j}, k, l$ are contracting between themselves. In fact, in order to distinguish these two cases we break the index set $T$ into subsets $T_{1}, T_{2}$ accordingly.

Now, since (3.56) holds formally we deduce that modulo complete contractions of length $\geq \sigma+1$ :

$$
\sum_{t \in T_{1}} a_{t} C_{g}^{t}\left(\psi_{1}, \ldots, \psi_{s}\right)=0
$$

Then, again since (3.56) holds formally, we deduce that modulo complete contractions of length $\geq \sigma+1$ :

$$
\sum_{t \in T_{2}} a_{t} C_{g}^{t}\left(\psi_{1}, \ldots, \psi_{s}\right)=0
$$

Therefore, we have shown that modulo complete contractions of length $\geq \sigma+1$ :

$$
\sum_{(p, q)} a_{(p, q)} C_{g}^{(p, q), \sharp, \iota}\left(\psi_{1}, \ldots, \psi_{s}\right)=0 .
$$

Clearly, since the above holds formally, we can deduce that for each different pair $(p, q)$ we must have:

$$
a_{(p, q)} C_{g}^{(p, q), \sharp, \iota}\left(\psi_{1}, \ldots, \psi_{s}\right)=0,
$$

and since each complete contraction $C_{g}^{(p, q), \sharp, \iota}\left(\psi_{1}, \ldots, \psi_{s}\right)$ is not identically the zero contraction, we deduce that each $a_{(p, q)}$ must be zero. This was our third claim. We have thus shown our inductive statement, and we have proven our claim in the case $s=\sigma-2, \sigma<\frac{n}{2}-1, \mu=\frac{n}{2}$.

The proof of Lemmas 1.2, 1.3, 1.4 when $s=\sigma-2$ and $\sigma=\frac{n}{2}-1$. We observe that any complete contraction $C^{l}(g)$ in $\left.P(g)\right|_{\sigma}$, when $\sigma=\frac{n}{2}-1$ must be in the form:

$$
\operatorname{contr}\left(\nabla^{(m)} W_{i j k l} \otimes \nabla^{\left(m^{\prime}\right)} W_{i^{\prime} j^{\prime} k^{\prime} l^{\prime}} \otimes \nabla^{\left(p_{1}\right)} P_{a b} \otimes \cdots \otimes \nabla^{\left(p_{\frac{n}{2}-3}\right)} P_{a^{\prime} b^{\prime}}\right)
$$

must have exactly two derivatives (i.e. $m+m^{\prime}+p_{1}+\cdots+p_{\frac{n}{2}-3}=2$ ) and moreover $\delta \leq \frac{n}{2}-1 .^{34}$

\footnotetext{
${ }^{33}$ Recall that $R$ here is the scalar curvature.

${ }^{34}$ It follows easily that in this case $\mu \leq \frac{n}{2}$.
} 
We distinguish two cases. Either $\mu=\frac{n}{2}-1$ or $\mu<\frac{n}{2}-1$. We start with the first case. Then we see that:

$$
\left.P(g)\right|_{\Theta_{\sigma-2}}=(\text { const }) \cdot C(g),
$$

where $C(g)$ is the complete contraction:

$$
\operatorname{contr}\left(\nabla^{l} W_{i j k l} \otimes \nabla_{l^{\prime}} W^{i j k l^{\prime}} \otimes\left(P_{a}^{a}\right)^{\sigma-2}\right) .
$$

We will show that (const) $=0$. This follows easily by the silly divergence formula. We consider $I_{g}^{s}\left(\psi_{1}, \ldots, \psi_{s}\right)$. It follows that:

$$
I_{g}^{s}\left(\psi_{1}, \ldots, \psi_{s}\right)=s ! \cdot C_{g}\left(\psi_{1}, \ldots, \psi_{s}\right),
$$

where $C_{g}\left(\psi_{1}, \ldots, \psi_{s}\right)$ is the contraction:

$$
\operatorname{contr}\left(\nabla^{l} W_{i j k l} \otimes \nabla_{l^{\prime}} W^{i j k l^{\prime}} \otimes \Delta \psi_{1} \otimes \cdots \otimes \Delta \psi_{\sigma-2}\right) .
$$

We consider silly $\left[I_{g}^{s}\right]$, with integration by parts with respect to $\nabla^{(p)} \psi_{1}$, and we focus on the sublinear combination:

$$
\text { silly }_{+}\left[I_{g}^{s}\left(\psi_{1}, \ldots, \psi_{s}\right)=(\text { const })^{\prime} \cdot \operatorname{contr}\left(\nabla^{l a} W_{i j k l} \otimes \nabla_{l^{\prime} a} W^{i j k l^{\prime}} \otimes \psi_{1} \otimes \Delta \psi_{2} \otimes \cdots \otimes \Delta \psi_{\sigma-2}\right) .\right.
$$

It follows by the same arguments as before that silly ${ }_{+}\left[I_{g}^{s}\right]=0$, modulo complete contractions of length $\geq \sigma+1$ and that (const) ${ }^{\prime}=2 \cdot s !$ (const), and hence we deduce our claim in the case $\mu=\frac{n}{2}-1$.

Now, the case $\mu<\frac{n}{2}-1$. We notice that in this case, the sublinear combination of complete contractions $C^{l}(g), l \in \Theta_{\sigma-2}$ that have $\sigma-2$ factors $P_{a}^{a}$ is precisely of the form (const) $C(g)$, where $C(g)$ is in the form (3.57) (since $\left.P(g)\right|_{\Theta_{\sigma-1}}$ is assumed "good"). We can then prove our claim in this case by exactly applying the method of the case $\sigma=\frac{n}{2}-1$ when $s<\sigma-2$.

\subsection{The proof of Lemmas 1.2, 1.3, 1.4 when $s=\sigma-1$, and Lemma 1.5 when $s=\sigma-1$}

In this case, we follow the same pattern as in the two previous ones. We begin by a trivial observation regarding the maximum value that $\mu$ can have ${ }^{35}$. By virtue of the formula $I_{g}^{\sigma-1}(\phi):=$ $\left.\frac{d^{\sigma-1}}{d t^{\sigma-1}}\right|_{t=0} e^{n t \phi} P\left(e^{2 t \phi} g\right),{ }^{36}$ and since we observe that any complete contraction in the form (1.3) with length $\sigma$ and $\sigma-1$ factors $P_{a}^{a}$ must be zero, modulo complete contractions of length $\geq \sigma+1$, we observe that necessarily $\mu \leq \frac{n}{2}-1$ in this case.

We again distinguish the two cases $\mu=\frac{n}{2}-1$ and $\mu<\frac{n}{2}-1$. We start with the second case.

Proof of Lemma 1.5 when $s=\sigma-1, \mu<\frac{n}{2}-1$. We focus on the sublinear combination of complete contractions $C^{l}(g)$ in $\left.P(g)\right|_{\Theta_{\sigma-1}}$ that have $\sigma-2$ factors $P_{a}^{a}$. We denote their index set by $\Theta_{\sigma-1}^{+} \subset \Theta_{\sigma-1}$. By the usual "manual" construction of explicit divergences it follows that we can subtract a divergence $\operatorname{div}_{i} \sum_{h \in H} a_{h} C^{h, i}(g)$ from $\left.P(g)\right|_{\Theta_{\sigma-1}^{+}}$so that modulo complete contractions of length $\geq \sigma+1$ :

$$
\left.P(g)\right|_{\Theta_{\sigma-1}^{+}}-\operatorname{div}_{i} \sum_{h \in H} a_{h} C^{h, i}(g)=(\text { const })_{V} \cdot C^{V}(g)+\sum_{t \in T} a_{t} C^{t}(g)+\sum_{y \in Y} a_{y} C^{y}(g),
$$

\footnotetext{
${ }^{35}$ Recall that $\mu$ stands for the minimum value of $\delta=\delta_{W}+\delta_{P}$ among the complete contractions $C^{l}(g), l \in \Theta_{s}$.

${ }^{36}$ Thus in particular the terms of length $\sigma-1$ in $I_{g}^{\sigma-1}(\phi)$ arise from the sublinear combination $P(g) \Theta_{\sigma-1}$ by just replacing each factor $\nabla_{r_{1} \ldots r_{a}}^{(a)} P_{i j}$ by $-\nabla_{r_{1} \ldots r_{a} i j}^{(a+2)} \phi$.
} 
where $C^{V}(g)$ is the complete contraction:

$$
\operatorname{contr}\left(\Delta^{\frac{n}{2}-(\sigma-2)-3} \nabla^{i l} W_{i j k l} \otimes P^{j k} \otimes\left(P_{a}^{a}\right)^{\sigma-2}\right),
$$

and where each $C^{y}(g)$ is in the form (1.3) with at least two factors $\nabla^{(m)} W_{i j k l}$. Also, each $C^{t}(g)$ is in the form (1.3) with $\delta \geq \mu$ and also with less than $\sigma-2$ factors $P_{a}^{a}$.

Now, we observe that $C^{V}(g)$ has $\delta=\frac{n}{2}-1 \geq \mu+1$. Therefore, if we define $C^{V, i}(g)$ to stand for the vector field that arises from $C^{V}(g)$ by erasing ${ }^{i}$ and making $i$ in the first factor into a free index, we will then have:

$$
C^{V}(g)-\operatorname{div}_{i} C^{V, i}(g)=\sum_{y \in Y} a_{y} C^{y}(g)+\sum_{d \in D} a_{d} C^{d}(g) .
$$

Here each $C^{y}(g)$ is as above, while each $C^{d}(g)$ has $\delta \geq \mu$ and also has strictly less than $\sigma-2$ factors $P_{a}^{a}$. Thus, by subtracting the divergence $\operatorname{div}_{i}\left[\sum_{h \in H} a_{h} C^{h, i}(g)+(\text { const })_{V} \cdot C^{V, i}(g)\right]$ from $P(g)$, we may assume with no loss of generality that $\left.P(g)\right|_{\Theta_{\sigma-1}}$ contains only complete contractions with at most $\sigma-3$ factors $P_{a}^{a}$. We will be using this fact below.

Proof of Lemmas 1.2, 1.3, 1.4 when $s=\boldsymbol{\sigma}-\mathbf{1}, \boldsymbol{\mu}<\frac{\boldsymbol{n}}{\mathbf{2}}-\mathbf{1}$. We focus on $I_{g}^{s}\left(\psi_{1}, \ldots, \psi_{s}\right) ;^{37}$ for the global conformal invariants $P(g)$ above, and we decompose the Weyl tensors (i.e. we write $I_{g}^{s}$ as a linear combination in the form (1.5)). In the notation of the introduction, we have that $F^{*}=F_{\sigma-1}^{1} \bigcup F_{\sigma-2}^{1}$.

We first focus our attention on the sublinear combination $F_{\sigma-1}^{1}$. As in the previous cases, it follows that we can write out:

$$
\sum_{f \in F_{\sigma-1}^{1}} a_{f} C_{g}^{f}\left(\psi_{1}, \ldots, \psi_{s}\right)=(\mathrm{const})_{+} \cdot C_{g}^{+}\left(\psi_{1}, \ldots, \psi_{s}\right)+\sum_{u=1}^{s} a_{u} C_{g}^{u}\left(\psi_{1}, \ldots, \psi_{s}\right),
$$

where $C_{g}^{+}\left(\psi_{1}, \ldots, \psi_{s}\right)$ stands for the complete contraction:

$$
\operatorname{contr}\left(\Delta^{\left(\frac{n}{2}-\sigma\right)} R \otimes \Delta \psi_{1} \otimes \cdots \otimes \Delta \psi_{s}\right),
$$

while $C_{g}^{u}\left(\psi_{1}, \ldots, \psi_{s}\right)$ stands for the complete contraction:

$$
\operatorname{contr}\left(\Delta^{\left(\frac{n}{2}-\sigma+1\right)} \psi_{u} \otimes \Delta \psi_{1} \otimes \ldots \Delta \hat{\psi}_{u} \cdots \otimes \Delta \psi_{s} \otimes R\right) .
$$

We observe that each of the above complete contraction has $\delta=\frac{n}{2}+1 \geq \mu+2$. As before, we can explicitly construct a divergence $\operatorname{div}_{i} \sum_{h \in H} a_{h} C_{g}^{h, i}\left(\psi_{1}, \ldots, \psi_{s}\right)$ such that:

$$
\begin{aligned}
& \sum_{f \in F_{\sigma-1}^{1}} a_{f} C_{g}^{f}\left(\psi_{1}, \ldots, \psi_{s}\right)-\operatorname{div}_{i} \sum_{h \in H} a_{h} C_{g}^{h, i}\left(\psi_{1}, \ldots, \psi_{s}\right)=\sum_{u=1}^{s}(\text { const })_{*, u} C_{g}^{*, u}\left(\psi_{1}, \ldots, \psi_{s}\right) \\
& \quad+\sum_{q=1}^{s} \sum_{w=1}^{q-1}(\mathrm{const})_{*,(q, w)} C_{g}^{*,(q, w)}\left(\psi_{1}, \ldots, \psi_{s}\right)+\sum_{z \in Z^{\prime}} a_{z} C_{g}^{z}\left(\psi_{1}, \ldots, \psi_{s}\right),
\end{aligned}
$$

where $C_{g}^{*, u}\left(\psi_{1}, \ldots, \psi_{s}\right)$ stands for the complete contraction:

$$
\operatorname{contr}\left(\Delta^{\left(\frac{n}{2}-\sigma-1\right)} R \otimes \Delta^{(2)} \psi_{u} \otimes \Delta \psi_{1} \otimes \cdots \otimes \Delta \hat{\psi}_{u} \otimes \cdots \otimes \Delta \psi_{s}\right),
$$

\footnotetext{
${ }^{37}$ Recall that $I_{g}^{s}(\phi):=\left.\frac{d^{s}}{d t^{s}}\right|_{t=0}\left(e^{n t \phi} P^{2 t \phi} g\right)$ and that $I_{g}^{s}\left(\psi_{1}, \ldots, \psi_{s}\right)$ arises from $I_{g}^{s}(\phi)$ by just polarizing the function $\phi$.
} 
while $C_{g}^{*,(q, w)}\left(\psi_{1}, \ldots, \psi_{s}\right)$ stands for the complete contraction

$$
\operatorname{contr}\left(\Delta^{\left(\frac{n}{2}-\sigma\right)} \psi_{q} \otimes \Delta^{(2)} \psi_{w} \otimes \Delta \psi_{1} \otimes \cdots \otimes \Delta \hat{\psi}_{q} \otimes \cdots \otimes \Delta \hat{\psi}_{w} \otimes \cdots \otimes \Delta \psi_{s}\right),
$$

and $\sum_{z \in Z^{\prime}} a_{z} C_{g}^{z}\left(\psi_{1}, \ldots, \psi_{s}\right)$ stands for a generic linear combination of complete contractions with $|\Delta| \leq \sigma-3$ and $\delta \geq \mu+1$.

We next focus our attention on the sublinear combination $\sum_{f \in F_{\sigma-2}^{1}} a_{f} C_{g}^{f}\left(\psi_{1}, \ldots, \psi_{s}\right)$. Since $\left.P(g)\right|_{\Theta_{\sigma-1}}$ has no complete contractions with $\sigma-2$ factors $P_{a}^{a}$, it follows that any $C_{g}^{f}\left(\psi_{1}, \ldots, \psi_{s}\right)$, $f \in F_{\sigma-2}^{1}$ must have $\delta \geq \mu+2$. Therefore, as in the previous subsection, we can construct a vector field $\sum_{h \in H} a_{h} C_{g}^{h, i}\left(\psi_{1}, \ldots, \psi_{s}\right)$ so that, modulo complete contractions of length $\geq \sigma+1$ :

$$
\begin{aligned}
& \sum_{f \in F_{\sigma-2}^{1}} a_{f} C_{g}^{f}\left(\psi_{1}, \ldots, \psi_{s}\right)-\sum_{h \in H} a_{h} C_{g}^{h, i}\left(\psi_{1}, \ldots, \psi_{s}\right)=\sum_{u=1}^{s}(\text { const })_{*, u}^{\prime} C_{g}^{*, u}\left(\psi_{1}, \ldots, \psi_{s}\right) \\
& +\sum_{q=1}^{s} \sum_{w=1}^{q-1}(\mathrm{const})_{*,(q, w)}^{\prime} C_{g}^{*,(q, w)}\left(\psi_{1}, \ldots, \psi_{s}\right)+\sum_{b \in B} a_{b} C_{g}^{b}\left(\psi_{1}, \ldots, \psi_{s}\right)+\sum_{z \in Z^{\prime}} a_{z} C_{g}^{z}\left(\psi_{1}, \ldots, \psi_{s}\right) .
\end{aligned}
$$

Here the complete contractions $C^{*, u}, C^{*,(q, w)}$ are the same as above, while $\sum_{b \in B} a_{b} C_{g}^{b}\left(\psi_{1}, \ldots, \psi_{s}\right)$ stands for a linear combination of complete contractions in the form (1.4) with length $\sigma, \delta \geq \mu+2$, $q=0$ and $|\Delta|=\sigma-2$.

Finally, we focus on the sublinear combinations in $I_{g}^{s}\left(\psi_{1}, \ldots, \psi_{s}\right)$ which have $q=0$ (i.e. we consider the complete contractions that are indexed in the sets $L_{\mu}, J$ in $\left.(1.5)^{38}\right)$. Now, since we have that each $C^{l}(g), l \in \Theta_{\sigma-1}$ has less than $\sigma-2$ factors $P_{a}^{a}$, it follows that all the complete contractions in $L_{\mu}, J$ in (1.5) must have $|\Delta| \leq \sigma-3$.

Then (by the usual construction), we can construct a linear combination of vector fields, $\sum_{h \in H} a_{h} C_{g}^{h, i}\left(\psi_{1}, \ldots, \psi_{s}\right)$ so that, modulo complete contractions of length $\geq \sigma+1$ :

$$
\begin{aligned}
\sum_{b \in B} a_{b} C_{g}^{b}\left(\psi_{1}, \ldots, \psi_{s}\right)-\operatorname{div}_{i} \sum_{h \in H} a_{h} C_{g}^{h, i}\left(\psi_{1}, \ldots, \psi_{s}\right) & \\
= & \sum_{u=1}^{s}(\text { const })_{L, u} C_{g}^{L, u}\left(\psi_{1}, \ldots, \psi_{s}\right)+\sum_{z \in Z^{\prime}} a_{z} C_{g}^{z}\left(\psi_{1}, \ldots, \psi_{s}\right),
\end{aligned}
$$

where $C_{g}^{L, u}\left(\psi_{1}, \ldots, \psi_{s}\right)$ is the complete contraction:

$$
\operatorname{contr}\left(\Delta^{\left(\frac{n}{2}-(\sigma-2)-3\right)} \nabla^{i l} R_{i j k l} \otimes \nabla^{j k} \psi_{u} \otimes \Delta \psi_{1} \otimes \cdots \otimes \Delta \hat{\psi}_{u} \otimes \cdots \otimes \Delta \psi_{s}\right),
$$

which has $\delta \geq \mu+2$, and where $\sum_{z \in Z^{\prime}} a_{z} C_{g}^{z}\left(\psi_{1}, \ldots, \psi_{s}\right)$ is as above.

Moreover, we define $C_{g}^{L, u, i}\left(\psi_{1}, \ldots, \psi_{s}\right)$ to stand for the vector field that arises from $C_{g}^{L, u}\left(\psi_{1}\right.$, $\left.\ldots, \psi_{s}\right)$ by erasing the index ${ }^{i}$ and making $i$ into a free index. We then observe that, modulo complete contractions of length $\geq \sigma+1$ :

$$
C_{g}^{L, u}\left(\psi_{1}, \ldots, \psi_{s}\right)-\operatorname{div}_{i} C_{g}^{L, u, i}\left(\psi_{1}, \ldots, \psi_{s}\right)=\sum_{z \in Z^{\prime}} a_{z} C_{g}^{z}\left(\psi_{1}, \ldots, \psi_{s}\right) .
$$

In conclusion, we have shown that we can subtract a linear combination of divergences from $I_{g}^{s}\left(\psi_{1}, \ldots, \psi_{s}\right)$ so that, modulo complete contractions of length $\geq \sigma+1$ :

$$
I_{g}^{s}\left(\psi_{1}, \ldots, \psi_{s}\right)-\operatorname{div}_{i} C_{g}^{h, i}\left(\psi_{1}, \ldots, \psi_{s}\right)=\sum_{l \in L_{\mu}} a_{l} C_{g}^{l, \iota}\left(\psi_{1}, \ldots, \psi_{s}\right)+\sum_{j \in J} a_{j} C_{g}^{j}\left(\psi_{1}, \ldots, \psi_{s}\right)
$$

\footnotetext{
${ }^{38}$ These sets index the complete contractions in $I_{g}^{s}$ with no factors $\nabla^{(p)}$ Ric.
} 


$$
\begin{aligned}
& +\sum_{f \in F^{1} \backslash\left(F_{\sigma-1}^{1} \cup F_{\sigma-2}^{1}\right)} a_{f} C_{g}^{f}\left(\psi_{1}, \ldots, \psi_{s}\right)+\sum_{u=1}^{s}(\text { const })_{*, u} C_{g}^{*, u}\left(\psi_{1}, \ldots, \psi_{s}\right) \\
& +\sum_{q=1}^{s} \sum_{w=1}^{q-1}(\mathrm{const})_{*,(q, w)} C_{g}^{*,(q, w)}\left(\psi_{1}, \ldots, \psi_{s}\right)+\sum_{z \in Z^{\prime}} a_{z} C_{g}^{z}\left(\psi_{1}, \ldots, \psi_{s}\right) .
\end{aligned}
$$

(The terms in the second line are not generic linear combinations - they stand for the original linear combinations in $I_{g}^{s}\left(\psi_{1}, \ldots, \psi_{s}\right)$ as in (1.5).)

Then, using the super divergence formula as in the previous subsection, we deduce that (const) $)_{, u}=0$ for every $u=1, \ldots, s$ and $(\text { const })_{*,(q, w)}=0$ for every $q=1, \ldots, s, w=1, \ldots, q-1$.

Therefore, we have proven Lemmas 1.1, 1.3, 1.4 when $s=\sigma-1$ and $\mu<\frac{n}{2}-1$.

Now, we consider the subcase where $\mu=\frac{n}{2}-1$. We see that in this case (modulo introducing correction terms with length $\sigma$ and two factors $\left.\nabla^{(m)} W_{i j k l}\right)$, we can write out $\left.P(g)\right|_{\Theta_{\sigma-1}}$ :

$$
\left.P(g)\right|_{\Theta_{\sigma-1}}=\sum_{\gamma \in \Gamma} a_{\gamma} C_{g}^{\gamma}\left(\psi_{1}, \ldots, \psi_{s}\right)+\sum_{\epsilon \in E} a_{\epsilon} C_{g}^{\epsilon}\left(\psi_{1}, \ldots, \psi_{s}\right),
$$

where each $C^{\gamma}(g)$ is in the form:

$$
\operatorname{contr}\left(\Delta^{(\alpha)} \nabla^{i l} W_{i j k l} \otimes \Delta^{(\beta)} P^{j k} \otimes \Delta^{\left(\rho_{1}\right)} P_{a}^{a} \otimes \cdots \otimes \Delta^{\left(\rho_{\sigma-2}\right)} P_{a}^{a}\right)
$$

(we are making the convention that $\rho_{1} \geq \cdots \geq \rho_{\sigma-2}$ ), while each $C^{\epsilon}(g)$ is in the form:

$$
\operatorname{contr}\left(\Delta^{(\alpha)} \nabla^{i l} W_{i j k l} \otimes \Delta^{\left(\beta_{1}\right)} \nabla^{t} P_{t}^{j} \otimes \Delta^{\left(\beta_{2}\right)} \nabla^{w} P_{w}^{k} \otimes \Delta^{\left(\rho_{1}\right)} P_{a}^{a} \otimes \cdots \otimes \Delta^{\left(\rho_{\sigma-3}\right)} P_{a}^{a}\right)
$$

(we are making the convention that $\rho_{1} \geq \cdots \geq \rho_{\sigma-3}$ ).

Now, a small observation. For each $C^{\gamma}(g)$ with $\beta>0$, we can construct "by hand" a vector field $C^{\gamma, i}(g)$ so that:

$$
C^{\gamma}(g)-\operatorname{div}_{i} C^{\gamma, i}(g)=\sum_{y \in Y} a_{y} C^{y}(g)+\sum_{t \in T} a_{t} C^{t}(g),
$$

where each $C^{y}(g)$ is a complete contraction in the form (3.59) and $\sum_{t \in T} a_{t} C^{t}(g)$ is a linear combination of complete contractions in the form (1.3) with at least two factors $\nabla^{(m)} W_{i j k l}$. The above holds modulo complete contractions of length $\geq \sigma+1$.

In view of (3.60), we can assume that each $C^{\gamma}(g)$ in $\left.P(g)\right|_{\Theta_{\sigma-1}}$ has $\beta=0$.

We then define $\Gamma_{\kappa}$ to stand for the index set of the complete contractions in the form (3.58) with $\beta=0, \rho_{1}=\kappa \geq 0$ and $\rho_{2}, \ldots, \rho_{\sigma-2}=0$. We observe that, by definition:

$$
\sum_{\gamma \in \Gamma_{\kappa}} a_{\gamma} C^{\gamma}(g)=(\text { const })_{\kappa} \cdot C^{\kappa}(g)
$$

where $C^{\kappa}(g)$ is in the form (3.58) with $\beta=0, \rho_{1}=\kappa, \rho_{2}=\cdots=\rho_{s-2}=0$ and with $\alpha=$ $\frac{n}{2}-(\sigma-2)-3$.

We will show that under the above assumptions, for each $\kappa \geq 0$ :

$$
\sum_{\gamma \in \Gamma_{\kappa}} a_{\gamma} C_{g}^{\gamma}\left(\psi_{1}, \ldots, \psi_{s}\right)=0
$$

modulo complete contractions of length $\geq \sigma+1$.

If we can show the above, we then see that we will have shown Lemmas 1.1, 1.3, 1.4 in this setting. This is true since if we consider any $P(g)$ with $\left.P(g)\right|_{\Theta_{\sigma-1}}$ that satisfies (3.61) 
and we then write out $I_{g}^{s}\left(\psi_{1}, \ldots, \psi_{s}\right)$ by decomposing the Weyl tensors, then each complete contraction $C^{l, \iota}, C^{f}, C^{j}$ in $I^{s}$ must have at least two factors $\nabla^{(t)} \psi_{h}$ with $t \geq 3$ and at least one factor either in the form $\nabla^{(m)} R_{i j k l}, m \geq 2$ or in the form $\nabla^{(p)}$ Ric, $p \geq 2$.

We show (3.61) by an induction. We assume that (3.61) holds for each $\kappa>\kappa_{1}$ and we will show it for $\kappa=\kappa_{1}$. We consider the silly divergence formula for $I_{g}^{s}\left(\psi_{1}, \ldots, \psi_{s}\right)$ - we denote it by silly $\left[I_{g}^{s}\left(\psi_{1}, \ldots, \psi_{s}\right)\right]-$ and we focus on the sublinear combination silly ${ }_{*}\left[I_{g}^{s}\right]$ that consists of the complete contractions in the form:

$$
\operatorname{contr}\left(\nabla_{t_{1} \ldots t_{\kappa_{1}}} \Delta^{\left(\frac{n}{2}-(\sigma-2)-3-\kappa_{1}\right)} \nabla^{i l} R_{i j k l} \otimes \nabla^{t_{1} \ldots t_{\kappa_{1}} j k} \psi_{1} \otimes \psi_{2} \otimes \Delta \psi_{3} \otimes \cdots \otimes \Delta \psi_{s-2}\right) .
$$

We then make two claims. Firstly, that modulo complete contractions of length $\geq \sigma+1$ :

$$
\operatorname{silly}_{*}\left[I_{g}^{s}\left(\psi_{1}, \ldots, \psi_{s}\right)\right]=0,
$$

and secondly that:

$$
\operatorname{silly}_{*}\left[I_{g}^{s}\left(\psi_{1}, \ldots, \psi_{s}\right)\right]=2^{\kappa_{1}} \frac{n-3}{n-2}(\text { const })_{\kappa_{1}} \cdot C_{g}^{\kappa_{1}}\left(\psi_{1}, \ldots, \psi_{s}\right),
$$

where $C_{g}^{\kappa_{1}}$ is in the form (3.62). Since $C^{\kappa_{1}}$ is clearly not identically zero, we will then deduce that (const) $\kappa_{\kappa_{1}}=0$, and we will have shown our inductive claim.

We begin with the first claim. We initially denote by silly ${ }_{+}\left[I_{g}^{s}\right]$ the sublinear combination in silly $\left[I_{g}^{s}\right]$ that consists of complete contractions with length $\sigma$ and a factor $\psi_{2}$, factors $\Delta \psi_{3}, \ldots, \Delta \psi_{s-2}$, a factor $\nabla^{\left(\kappa_{1}+2\right)} \psi_{1}$ with no internal contractions and a factor $\nabla^{(m)} R_{i j k l}$ (where now we are allowing any two indices in this last factor to be contracting against each other). In other words, we are looking at the sublinear combination of complete contractions in the form:

$$
\operatorname{contr}\left(\nabla^{(m)} R_{i j k l} \otimes \nabla^{\left(\kappa_{1}+2\right)} \psi_{1} \otimes \psi_{2} \otimes \Delta \psi_{3} \otimes \cdots \otimes \Delta \psi_{s}\right),
$$

where $\nabla^{\left(\kappa_{1}+2\right)} \psi_{1}$ has no internal contraction.

Since the silly divergence formula holds formally, we deduce that:

$$
\text { silly }{ }_{+}\left[I_{g}^{s}\right]=0,
$$

modulo complete contractions of length $\geq \sigma+1$.

We are now in a position to show our first claim above. By just applying the decomposition of the Weyl tensor, we calculate:

$$
\text { silly }_{+}\left[I_{g}^{s}\left(\psi_{1}, \ldots, \psi_{s}\right)\right]=\operatorname{silly}_{*}\left[I_{g}^{s}\left(\psi_{1}, \ldots, \psi_{s}\right)\right]+(\text { const })^{\prime} \cdot C_{g}^{\prime}\left(\psi_{1}, \ldots, \psi_{s}\right) \text {, }
$$

where $C_{g}^{\prime}\left(\psi_{1}, \ldots, \psi_{s}\right)$ is the complete contraction:

$$
\operatorname{contr}\left(\nabla^{t_{1} \ldots t_{\kappa_{1}+2}} \Delta^{\text {(power) }} R \otimes \nabla_{t_{1} \ldots t_{\kappa_{1}+2}} \psi_{1} \otimes \psi_{2} \otimes \Delta \psi_{3} \otimes \cdots \otimes \Delta \psi_{s}\right) .
$$

Now since silly $+\left[I_{g}^{s}\left(\psi_{1}, \ldots, \psi_{s}\right)\right]=0$, we may derive that the second sublinear combination in (3.64) vanishes separately ${ }^{39}$. Hence we derive our first claim, that silly ${ }_{*}\left[I_{g}^{s}\left(\psi_{1}, \ldots, \psi_{s}\right)\right]=0$.

We now proceed to the second claim. We would like to understand how the sublinear combination silly ${ }_{+}\left[I_{g}^{s}\right]$ is related to $I_{g}^{s}\left(\psi_{1}, \ldots, \psi_{s}\right)$. Given that $\mu=\frac{n}{2}-1$ in our case, while silly $+\left[I_{g}^{s}\right]$ consists of complete contractions with factors $\Delta \psi_{3}, \ldots, \Delta \psi_{s-1}$, it follows that a complete contraction $C_{g}\left(\psi_{1}, \ldots, \psi_{s-1}\right)$ in $I_{g}^{s}$ (where $C_{g}$ is in the form (3.44)) can only contribute to silly ${ }_{+}\left[I_{g}^{s}\right]$ if it has factors $\Delta \psi_{3}, \ldots, \Delta \psi_{s-1}$. (Otherwise, it must have at least one factor $\Delta^{y} \psi_{v}, v \geq 3$ with $y \geq 2$ ). Moreover, since the complete contractions in silly ${ }_{+}\left[I_{g}^{s}\right]$ each have a factor $\nabla^{\left(\kappa_{1}+2\right)} \psi_{1}$ with

\footnotetext{
${ }^{39}$ Using the operation $\mathrm{Sub}_{\omega}$, defined in the section on technical tools, in the Appendix of [3].
} 
no internal contractions, it follows that a complete contraction $C_{g}\left(\psi_{1}, \ldots, \psi_{s-1}\right)$ in $I_{g}^{s}$ (where $C_{g}$ is in the form (3.44)) can only contribute to silly ${ }_{+}\left[I_{g}^{s}\right]$ if it has a factor $\nabla^{(y)} \psi_{1}$ with no internal contractions (in the form (3.44)). Moreover, since we are considering complete contractions in $I_{g}^{s}\left(\psi_{1}, \ldots, \psi_{s}\right)$ with $\delta=\frac{n}{2}-1$, it follows that $y=2$, and therefore the two indices ${ }_{a},{ }_{b}$ in the factor $\nabla_{a b}^{(2)} \psi_{1}$ must be contracting against two internal indices in the one factor $\nabla^{(m)} W_{i j k l}$ (otherwise, if they contracted against at least one derivative index, we must have at least one pair of antisymmetric indices $_{i},{ }_{j}$ or ${ }_{k}, l$ in $\nabla^{(m)} W_{i j k l}$ both involved in an internal contraction, but such a complete contraction must clearly be zero modulo complete contractions of length $\geq \sigma+1$ ).

Therefore, we have that the complete contractions in $I_{g}^{s}\left(\psi_{1}, \ldots, \psi_{s}\right)$ that contribute to silly ${ }_{+}\left[I_{g}^{s}\left(\psi_{1}, \ldots, \psi_{s}\right)\right]$ must be in the form:

$$
\operatorname{contr}\left(\Delta^{(\alpha)} \nabla^{i l} W_{i j k l} \otimes \nabla^{j k} \psi_{1} \otimes \Delta^{(\beta)} \psi_{2} \otimes \Delta \psi_{3} \otimes \cdots \otimes \Delta \psi_{s}\right) .
$$

We denote the above complete contraction by $C_{g}^{\alpha, \beta}\left(\psi_{1}, \ldots, \psi_{s}\right)$. We recall that by our inductive assumption, each $C_{g}^{\alpha, \beta}$ in $I_{g}^{s}$ must have $\beta \leq \kappa_{1}$.

Clearly, we also observe that for each $C_{g}^{\alpha, \beta}$ above, the contribution of $C_{g}^{\alpha, \beta}$ to silly $\left[I_{g}^{s}\right]$ can only be in silly $\left[C_{g}^{\alpha, \beta}\right]$ if we integrate by parts all the $2 \beta$ indices $\left({ }^{a_{1}}, a_{1}\right), \ldots,\left({ }^{a_{\beta}}, a_{\beta}\right)$ in the factor $\Delta^{(\beta)} \psi_{2}$ and we make the derivatives $\nabla^{a_{1}}, \ldots, \nabla^{a_{\beta}}$ hit the factor $\nabla^{(m)} R_{i j k l}$ and the derivatives $\nabla_{a_{1}}, \ldots, \nabla_{a_{\beta}}$ hit the factor $\nabla^{(2)} \psi_{1}$. We observe that otherwise, we will either have an internal contraction in the factor $\nabla^{(p)} \psi_{1}$, or a factor $\nabla^{(p)} \psi_{h}, p, h \geq 3$, or a factor $\nabla^{(t)} \psi_{2}, t \geq 1$, or a factor $\nabla^{(y)} \psi_{1}$ with an internal contraction.

We denote the complete contraction that we thus obtain by $\hat{C}_{g}^{\alpha, \beta}$. Moreover, we clearly observe that unless $\beta=\kappa_{1}, \hat{C}_{g}^{\alpha, \beta}$ does not belong to silly ${ }_{+}\left[I_{g}^{s}\right]$. To summarize, we have seen that silly ${ }_{+}\left[I_{g}^{s}\right]$ is the sublinear combination in $\sum_{\gamma \in \Gamma_{\kappa_{1}}} a_{\gamma} C_{g}^{\gamma}\left(\psi_{1}, \ldots, \psi_{s}\right)$ that consists of complete contractions in the form (3.63).

We will now demonstrate our second claim. We employ the decomposition:

$$
\nabla^{i l} W_{i j k l}=\frac{n-3}{n-2} \nabla^{i l} R_{i j k l}+(\text { const })_{1} \nabla_{j k}^{2} R+(\text { const })_{2} \Delta R g_{j k} .
$$

We deduce that:

$$
\operatorname{silly}_{*}\left[I_{g}^{s}\left(\psi_{1}, \ldots, \psi_{s}\right)\right]=\sum_{\gamma \in \Gamma_{\kappa_{1}}} a_{\gamma} \frac{n-3}{n-2} C_{g}^{\gamma, \iota}\left(\psi_{1}, \ldots, \psi_{s}\right) .
$$

This shows our second claim.

\section{Acknowledgements}

This work has absorbed the best part of the author's energy over many years. This research was partially conducted during the period the author served as a Clay Research Fellow, an MSRI postdoctoral fellow, a Clay Liftoff fellow and a Procter Fellow. The author is immensely indebted to Charles Fefferman for devoting twelve long months to the meticulous proof-reading of the present paper. He also wishes to express his gratitude to the Mathematics Department of Princeton University for its support during his work on this project. 


\section{References}

[1] Alexakis S., On the decomposition of global conformal invariants. I, Ann. of Math. (2) 170 (2009), 12411306, arXiv:0711.1685.

[2] Alexakis S., On the decomposition of global conformal invariants. II, Adv. Math. 206 (2006), 466-502, arXiv:0912.3755.

[3] Alexakis S., The decomposition of global conformal invariants: a conjecture of Deser and Schwimmer, submitted.

[4] Alexakis S., The decomposition of global conformal invariants. IV. A proposition on local Riemannian invariants, arXiv:0912.3761.

[5] Alexakis S., The decomposition of global conformal invariants. V, arXiv:0912.3764.

[6] Atiyah M., Bott R., Patodi V.K., On the heat equation and the index theorem, Invent. Math. 19 (1973), $279-330$.

[7] Bailey T.N., Eastwood M.G., Gover A.R., Thomas's structure bundle for conformal, projective and related structures, Rocky Mountain J. Math. 24 (1994), 1191-1217.

[8] Bailey T.N., Eastwood M.G., Graham C.R., Invariant theory for conformal and CR geometry, Ann. of Math. (2) 139 (1994), 491-552.

[9] Berline N., Getzler E., Vergne M., Heat kernels and Dirac operators, Grundlehren Text Editions, SpringerVerlag, Berlin, 2004.

[10] Boulanger N., Algebraic classification of Weyl anomalies in arbitrary dimensions, Phys. Rev. Lett. 98 (2007), 261302, 4 pages, arXiv:0706.0340.

[11] Branson T., Gilkey P., Pohjanpelto J., Invariants of locally conformally flat manifolds, Trans. Amer. Math. Soc. 347 (1995), 939-953.

[12] Čap A., Gover A.R., Tractor calculi for parabolic geometries, Trans. Amer. Math. Soc. 354 (2002), 15111548.

[13] Čap A., Gover A.R., Standard tractors and the conformal ambient metric construction, Ann. Global Anal. Geom. 24 (2003), 231-259, math.DG/0207016.

[14] Cartan É., Sur la réduction à sa forme canonique de la structure d'un groupe de transformations fini et continu, Amer. J. Math. 18 (1896), 1-61.

[15] Deser S., Schwimmer A., Geometric classification of conformal anomalies in arbitrary dimensions, Phys. Lett. B 309 (1993), 279-284, hep-th/9302047.

[16] Fefferman C., Monge-Ampère equations, the Bergman kernel and geometry of pseudo-convex domains, Ann. of Math. (2) 103 (1976), 395-416, Erratum, Ann. of Math. (2), 104 (1976), 393-394.

[17] Fefferman C., Graham C.R., Conformal invariants, in The Mathematical Heritage of Élie Cartan (Lyon, 1984), Asterisque 1985 (1985), Numero Hors Serie, 95-116.

[18] Fefferman C., Graham C.R., The ambient metric, arXiv:0710.0919.

[19] Gilkey P.B., Local invariants of an embedded Riemannian manifold, Ann. of Math. (2) 102 (1975), $187-203$.

[20] Graham C.R., Extended obstruction tensors and renormalized volume coefficients, Adv. Math. 220 (2009), 1956-1985, arXiv:0810.4203.

[21] Graham C.R., Volume and area renormalizations for conformally compact Einstein metrics, in The Proceedings of the 19th Winter School "Geometry and Physics" (Srní, 1999), Rend. Circ. Mat. Palermo (2) Suppl. (2000), no. 63, 31-42, math.DG/9909042.

[22] Graham C.R., Hirachi K., Inhomogeneous ambient metrics, in Symmetries and Overdetermined Systems of Partial Differential Equations, IMA Vol. Math. Appl., Vol. 144, Springer, New York, 2008, 403-420, math.DG/0611931.

[23] Henningson M., Skenderis K., The holographic Weyl anomaly, J. High Energy Phys. 1998 (1998), no. 7, 023, 12 pages, hep-th/9806087.

[24] Hirachi K., Construction of boundary invariants and the logarithmic singularity of the Bergman kernel, Ann. of Math. (2) 151 (2000), 151-191, math.CV/0010014.

[25] Thomas T.Y., The differential invariants of generalized spaces, Cambridge University Press, Cambridge, 1934.

[26] Weyl H., The classical groups. Their invariants and representations, Princeton University Press, Princeton, 1997. 